\title{
REPRESENTATIONS OF THE EXCEPTIONAL LIE SUPERALGEBRA $E(3,6)$ II: FOUR SERIES OF DEGENERATE MODULES.
}

\author{
VICTOR G. KAC* AND ALEXEI RUDAKOV ${ }^{\dagger}$
}

\begin{abstract}
Four $\mathbb{Z}_{+}$-bigraded complexes with the action of the exceptional infinitedimensional Lie superalgebra $E(3,6)$ are constructed. We show that all the images and cokernels and all but three kernels of the differentials are irreducible $E(3,6)$-modules. This is based on the list of singular vectors and the calculation of homology of these complexes. As a result, we obtain an explicit construction of all degenerate irreducible $E(3,6)$-modules and compute their characters and sizes. Since the group of symmetries of the Standard Model $S U(3) \times S U(2) \times U(1)$ (divided by a central subgroup of order six) is a maximal compact subgroup of the group of automorphisms of $E(3,6)$, our results may have applications to particle physics.
\end{abstract}

\section{0 . Introduction.}

It has been established by A. Rudakov [R] some 25 years ago that all degenerate irreducible continuous modules over the Lie algebra $W_{n}$ of all formal vector fields in $n$ indeterminates occur as kernels and as cokernels of the differential of the $\mathbb{Z}_{+}$-graded de Rham complex $\Omega_{n}$ of all formal differential forms in $n$ indeterminates.

The main objective of the present paper is to obtain a similar result for the exceptional infinite-dimensional Lie superalgebra $E(3,6)$ from the list of simple linearly compact Lie superalgebras classified by V. Kac [K]. It turned out that the situation is much more interesting: we have constructed four $\mathbb{Z}_{+}$-bigraded complexes with the action of $E(3,6)$ and certain connecting homomorphisms between these complexes. All images and cokernels and all but three kernels of differentials turn out to be irreducible, and all degenerate irreducible $E(3,6)$-modules occur among them. The failure of irreducibility is connected to non-triviality of homology of these complexes, which we compute as well. At the end of the paper we compute the characters and sizes of all degenerate irreducible $E(3,6)$-modules and speculate on their relation to the Standard Model.

In our two other papers KKR1 and KR2 on the subject we show that all locally finite with respect to any non-trivial open subalgebra irreducible $E(3,6)$-modules, that do not occur as subquotients in our four complexes, are non-degenerate (and therefore induced).

\section{A reminder on $E(3,6)$ and induced modules.}

Recall that we view $E(3,6)$ as a subalgebra of the exceptional Lie superalgebra $E(5,10)$. The latter is constructed as follows (see $[\overline{\mathrm{CK}}]$ for details). The even part, $E(5,10)_{\overline{0}}$, is isomorphic to the Lie algebra $S_{5}$ of divergenceless formal vector fields in the indeterminates $x_{1}, \ldots, x_{5}$, while the odd part, $E(5,10)_{\overline{1}}$, is the space $d \Omega_{5}^{1}$ of closed (三 exact) 2 -forms, and

\footnotetext{
* Supported in part by NSF grant DMS-9970007.

$\dagger$ Research was partially conducted by Alexei Rudakov for the Clay Mathematics Institute.
} 
the remaining brackets are defined by:

$$
[X, w]=L_{X} w,\left[w, w^{\prime}\right]=w \wedge w^{\prime}, X \in S_{5}, w, w^{\prime} \in d \Omega_{5}^{1} .
$$

In the second formula a closed 4 -form $w \wedge w^{\prime}$ is identified with the vector field whose contraction with $d x_{1} \wedge \cdots \wedge d x_{5}$ produces this 4 -form. As in [KR1], we use the notation:

$$
d_{j k}=d x_{j} \wedge d x_{k}, \quad \partial_{i}=\partial / \partial x_{i} .
$$

Elements from $E(5,10)_{\overline{0}}$ are of the form

$$
\sum_{i} a_{i} \partial_{i}, \text { where } a_{i} \in \mathbb{C}\left[\left[x_{1}, \ldots, x_{5}\right]\right], \sum_{i} \partial_{i} a_{i}=0,
$$

and elements from $E(5,10)_{\overline{1}}$ are of the form

$$
w=\sum_{j, k} b_{j k} d_{j k} \text { where } b_{j k} \in \mathbb{C}\left[\left[x_{1}, \ldots, x_{5}\right]\right], d w=0 .
$$

In particular, the commutator of two odd elements can be computed using bilinearity and the rule $\left(a, b \in \mathbb{C}\left[\left[x_{1}, \ldots, x_{5}\right]\right]\right)$

$$
\left[a d_{j k}, b d_{\ell m}\right]=\epsilon_{i j k \ell m} a b \partial_{i},
$$

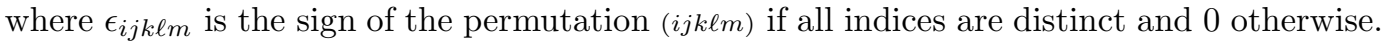
by

The Lie superalgebra $E(5,10)$ carries a unique consistent irreducible $\mathbb{Z}$-grading defined

$$
\operatorname{deg} x_{i}=2=-\operatorname{deg} \partial_{i}, \operatorname{deg} d x_{i}=-\frac{1}{2} .
$$

In order to define $E(3,6)$ as a subalgebra of $E(5,10)$, let $z_{+}=x_{4}, z_{-}=x_{5}, \partial_{+}=\partial_{4}$, $\partial_{-}=\partial_{5}$ and define the secondary $\mathbb{Z}$-grading by:

$$
\operatorname{deg} x_{i}=0=\operatorname{deg} \partial_{i}, \operatorname{deg} z_{ \pm}=1=-\operatorname{deg} \partial_{ \pm}, \operatorname{deg} d=-\frac{1}{2} .
$$

Then $E(3,6)$ is the $0^{\text {th }}$ piece of the secondary grading. The consistent $\mathbb{Z}$-grading of $E(5,10)$ induces the consistent $\mathbb{Z}$-grading $E(3,6)=L=\oplus_{j \geq-2} \mathrm{~g}_{j}$, where

$$
\mathrm{g}_{-2}=\left\langle\partial_{i} \mid i=1,2,3\right\rangle, \mathrm{g}_{-1}=\left\langle d_{i}^{+}:=d_{i 4}, d_{i}^{-}:=d_{i 5} \mid i=1,2,3\right\rangle .
$$

Furthermore, $\mathrm{g}_{0} \simeq s \ell(3) \oplus s \ell(2) \oplus g \ell(1)$, where

$$
\begin{aligned}
s \ell(3)= & \left\langle h_{1}=x_{1} \partial_{1}-x_{2} \partial_{2}, h_{2}=x_{2} \partial_{2}-x_{3} \partial_{3}, e_{1}=x_{1} \partial_{2},\right. \\
& \left.e_{2}=x_{2} \partial_{3}, e_{12}=x_{1} \partial_{3}, f_{1}=x_{2} \partial_{1}, f_{2}=x_{3} \partial_{2}, f_{12}=x_{3} \partial_{1}\right\rangle, \\
s \ell(2)= & \left\langle h_{3}=z_{+} \partial_{+}-z_{-} \partial_{-}, e_{3}=z_{+} \partial_{-}, f_{3}=z_{-} \partial_{+}\right\rangle, \\
g \ell(1)= & \left\langle Y=\frac{2}{3} \sum x_{i} \partial_{i}-\left(z_{+} \partial_{+}+z_{-} \partial_{-}\right)\right\rangle .
\end{aligned}
$$

The eigenspace decomposition of ad $(3 Y)$ coincides with the consistent $\mathbb{Z}$-grading of $E(3,6)$.

Below we often mark the elements $\partial_{i} \in \mathrm{g}_{-2}$ by a hat, writing $\hat{\partial}_{i}$ whenever we need to distinguish them from $\partial_{i}$ in another role.

As has been mentioned in [CK] and [KR1], the even part $E(3,6)_{\overline{0}}$ of $E(3,6)$ contains a subalgebra $W$ isomorphic to $W_{3}$ with the isomorphism given by the formula

$$
D \longmapsto D-\frac{1}{2} \operatorname{div} D\left(z_{+} \partial_{+}+z_{-} \partial_{-}\right) .
$$

We fix the Cartan subalgebra $\mathcal{H}=\left\langle h_{1}, h_{2}, h_{3}, Y\right\rangle$ and the Borel subalgebra $\mathcal{B}=\mathcal{H}+\left\langle e_{i}(i=1,2,3), e_{12}\right\rangle$ of $\mathrm{g}_{0}$. Then $f_{0}:=d_{1}^{+}$is the highest weight vector of the (irreducible) $\mathrm{g}_{0}$-module $\mathrm{g}_{-1}$,

$$
e_{0}^{\prime}:=x_{3} d_{3}^{-} \text {and } e_{0}:=x_{3} d_{2}^{-}-x_{2} d_{3}^{-}+2 x_{5} d_{23}
$$


are the lowest weight vectors of the $\mathrm{g}_{0}$-module $\mathrm{g}_{1}$, and one has:

so that

$$
\begin{aligned}
& {\left[e_{0}^{\prime}, f_{0}\right]=f_{2},} \\
& {\left[e_{0}, f_{0}\right]=\frac{2}{3} h_{1}+\frac{1}{3} h_{2}-h_{3}-Y=: h_{0},}
\end{aligned}
$$

The following relations are also important to keep in mind:

$$
\begin{aligned}
{\left[e_{0}^{\prime}, d_{1}^{+}\right] } & =f_{2},\left[e_{0}^{\prime}, d_{2}^{+}\right]=-f_{12},\left[e_{0}^{\prime}, d_{3}^{+}\right]=0,\left[e_{0}^{\prime}, d_{i}^{-}\right]=0, \\
{\left[d_{i}^{ \pm}, d_{j}^{ \pm}\right] } & =0, \quad\left[d_{i}^{+}, d_{j}^{-}\right]+\left[d_{j}^{+}, d_{i}^{-}\right]=0 .
\end{aligned}
$$

Recall that $\mathrm{g}_{0}$ along with the elements $f_{0}, e_{0}, e_{0}^{\prime}$ generate the Lie superalgebra $E(3,6)$.

Sometimes we use the following shorthand notation for the elements of the universal enveloping algebra of $E(3,6)$ :

$$
d_{i j k}^{ \pm}=d_{i}^{ \pm} d_{j}^{ \pm} d_{k}^{ \pm} .
$$

We have the triangular decomposition:

$$
L:=E(3,6)=L_{-}+\mathrm{g}_{0}+L_{+},
$$

where $L_{-}=\oplus_{j<0} \mathrm{~g}_{j}, L_{+}=\prod_{j>0} \mathrm{~g}_{j}$. Given a $\mathrm{g}_{0}$-module $V$, we extend it to $L_{0}:=\mathrm{g}_{0}+L_{+}$ by letting $L_{+}$act trivially and consider the induced $L$-module

$$
M(V)=U(L) \otimes_{U\left(L_{0}\right)} V \simeq U\left(L_{-}\right) \otimes_{\mathbb{C}} V,
$$

the latter being a $\mathrm{g}_{0}$-isomorphism. If $V$ is a finite-dimensional irreducible $\mathrm{g}_{0}$-module, the $L$-module $M(V)$ is called a generalized Verma module. Any quotient of a generalized Verma module is called a highest weight module.

Recall that all finite-dimensional irreducible $\mathrm{g}_{0}$-modules are modules with highest weight $\lambda$ such that

$$
p=\lambda\left(h_{1}\right), q=\lambda\left(h_{2}\right), r=\lambda\left(h_{3}\right) \in \mathbb{Z}_{+}, y=\lambda(Y) \in \mathbb{C} .
$$

Such a module is denoted by $F(p, q ; r ; y)$ and the corresponding generalized Verma $L$-module is denoted by $M(p, q ; r ; y)$. A highest weight vector of the $\mathrm{g}_{0}$-module $F(p, q ; r ; y)$ is called a highest weight vector of any corresponding highest weight $L$-module.

Note that the hypercharge operator $Y$ acts diagonally on $M(p, q ; r ; y)$ with eigenvalues from the set $y-\frac{1}{3} \mathbb{Z}_{+}$, its eigenspaces are $\mathrm{g}_{0}$-invariant and finite-dimensional, and the $y$-eigenspace is isomorphic to the $\mathrm{g}_{0}$-module $F(p, q ; r ; y)$.

A highest weight vector $s$ of an irreducible finite-dimensional $\mathrm{g}_{0}$-submodule of an $L$-module is called singular if

$$
\left.e_{0}^{\prime} s=0, \quad e_{0} s=0 \quad \text { (equivalently: } L_{+} s=0\right) .
$$

For example, the highest weight vector of $F(p, q ; r, y) \subset M(p, q ; r ; y)$ is a singular vector, called a trivial singular vector.

Recall that the $L$-module $M(p, q ; r ; y)$ has a unique irreducible quotient denoted by $I(p, q ; r ; y)$. The irreducible $L$-module $I(p, q ; r ; y)$ is called non-degenerate if it coincides with $M(p, q ; r ; y)$, the module $I(p, q ; r ; y)$ and the module $M(p, q ; r ; y)$ are called degenerate if $M(p, q ; r ; y) \neq I(p, q ; r ; y)$.

It follows that $M(p, q, ; r ; y)$ is a degenerate $L$-module iff it has a non-trivial singular vector.

The main result of [KR1] is the following.

Theorem 1.1. If $p q \neq 0$, then the $E(3,6)$-module $I(p, q ; r ; y)$ is non-degenerate.

The corollary of the main result of [KR2] is the following. 
Theorem 1.2. The following list consists of all degenerate $E(3,6)$-modules $I(p, q ; r ; y)$ $\left(p, q, r \in \mathbb{Z}_{+}\right)$:

type $A: I\left(p, 0 ; r ; y_{A}\right)$, where $y_{A}=\frac{2}{3} p-r$,

type $B: \quad I\left(p, 0 ; r ; y_{B}\right)$, where $y_{B}=\frac{2}{3} p+r+2$,

type $C: \quad I\left(0, q ; r, y_{C}\right)$, where $y_{C}=-\frac{2}{3} q-r-2$,

type $D: \quad I\left(0, q ; r, y_{D}\right)$, where $y_{D}=-\frac{2}{3} q+r$.

In the present paper we construct four complexes of degenerate generalized Verma modules, which allows us to construct the four series of degenerate modules given by Theorem 1.2.

\section{The four bigraded complexes.}

Given a $\mathrm{g}_{0}$-module $V$ and a number $a \in \mathbb{C}$, we can define a new $\mathrm{g}_{0}$-module, denoted by $V_{[a]}$ with the same action of $s \ell(3) \oplus s \ell(2)$ but the changed action of $Y$ by adding to it $a I_{V}$. Consider the following $\mathrm{g}_{0}$-modules:

$$
\begin{aligned}
V_{A} & =\mathbb{C}\left[x_{1}, x_{2}, x_{3}, z_{+}, z_{-}\right], \\
V_{B} & =\mathbb{C}\left[x_{1}, x_{2}, x_{3}, \partial_{+}, \partial_{-}\right]_{[2]}, \\
V_{C} & =\mathbb{C}\left[\partial_{1}, \partial_{2}, \partial_{3}, z_{+}, z_{-}\right]_{[-2]}, \\
V_{D} & =\mathbb{C}\left[\partial_{1}, \partial_{2}, \partial_{3}, \partial_{+}, \partial_{-}\right],
\end{aligned}
$$

with the action of $\mathrm{g}_{0}$ given by (1.1)-(1.3). The decomposition into a sum of irreducible $\mathrm{g}_{0}$-modules is given by the bigrading $(X=A, B, C, D)$

$$
V_{X}=\bigoplus_{m, n \in \mathbb{Z}} V_{X}^{m, n}
$$

where

$$
V_{X}^{m, n}=\left\{f \in V_{X} \mid \sum_{i} x_{i} \partial_{i} f=m f, \sum_{\epsilon} z_{\epsilon} \partial_{\epsilon} f=n f\right\} .
$$

Let $M_{X}=M\left(V_{X}\right)$ be the corresponding induced $L$-module. The bigrading of $V_{X}$ gives rise to a bigrading of $M_{X}$ by generalized Verma modules:

$$
M_{X}=\bigoplus_{m, n \in \mathbb{Z}} M\left(V_{X}^{m ; n}\right) .
$$

Note that we have the following isomorphisms of $\mathrm{g}_{0}$-modules (see (1.1)):

$$
\begin{aligned}
V_{A}^{p, r} & \simeq F\left(p, 0 ; r ; y_{A}\right), \\
V_{B}^{p,-r} & \simeq F\left(p, 0 ; r ; y_{B}\right), \\
V_{C}^{-q, r} & \simeq F\left(0, q ; r ; y_{C}\right), \\
V_{D}^{-q,-r} & \simeq F\left(0, q ; r ; y_{D}\right) .
\end{aligned}
$$

Consequently, the bigrading (2.1) takes the form:

$$
\begin{aligned}
& M_{X}=\oplus_{p, r \in \mathbb{Z}_{+}} M\left(p, 0 ; r ; y_{X}\right), \text { for } X=A \text { or } B, \\
& M_{X}=\oplus_{q, r \in \mathbb{Z}_{+}} M\left(0, q ; r, y_{X}\right), \text { for } X=C \text { or } D .
\end{aligned}
$$

We let the algebra $U\left(L_{-}\right) \otimes$ End $V_{X}$ act on $M_{X}=U\left(L_{-}\right) \otimes V_{X}$ by the formula:

$$
(u \otimes \varphi)\left(u^{\prime} \otimes v\right)=u^{\prime} u \otimes \varphi(v), u, u^{\prime} \in U\left(L_{-}\right), \varphi \in \text { End } V_{X}, v \in V_{X} .
$$

We shall often drop the $\otimes$ sign, e.g. we shall write $\partial_{i}$ in place of $1 \otimes \partial_{i}$, etc. 
Introduce the following operators on $M_{X}$ (acting by (2.3)):

$$
\begin{aligned}
\Delta^{ \pm} & =\sum_{i=1}^{3} d_{i}^{ \pm} \otimes \partial_{i}, \\
\delta_{i} & =d_{i}^{+} \otimes \partial_{+}+d_{i}^{-} \otimes \partial_{-}, i=1,2,3 .
\end{aligned}
$$

Here $\partial_{i}$ acts as $d / d x_{i}$ (resp. as multiplication by $\partial_{i}$ ) in the cases $X=A, B($ resp. $X=C, D)$ and similarly for $\partial_{ \pm}$.

Lemma 2.1. (a) The action of $s \ell(3)$ (resp. $s \ell(2)) \subset \mathrm{g}_{0} \subset E(3,6)$ on the $E(3,6)$ module $M_{X}$ commutes with the operators $\Delta^{ \pm}$and $\partial_{ \pm}$(resp. $\delta_{i}$ and $\partial_{i}$ ).

(b) One has the following commutation relations for $Y$ :

$$
\left[Y, \partial_{ \pm}\right]=\partial_{ \pm},\left[Y, \partial_{i}\right]=-\frac{2}{3} \partial_{i},\left[Y, d_{i}^{ \pm}\right]=-\frac{1}{3} d_{i}^{ \pm},\left[Y, \Delta^{ \pm}\right]=-\Delta^{ \pm},\left[Y, \delta_{i}\right]=\frac{2}{3} \delta_{i} .
$$

(c) $\left(\Delta^{ \pm}\right)^{2}=0, \Delta^{+} \Delta^{-}+\Delta^{-} \Delta^{+}=0, \quad \delta_{i} \delta_{j}+\delta_{j} \delta_{i}=0$.

Proof. The proof of (a) and (b) is straightforward, (c) follows from (1.9).

Now we introduce our basic operator

$$
\nabla:=\Delta^{+} \partial_{+}+\Delta^{-} \partial_{-}=\delta_{1} \partial_{1}+\delta_{2} \partial_{2}+\delta_{3} \partial_{3} .
$$

Proposition 2.2. $\quad$ (a) $\nabla^{2}=0$.

(b) The operator $\nabla$ commutes with the action of $E(3,6)$ on $M_{X}$.

Proof. (a) is immediate by Lemma 2.1c. In order to prove (b), notice that the action (2.3) commutes with the left multiplication action of $L_{-}$on $M_{X}$, hence, in particular, $\nabla$ commutes with $L_{-}$. Furthermore, it follows from Lemma 2.1 a (resp. 2.1 b) that $\nabla$ commutes with the action of $s \ell(3)$ and $s \ell(2)$ (resp. $Y$ ), hence $\nabla$ commutes with $g_{0}$.

Now the proof that the operator $\nabla$ commutes with $L$ is based on the following lemma.

Lemma 2.3. Let $L=\prod_{j} \mathrm{~g}_{j}$ be a $\mathbb{Z}$-graded Lie superalgebra and let $M$ be an L-module induced from an $L_{0}$-module $V$ such that $\left.\mathrm{g}_{j}\right|_{V}=0$ for $j>0$. Let $\nabla$ be an operator acting on $M$ that commutes with $L_{-}$. Suppose that $\nabla$ commutes with $\mathrm{g}_{0}$ and

$$
g \nabla(v)=0 \text { for all } g \in L_{+}, v \in 1 \otimes V .
$$

Then $\nabla$ commutes with $L$.

Proof. It is sufficient to show that $\nabla$ commutes with $L_{+}$. For $g \in L_{+}, u \in L_{-}$we can write:

$$
g u=\sum_{n \geq 0} u_{n} g_{n}, \text { where } u_{n} \in U\left(L_{-}\right), g_{n} \in \mathrm{g}_{n} .
$$

Then we have:

$$
\begin{aligned}
g \nabla(u \otimes v) & =g u \nabla(v)=\sum_{n \geq 0} u_{n} g_{n} \nabla(v) \\
& =u_{0} g_{0} \nabla(v)=u_{0} \nabla g_{0}(v)=\sum_{n \geq 0} u_{n} \nabla g_{n}(v)=\nabla g(u \otimes v) .
\end{aligned}
$$


We shall establish that the conditions of Lemma 2.3 are valid in our situation. Since $\left\{g \in L_{+} \mid g \nabla(v)=0\right.$ for all $\left.v \in 1 \otimes V_{X}\right\}$ is a $g_{0}$-invariant subalgebra of $L_{+}$(because $\nabla$ commutes with $\mathrm{g}_{0}$ ), in order to establish (2.6), it suffices to check only the following:

$$
\begin{aligned}
& e_{0}^{\prime} \nabla(v)=0, v \in 1 \otimes V_{X}, \\
& e_{0} \nabla(v)=0, v \in 1 \otimes V_{X} .
\end{aligned}
$$

(Recall that $\mathrm{g}_{1}$ generates $L_{+}$and $e_{0}, e_{0}^{\prime}$ generate the $\mathrm{g}_{0}$-module $\mathrm{g}_{1}$.) Using (1.8), we get 2.7):

$$
e_{0}^{\prime} \nabla(v)=\sum_{\epsilon=+,-}\left(f_{2} \partial_{1} \partial_{\epsilon} v-f_{12} \partial_{2} \partial_{\epsilon} v\right)=\left(\left(x_{3} \partial_{2}\right) \partial_{1}-\left(x_{3} \partial_{1}\right) \partial_{2}\right) \sum_{\epsilon} \partial_{\epsilon} v=0 .
$$

Furthermore, we have:

$$
e_{0} \nabla(v)=h_{0}\left(\partial_{1} \partial_{+} v\right)+f_{1}\left(\partial_{2} \partial_{+} v\right)+f_{12}\left(\partial_{3} \partial_{+} v\right)-2 f_{3}\left(\partial_{1} \partial_{-} v\right) .
$$

In the $X=A$ case, $h_{0}$ acts on $V_{X}$ by (1.7) (where $x_{4}=z_{+}, x_{5}=z_{-}$), $f_{1}=x_{2} \partial_{1}$, $f_{12}=x_{3} \partial_{1}$ and $f_{3}=z_{-} \partial_{+}$, hence (2.9) becomes:

$$
e_{0} \nabla(v)=\left(h_{0}+x_{2} \partial_{2}+x_{3} \partial_{3}-2 z_{-} \partial_{-}\right) \partial_{1} \partial_{+} v=0 .
$$

In the $X=B$ case, $h_{0}$ acts on $V_{X}$ by "(1.7) minus $2 I$ " due to the twist for $Y$, which is necessary to include in order to compensate for the additional term $2 \partial_{1} \partial_{+} v$ occurring from the last term on the right of (2.9).

Similar calculations show that (2.8) holds in the $X=C$ and $D$ cases as well.

Remark 2.4. (a) Since $\nabla$ commutes with the representation of $E(3,6)$ in $M_{X}$, the nonzero image under $\nabla$ of a singular vector is a singular vector.

(b) Let $V$ be a direct sum of finite-dimensional irreducible $\mathrm{g}_{0}$-modules, $M(V)$ be the corresponding induced $E(3,6)$-module, and let $\nabla$ be an operator on $M(V)$ defined via (2.3), such that $\nabla v$ is a singular vector for each of the highest weight vectors $v$ of $\mathrm{g}_{0}$ in $V$. Suppose that $\nabla$ commutes with $Y$ and all $f_{i}, i=1,2,3$, then $\nabla$ commutes with $E(3,6)$. This follows from Lemma 2.3 .

(c) Suppose that for the same module $M(V)$ there is a linear map $\varphi: M(V) \rightarrow N$ to another $L$-module $N$. If $\varphi$ commutes with the action of $L$, then the non-zero image of a singular vector is a singular vector. If $\varphi$ commutes with the action of $L_{-}$, the image $\varphi(v)$ is a singular vector for each of the highest weight vectors $v$ of $\mathrm{g}_{0}$ in $V$, $\varphi$ commutes with $\mathrm{g}_{0}$, then $\varphi$ is a morphism of $L$-modules. The arguments are just the same as for (a), (b).

(d) Lemma 2.3 and the above remark (c) could be generalized to the case when $M$ is a highest weight $L$-module, or a sum of such modules.

Proposition 2.2 implies immediately the following corollary about singular vectors.

Corollary 2.5. (a) The $E(3,6)$-module $M\left(p, 0 ; r ; y_{A}\right)$ has a non-trivial singular vector

$$
\nabla\left(x_{1}^{p+1} z_{+}^{r+1}\right)=(p+1)(r+1) d_{1}^{+} x_{1}^{p} z_{+}^{r}
$$

for all $p, r \in \mathbb{Z}_{+}$.

(b) The $E(3,6)$-module $M\left(p, 0 ; r ; y_{B}\right)$ has a non-trivial singular vector

$$
\nabla\left(x_{1}^{p+1} \partial_{-}^{r-1}\right)=(p+1) \delta_{1} x_{1}^{p} \partial_{-}^{r-1}
$$

for all $p, r \in \mathbb{Z}_{+}, r>0$. 
(c) The $E(3,6)$-module $M\left(0, q ; r ; y_{C}\right)$ has a nontrivial singular vector

$$
\nabla\left(\partial_{3}^{q-1} z_{+}^{r+1}\right)=(r+1) \Delta^{+} \partial_{3}^{q-1} z_{+}^{r}
$$

for all $q, r \in \mathbb{Z}_{+}, q>0$.

(d) The $E(3,6)$-module $M\left(0, q ; r ; y_{D}\right)$ has a nontrivial singular vector

$$
\nabla\left(\partial_{3}^{q-1} \partial_{-}^{r-1}\right)
$$

for all $q, r \in \mathbb{Z}_{+}, q, r>0$.

In order to construct more singular vectors, consider smaller $g_{0}$-modules $V_{X^{\prime}} \subset V_{X}$ and the corresponding induced $L$-modules $M_{X^{\prime}} \subset M_{X}$ :

$$
\begin{array}{llll}
V_{A^{\prime}}=\mathbb{C}\left[x_{1}, x_{2}, x_{3}\right] & \text { and } & & M_{A^{\prime}}=M\left(V_{A^{\prime}}\right), \\
V_{B^{\prime}}=\mathbb{C}\left[x_{1}, x_{2}, x_{3}\right]_{[2]} & \text { and } & M_{B^{\prime}}=M\left(V_{B^{\prime}}\right), \\
V_{C^{\prime}}=\mathbb{C}\left[\partial_{1}, \partial_{2}, \partial_{3}\right]_{[-2]} & \text { and } & & M_{C^{\prime}}=M\left(V_{C^{\prime}}\right), \\
V_{D^{\prime}}=\mathbb{C}\left[\partial_{1}, \partial_{2}, \partial_{3}\right] & \text { and } & & M_{D^{\prime}}=M\left(V_{D^{\prime}}\right) .
\end{array}
$$

Let $\tau_{1}: V_{A^{\prime}} \underset{\rightarrow}{\rightarrow} V_{B^{\prime}}$ and $\tau_{2}: V_{C^{\prime}} \underset{\rightarrow}{\rightarrow} V_{D^{\prime}}$ be the identity isomorphisms. Then we have:

$$
\left[Y, \tau_{i}\right]=2 \tau_{i} .
$$

Let

$$
\begin{aligned}
& \nabla_{2}=\Delta^{+} \Delta^{-} \tau_{1}: M_{A^{\prime}} \rightarrow M_{B^{\prime}}, \\
& \nabla_{2}=\Delta^{+} \Delta^{-} \tau_{2}: M_{C^{\prime}} \rightarrow M_{D^{\prime}}
\end{aligned}
$$

act via (2.3).

\section{Proposition 2.6. $\quad$ (a) $\nabla \nabla_{2}=\nabla_{2} \nabla=0$.}

(b) $\nabla_{2}$ is an $E(3,6)$-module isomorphism: $M_{X^{\prime}} \stackrel{\sim}{\rightarrow} M_{Y^{\prime}}$, where $X^{\prime}=A^{\prime}$ (resp. $C^{\prime}$ ) and $Y^{\prime}=B^{\prime}\left(\right.$ resp., $\left.D^{\prime}\right)$.

Proof. (a) is clear from Lemma 2.1c. Next, $L_{-}$commutes with $\nabla_{2}$ by definition, $s \ell(3)$ commutes due to Lemma 2.1, $s \ell(2)$ commutes for trivial reasons and $Y$ commutes due to Lemma 2.1 b and 2.10).

As before, due to Lemma 2.3, in order to establish (b), it suffices to show that

$$
e_{0}^{\prime} \nabla_{2} v=0 \text { and } e_{0} \nabla_{2} v=0 \text { for } v \in 1 \otimes V_{X^{\prime}} .
$$

Let $X^{\prime}=A^{\prime}$. We have:

$$
\begin{aligned}
e_{0}^{\prime} \nabla_{2} v & =e_{0}^{\prime}\left(-\Delta^{-} \Delta^{+} \tau_{1}\right)(v) \\
& =\Delta^{-}\left(-f_{2} \partial_{1}+f_{12} \partial_{2}\right) \tau_{1}(v)=\Delta^{-}\left(-\left(x_{3} \partial_{2}\right) \partial_{1}+\left(x_{3} \partial_{1}\right) \partial_{2}\right) \tau_{1}(v)=0 .
\end{aligned}
$$

Next, we have:

$$
\begin{aligned}
e_{0} \nabla_{2} v & =\left(\left(2 f_{3} \partial_{1}\right) \Delta^{+}+\Delta^{-}\left(h_{0} \partial_{1}+f_{1} \partial_{2}+f_{12} \partial_{3}\right)\right) \tau_{i}(v) \\
& =\Delta^{-}\left(\left(2+h_{0}\right) \partial_{1}+\left(x_{2} \partial_{1}\right) \partial_{2}+\left(x_{3} \partial_{1}\right) \partial_{3}\right) \tau_{i}(v)
\end{aligned}
$$

because $f_{3} \tau_{i}(v)=0$, since $f_{3}$ annihilates $B^{\prime}$ and $D^{\prime}$. Notice that for $v=x_{1}^{a_{1}} x_{2}^{a_{2}} x_{3}^{a_{3}}$ :

$$
\left(x_{j} \partial_{1}\right) \partial_{j} \tau_{1}(v)=\left(x_{j} \partial_{j}\right) \partial_{1} \tau_{1}(v), \quad j=2,3,
$$

but for $v=\partial_{1}^{a_{1}} \partial_{2}^{a_{1}} \partial_{3}^{a_{1}}$ :

$$
\left(x_{j} \partial_{1}\right) \partial_{j} \tau_{2}(v)=\left(x_{j} \partial_{j}-1\right) \partial_{1} \tau_{2}(v), \quad j=2,3 .
$$


At the same time $\left.h_{0}\right|_{B^{\prime}}=-x_{2} \partial_{2}-x_{3} \partial_{3}+2 z_{-} \partial_{-}-2$ and $\left.h_{0}\right|_{D^{\prime}}=-x_{2} \partial_{2}-x_{3} \partial_{3}+2 z_{-} \partial_{-}$. Therefore

$$
\begin{aligned}
e_{0} \nabla_{2} v & =\Delta^{-}\left(\left(2+h_{0}\right) \partial_{1}+\left(x_{2} \partial_{1}\right) \partial_{2}+\left(x_{3} \partial_{1}\right) \partial_{3}\right) \tau_{i}(v), \\
& =\Delta^{-}\left(2 z_{-} \partial_{-}\right) \tau_{i}(v)=0 .
\end{aligned}
$$

Finally, in a similar fashion, consider the $\mathrm{g}_{0}$-modules $V_{X^{\prime \prime}} \subset V_{X}$ and the corresponding induced $L$-modules $M_{X^{\prime \prime}} \subset M_{X}$ :

$$
\begin{array}{rlrl}
V_{A^{\prime \prime}}=\mathbb{C}\left[z_{+}, z_{-}\right] & \text {and } & & M_{A^{\prime \prime}}=M\left(V_{A^{\prime \prime}}\right), \\
V_{B^{\prime \prime}}=\mathbb{C}\left[\partial_{+}, \partial_{-}\right]_{[2]} & \text { and } & & M_{B^{\prime \prime}}=M\left(V_{B^{\prime \prime}}\right), \\
V_{C^{\prime \prime}}=\mathbb{C}\left[z_{+}, z_{-}\right]_{[-2]} & \text { and } & & M_{C^{\prime \prime}}=M\left(V_{C^{\prime \prime}}\right), \\
V_{D^{\prime \prime}}=\mathbb{C}\left[\partial_{+}, \partial_{-}\right] & \text {and } & M_{D^{\prime \prime}}=M\left(V_{D^{\prime \prime}}\right) .
\end{array}
$$

We let $\rho_{1}: V_{A^{\prime \prime}} \stackrel{\sim}{\rightarrow} V_{C^{\prime \prime}}$ and $\rho_{2}: V_{B^{\prime \prime}} \underset{\rightarrow}{\rightarrow} V_{D^{\prime \prime}}$ be the identity isomorphisms, so that

$$
\left[Y, \rho_{i}\right]=-2 \rho_{i} .
$$

Let

$$
\begin{aligned}
& \nabla_{3}=\delta_{1} \delta_{2} \delta_{3} \rho_{1}: M_{A^{\prime \prime}} \rightarrow M_{C^{\prime \prime}}, \\
& \nabla_{3}=\delta_{1} \delta_{2} \delta_{3} \rho_{2}: M_{B^{\prime \prime}} \rightarrow M_{D^{\prime \prime}}
\end{aligned}
$$

act via (2.3).

Proposition 2.7. $\quad$ (a) $\nabla \nabla_{3}=\nabla_{3} \nabla=0$.

(b) $\nabla_{3}$ is an $E(3,6)$-module isomorphism $M_{X^{\prime \prime}} \rightarrow M_{Y^{\prime \prime}}$, where $X^{\prime \prime}=A^{\prime \prime}$ (resp. $B^{\prime \prime}$ ) and $Y^{\prime \prime}=C^{\prime \prime}\left(\right.$ resp. $\left.D^{\prime \prime}\right)$.

Proof. As in the proof of Proposition 2.6, due to Lemma 2.1, (a) is clear and (b) reduces to checking the relations

$$
e_{0}^{\prime} \nabla_{3} v=0 \text { and } e_{0} \nabla_{3} v=0 \text { for } v \in 1 \otimes V_{X} .
$$

We have

$$
e_{0}^{\prime} \nabla_{3} v=\left(f_{2} \partial_{+} \delta_{2} \delta_{3}+\delta_{1} f_{12} \partial_{+} \delta_{3}\right) \rho_{1} v=\partial_{+} \delta_{3}^{2} \rho_{1} v=0
$$

if $X^{\prime \prime}=A^{\prime \prime}$ because $f_{2} \rho_{1} v=f_{12} \rho_{1} v=0$, and similarly for $X^{\prime \prime}=B^{\prime \prime}$.

Next, we have for $v \in V_{X^{\prime \prime}}$ :

$$
\begin{aligned}
{\left[e_{0}, \delta_{1} \delta_{2} \delta_{3}\right] v } & =\left(\left(h_{0} \partial_{+}-2 f_{3} \partial_{-}\right) \delta_{2} \delta_{3}-\delta_{1} f_{1} \partial_{+} \delta_{3}+\delta_{1} \delta_{2} f_{12} \partial_{+}\right) v \\
& =\left(h_{0} \partial_{+}-2 f_{3} \partial_{-}\right) \delta_{2} \delta_{3}(v) .
\end{aligned}
$$

Since $\left[h_{0}, \delta_{i}\right]=-\delta_{i}$ for $i=1,2$ and $\left[f_{3}, \delta_{i}\right]=0$, we obtain for $v \in V_{X^{\prime \prime}}$ :

$$
\left[e_{0}, \delta_{1} \delta_{2} \delta_{3}\right] v=\delta_{2} \delta_{3}\left(\left(h_{0}-2\right) \partial_{+} v-2\left(z_{-} \partial_{+}\right) \partial_{-} v\right) \text {. }
$$

Again $\left(z_{-} \partial_{+}\right) \partial_{-} v=\left(z_{-} \partial_{-}\right) \partial_{+} v$ for $v \in C^{\prime \prime}$ and $\left(z_{-} \partial_{+}\right) \partial_{-} v=\left(z_{-} \partial_{-}-1\right) \partial_{+} v$ for $v \in D^{\prime \prime}$.

In the case $X^{\prime \prime}=A^{\prime \prime}$ we have:

$$
e_{0} \delta_{1} \delta_{2} \delta_{3} \rho_{1} v=\delta_{2} \delta_{3}\left(h_{0}-2 z_{-} \partial_{+}-2\right) \partial_{-} \rho_{1} v=0 .
$$

In the case $X^{\prime \prime}=B^{\prime \prime}$, we similarly deduce:

$$
e_{0} \delta_{1} \delta_{2} \delta_{3} \rho_{2} v=\delta_{2} \delta_{3}\left(h_{0}-2 z_{-} \partial_{+}\right) \partial_{-} \rho_{2} v=0 .
$$

Propositions 2.6 and 2.7 lead to the next corollary about singular vectors. 
Corollary 2.8. (a) The $E(3,6)$-module $M\left(p, 0 ; 0 ; y_{B}\right)$ has a non-trivial singular vector

$$
\nabla_{2}\left(x_{1}^{p+2}\right)=(p+2)(p+1) d_{1}^{+} d_{1}^{-} x_{1}^{p}
$$

for all $p \in \mathbb{Z}_{+}$.

(b) The $E(3,6)$-module $M\left(0, q ; 0 ; y_{D}\right)$ has a non-trivial singular vector

$$
\nabla_{2}\left(\partial_{3}^{q-2}\right)=\Delta^{+} \Delta^{-} \partial_{3}^{q-2}
$$

for all $q \in \mathbb{Z}, q \geq 2$

(c) The $E(3,6)$-module $M\left(0,0 ; r ; y_{C}\right)$ has a non-trivial singular vector

$$
\nabla_{3}\left(z_{+}^{r+3}\right)=(r+3)(r+2)(r+1) d_{123}^{+} z_{+}^{r}
$$

for all $r \in \mathbb{Z}_{+}$.

(d) The $E(3,6)$-module $M\left(0,0 ; r ; y_{D}\right)$ has a non-trivial singular vector

$$
\nabla_{3}\left(\partial_{-}^{r-3}\right)
$$

for all $r \in \mathbb{Z}_{+}, r \geq 3$.

There are a few more singular vectors. One is the vector

$$
w_{1}=d_{123}^{+} \Delta^{-} 1
$$

of the $E(3,6)$-module $M\left(0,1 ; 0 ; y_{D}\right)$. It is straightforward to show by checking that $e_{0}^{\prime} w_{1}=0$ and $e_{i} w_{1}=0$ for $i=0, \ldots, 3$. This singular vector generates a non-zero homomorphism of $E(3,6)$-modules $\nabla_{4}^{\prime}: M\left(0,0 ; 2 ; y_{A}\right) \rightarrow M\left(0,1 ; 0 ; y_{D}\right)$ such that for the highest weight vector $z_{+}^{2}$ of $M\left(0,0 ; 2 ; y_{A}\right)$ we have

$$
\nabla_{4}^{\prime}\left(z_{+}^{2}\right)=d_{123}^{+} \Delta^{-} 1
$$

We shall be looking for $\nabla_{4}^{\prime}$ in the form:

$$
\nabla_{4}^{\prime}=a \Delta^{-} \partial_{+}^{2}+b \Delta^{-} \partial_{+} \partial_{-}+c \Delta^{-} \partial_{-}^{2}, \quad a=d_{1}^{+} d_{2}^{+} d_{3}^{+},
$$

where $b, c \in U\left(L_{-}\right)$. Here all three operators $\partial_{+}^{2}, \partial_{+} \partial_{-}$and $\partial_{-}^{2}$ map $V_{A}^{0 ; 2}$, which is the space of quadratic polynomials in $z_{ \pm}$, to $\mathbb{C}$, hence operator (2.14) may be viewed as a linear map from $M\left(0,0 ; 2 ; y_{A}\right)$ to $M\left(0,1 ; 0 ; y_{D}\right)$. It is clear that, applied to the highest weight vector $\frac{1}{2} z_{+}^{2}$ of $M\left(0,0 ; 2 ; y_{A}\right)$ the operator (2.14) gives the singular vector $w$. Hence, due to Remark 2.4 b, it suffices to check that $\nabla_{4}^{\prime}$ commutes with $f_{1}, f_{2}, f_{3}$. Commuting with $f_{3}$ gives the relations: $\left[f_{3}, a\right]=b,\left[f_{3}, b\right]=2 c$, which gives:

$$
b=d_{1}^{-} d_{2}^{+} d_{3}^{+}+d_{1}^{+} d_{2}^{-} d_{3}^{+}+d_{1}^{+} d_{2}^{+} d_{3}^{-}, c=d_{1}^{-} d_{2}^{-} d_{3}^{+}+d_{1}^{-} d_{2}^{+} d_{3}^{-}+d_{1}^{+} d_{2}^{-} d_{3}^{-} .
$$

With these $b$ and $c$, the operator $\nabla_{4}^{\prime}$ given by (2.14) clearly commutes with $s \ell(3)$. Thus, $\nabla_{4}^{\prime}$ : $M(0,0 ; 2 ;-2) \rightarrow M\left(0,1 ; 0 ;-\frac{2}{3}\right)$ defined by (2.14) and (2.15) is a non-zero homomorphism of $E(3,6)$-modules.

Another singular vector is the vector

$$
w_{2}=d_{1}^{-}\left(a \partial_{+}^{2}+b \partial_{+} \partial_{-}+c \partial_{-}^{2}\right)
$$

where $a, b, c$ are the same as in (2.14), (2.15), and $w_{2} \in M\left(0,0 ; 2 ; y_{D}\right)$. Similarly the checking is straightforward although somewhat tedious. Again this singular vector generates a nonzero homomorphism of $E(3,6)$-modules $\nabla_{4}^{\prime \prime}: M\left(1,0 ; 0 ; y_{A}\right) \rightarrow M\left(0,0 ; 2 ; y_{D}\right)$ such that for the highest weight vector $x_{1}$ of $M\left(1,0 ; 0 ; y_{A}\right)$ we have $\nabla_{4}^{\prime \prime} x_{1}=w_{2}$. This allows us to find that

$$
\begin{aligned}
\nabla_{4}^{\prime \prime}= & d_{1}^{-}\left(a \partial_{+}^{2}+b \partial_{+} \partial_{-}+c \partial_{-}^{2}\right) \partial_{1} \\
& +d_{2}^{-}\left(a \partial_{+}^{2}+b \partial_{+} \partial_{-}+c \partial_{-}^{2}\right) \partial_{2} \\
& +d_{3}^{-}\left(a \partial_{+}^{2}+b \partial_{+} \partial_{-}+c \partial_{-}^{2}\right) \partial_{3} .
\end{aligned}
$$


Clearly $\nabla_{4}^{\prime \prime}$ commutes with $f_{1}, f_{2}, f_{3}$ and Remark 2.4b shows that $\nabla_{4}^{\prime \prime}$ determines the morphism of $E(3,6)$-modules $\nabla_{4}^{\prime \prime}: M\left(1,0 ; 0 ; \frac{2}{3}\right) \rightarrow M(0,0 ; 2 ; 2)$.

The most complicated singular vector is $w_{3} \in M\left(0,0 ; 1 ; y_{D}\right)$, given by

$$
\begin{aligned}
w_{3}= & \left(\mathrm{d}_{123}^{-} \mathrm{d}_{123}^{+}+\left(\mathrm{d}_{13}^{-} \mathrm{d}_{12}^{+}-\mathrm{d}_{12}^{-} \mathrm{d}_{13}^{+}\right) \hat{\partial}_{1}+\left(\mathrm{d}_{21}^{-} \mathrm{d}_{23}^{+}-\mathrm{d}_{23}^{-} \mathrm{d}_{21}^{+}\right) \hat{\partial}_{2}+\right. \\
& +\left(\mathrm{d}_{32}^{-} \mathrm{d}_{31}^{+}-\mathrm{d}_{31}^{-} \mathrm{d}_{32}^{+}\right) \hat{\partial}_{3}-\mathrm{d}_{1}^{-} \mathrm{d}_{1}^{+} \hat{\partial}_{1}^{2}-\mathrm{d}_{2}^{-} \mathrm{d}_{2}^{+} \hat{\partial}_{2}^{2}-\mathrm{d}_{3}^{-} \mathrm{d}_{3}^{+} \hat{\partial}_{3}^{2}- \\
& \left.-\left(\mathrm{d}_{1}^{-} \mathrm{d}_{2}^{+}+\mathrm{d}_{2}^{-} \mathrm{d}_{1}^{+}\right) \hat{\partial}_{1} \hat{\partial}_{2}-\left(\mathrm{d}_{1}^{-} \mathrm{d}_{3}^{+}+\mathrm{d}_{3}^{-} \mathrm{d}_{1}^{+}\right) \hat{\partial}_{1} \hat{\partial}_{3}-\left(\mathrm{d}_{2}^{-} \mathrm{d}_{3}^{+}+\mathrm{d}_{3}^{-} \mathrm{d}_{2}^{+}\right) \hat{\partial}_{2} \hat{\partial}_{3}\right) \partial_{-}+ \\
& \quad+\left(\mathrm{d}_{1}^{-} \mathrm{d}_{123}^{+} \hat{\partial}_{1}+\mathrm{d}_{2}^{-} \mathrm{d}_{123}^{+} \hat{\partial}_{2}+\mathrm{d}_{3}^{-} \mathrm{d}_{123}^{+} \hat{\partial}_{3}\right) \partial_{+}= \\
= & \mathrm{d}_{123}^{-} a \partial_{-}+\left(\mathrm{d}_{1}^{-} \hat{\partial}_{1}+\mathrm{d}_{2}^{-} \hat{\partial}_{2}+\mathrm{d}_{3}^{-} \hat{\partial}_{3}\right)\left(b \partial_{-}+a \partial_{+}\right) .
\end{aligned}
$$

This singular vector generates a homomorphism of $E(3,6)$-modules

$$
\nabla_{6}: M\left(0,0 ; 1 ; y_{A}\right) \rightarrow M\left(0,0 ; 1 ; y_{D}\right)
$$

such that $\nabla_{6} z_{+}=w_{3}$. In (2.18) we keep the notations from (2.14), (2.15) and denote by $\hat{\partial}_{i}$ elements $\partial_{i} \in \mathrm{g}_{-2}(i=1,2,3)$.

It is straightforward to check that $\nabla w_{3}=0$, hence

$$
\nabla \cdot \nabla_{6}=0 .
$$

In Proposition 5.27 of Section 5 we will write $\nabla_{6}$ explicitely and show that $\nabla_{6} \cdot \nabla=0$ as well.

Remark 2.9. The operators $\nabla, \nabla_{2}, \nabla_{3}, \nabla_{4}^{\prime}, \nabla_{4}^{\prime \prime}$ and $\nabla_{6}$ have degree $1,2,3,4,4$ and 6 respectively, with respect to the $\mathbb{Z}$-gradation of $U\left(L_{-}\right)$induced by the consistent gradation of $E(3,6)$.

The maps $\nabla, \nabla_{2}, \nabla_{3}, \nabla_{4}^{\prime}, \nabla_{4}^{\prime \prime}$ and $\nabla_{6}$ are illustrated by Figure 1 . The black nods in quadrants $A, B, C, D$ represent generalized Verma modules $M\left(p, 0 ; r ; y_{X}\right), X=A, B$ or $M\left(0, q ; r ; y_{X}\right), X=C, D$. The plain arrows represent $\nabla$, the dotted arrows represent $\nabla_{2}$, the interrupted arrows represent $\nabla_{3}$ and the bold arrows represent $\nabla_{4}^{\prime}, \nabla_{4}^{\prime \prime}$ and $\nabla_{6}$.

There is still one more singular vector - the vector

$$
w_{4}=d_{1}^{+}\left(\Delta^{+} \partial_{+}+\Delta^{-} \partial_{-}\right)
$$

of the $E(3,6)$-module $M\left(0,1 ; 1 ; y_{D}\right)$. It occurs as the image of a non-trivial singular vector of $M(0,0 ; 0 ; 0)$ under the map $\nabla: M(0,0 ; 0 ; 0) \rightarrow M\left(0,1 ; 1 ; y_{D}\right)$ (cf. Corollary 2.5a for $p=r=0$ and $2.5 \mathrm{~d}$ for $q=r=1$ ). The reason for its existence is the fact that the composition of the following maps is non-zero:

$$
M\left(1,0 ; 1 ; y_{A}\right) \stackrel{\nabla}{\longrightarrow} M(0,0 ; 0 ; 0) \stackrel{\nabla}{\longrightarrow} M\left(0,1 ; 1 ; y_{D}\right) .
$$

The main result of [KR2] is the following theorem.

Theorem 2.10. A complete list of non-trivial singular vectors (up to a constant factor) of the $E(3,6)$-modules $M(p, q ; r ; y)$ is given by Corollary 2.5, Corollary 2.8, (2.17), (2.10), (2.18) and 2.29.

Corollary 2.11. All degenerate modules $M(p, q ; r ; y)$, except for $M\left(0,1 ; 1 ; \frac{2}{3}\right)$ (of type $\left.D\right)$, have a unique (up to a constant factor) non-trivial singular vector. The module $M\left(0,1 ; 1 ; \frac{2}{3}\right)$ has two such vectors. 


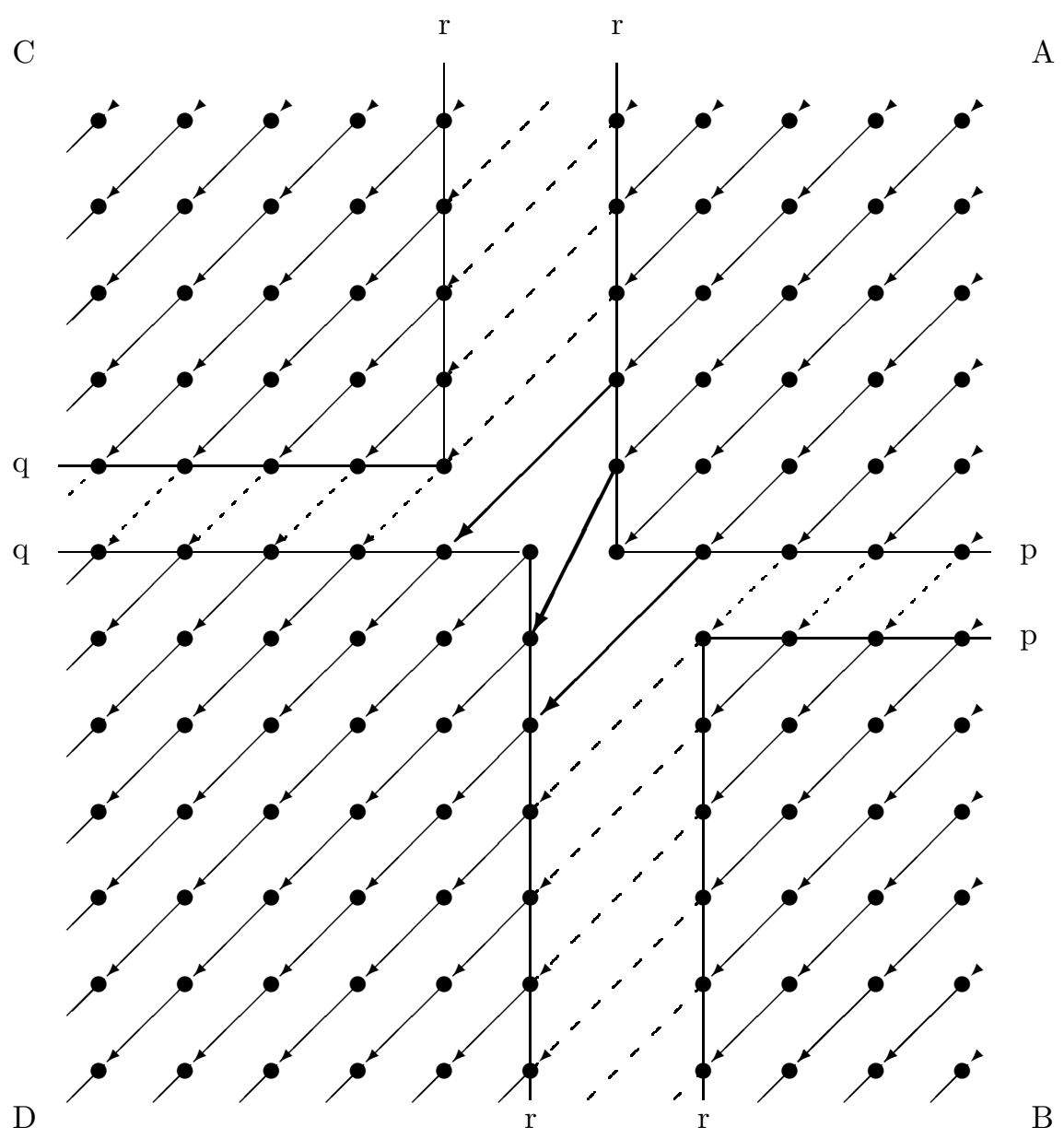

FiguRe 1.

3. Homology of complexes $\left(M_{X}, \nabla\right)$.

Recall that the complexes

$$
M_{X}=U\left(L_{-}\right) \otimes V_{X}
$$

are $\mathbb{Z}$-bigraded:

$$
M_{X}=\oplus_{m, n \in \mathbb{Z}} M_{X}^{m, n}, \quad M_{X}^{m, n}=U\left(L_{-}\right) \otimes V_{X}^{m, n},
$$

where $V_{X}^{m, n}=0$ if $X=A, m, n<0$; if $X=B, m<0, n>0$; if $X=C, m>0, n<0$; and if $X=D, m>0, n>0$, and $V_{X}^{m, n}$ are irreducible $\mathrm{g}_{0}$-modules described by (2.2) otherwise.

Note that

$$
\nabla: M_{X}^{m, n} \rightarrow M_{X}^{m-1, n-1} .
$$

Due to (3.2) the $\mathbb{Z}$-bigrading (3.1) of $M_{X}$ induces a $\mathbb{Z}$-bigrading on its homology:

$$
H\left(M_{X}\right)=\oplus_{m, n} H^{m, n}\left(M_{X}\right) .
$$


Note also that $M_{X}$ is a free $\mathrm{S}\left(\mathrm{g}_{-2}\right)$-module: $M_{X} \simeq \mathrm{S}\left(\mathrm{g}_{-2}\right) \otimes\left(\Lambda\left(\mathrm{g}_{-1}\right) \otimes V_{X}\right)$ and that $\nabla$ commutes with $\mathrm{S}\left(\mathrm{g}_{-2}\right)$. Hence $H\left(M_{X}\right)$ and each $H^{m, n}\left(M_{X}\right)$ are $\mathrm{S}\left(\mathrm{g}_{-2}\right)$-modules as well.

The canonical filtration of $U\left(L_{-}\right)$:

$$
\mathbb{C}=F_{0} U\left(L_{-}\right) \subset \ldots \subset F_{i} U\left(L_{-}\right)=L_{-} F_{i-1} U\left(L_{-}\right)+F_{i-1} U\left(L_{-}\right) \subset \ldots
$$

induces a filtration of $M_{X}$ by letting

$$
F_{i} M_{X}=F_{i} U\left(L_{-}\right) \otimes V_{X}
$$

Moreover $\nabla\left(F_{i} M_{X}\right) \subset F_{i+1} M_{X}$, so that $M_{X}$ becomes a filtered complex with the differential $\nabla$ and the bigrading (3.1) and the filtration is bounded below.

As we discuss in Appendix, one can use a spectral sequence to study the homology for such a complex and the spectral sequence converges when the filtration is bounded below. This applied to $\left(M_{X}, \nabla\right)$ produces a sequence of complexes $\left\{\left(E^{i}, \nabla^{i}\right)\right\}$, such that $E^{i+1}$ is the homology of $\left(E^{i}, \nabla^{i}\right), \lim _{i \rightarrow \infty} E^{i}=\operatorname{Gr}\left(H\left(M_{X}\right)\right)$, and $E^{0}=H\left(\operatorname{Gr} M_{X}\right)$.

Remark 3.1. In the subalgebra $W \simeq W_{3}$ described in (1.4) consider the standard filtration of $W_{3}: W=L_{-1}^{W} \supset L_{0}^{W} \supset \ldots$, then

$$
L_{j}^{W} \cdot F_{i} M_{X} \subset F_{i-j} M_{X} .
$$

Therefore the action of $W$ on $M_{X}$ descends to the action of $W$ on $\operatorname{Gr} M_{X}$, because $W \simeq$ Gr $W$. The actions commute with $\nabla$, and thus $W$ acts also on the spectral sequence and homologies.

To get hold on $H\left(\operatorname{Gr} M_{X}\right)$ we notice that, by the PBW theorem, the associated graded algebra $\operatorname{Gr} U\left(L_{-}\right) \simeq \mathrm{S}\left(\mathrm{g}_{-2}\right) \otimes \Lambda\left(\mathrm{g}_{-1}\right)$ (tensor product of associative algebras). Then we get the associated graded complex

$$
\operatorname{Gr} M_{X}=\operatorname{Gr} U\left(L_{-}\right) \otimes V_{X} \simeq\left(\mathrm{S}\left(\mathrm{g}_{-2}\right) \otimes \Lambda\left(\mathrm{g}_{-1}\right)\right) \otimes V_{X} .
$$

Clearly $L_{1}^{W}$ annihilates $\Lambda\left(\mathrm{g}_{-1}\right) \otimes V_{X}$ and the above isomorpism can be interpreted as the following isomorphism of $W$-modules

$$
\operatorname{Gr} M_{X} \simeq \mathrm{S}\left(\mathrm{g}_{-2}\right) \otimes\left(\Lambda\left(\mathrm{g}_{-1}\right) \otimes V_{X}\right) \simeq \operatorname{Ind}_{L_{0}^{W}}^{W}\left(\Lambda\left(\mathrm{g}_{-1}\right) \otimes V_{X}\right)
$$

The differential of the complex $\operatorname{Gr} M_{X}$ we denote again by $\nabla$, because it is given by the same formula as for $M_{X}$, except that the multiplication by $d_{i}^{ \pm}$is to be taken in $\operatorname{Gr} U\left(L_{-}\right)$ instead of the multiplication in $U\left(L_{-}\right)$.

It follows that $G_{X}=\Lambda\left(\mathrm{g}_{-1}\right) \otimes V_{X}$ is the subcomplex of the complex $\left(\mathrm{Gr} M_{X}, \nabla\right)$, and that the latter is obtained from the former by extending coefficients from $\mathbb{C}$ to $\mathrm{S}=\mathrm{S}\left(\mathrm{g}_{-2}\right)$. Homologies $H^{m, n}\left(G_{X}\right)$, that are computed with the differential $\nabla$ restricted from $\operatorname{Gr} M_{X}$, are also annihilated by $L_{1}^{W}$, so we have an isomorphism of $W$-modules (and $\mathrm{g}_{0}$-modules):

$$
H^{m, n}\left(\operatorname{Gr} M_{X}\right) \simeq \mathrm{S} \otimes_{\mathbb{C}} H^{m, n}\left(G_{X}\right) \simeq \operatorname{Ind}_{L_{0}^{W}}^{W}\left(H^{m, n}\left(G_{X}\right)\right),
$$

Thus, (3.6) and the theory of spectral sequence give us the following result:

Proposition 3.2. $H^{m, n}\left(G_{X}\right)=0 \Longrightarrow H^{m, n}\left(\operatorname{Gr} M_{X}\right)=0 \Longrightarrow H^{m, n}\left(M_{X}\right)=0$, and $\operatorname{rank}_{\mathrm{S}} H^{m, n}\left(M_{X}\right) \leq \operatorname{rank}_{\mathrm{S}} H^{m, n}\left(\operatorname{Gr} M_{X}\right)=\operatorname{dim}_{\mathbb{C}} H^{m, n}\left(G_{X}\right)$.

Figure 1 and Propositions 2.6, 2.7 make it reasonable to consider complexes not restricted to the quadrants of the figure. In the following we make the first step in this direction (see Figure 2 several pages below).

Let $M_{A B}$ be the module $M_{A} \oplus M_{B}$ with the bigrading and the filtration induced from the summands provided with the differential $\nabla$ that coincides with $\nabla_{2}$ on $M_{A^{\prime}} \subset M_{A} \oplus M_{B}$ and with $\nabla$ on the bigraded components of $M_{A} \oplus M_{B}$ that do not belong to $M_{A^{\prime}}$. 
Let us define $M_{C D}$ and also $G_{A B}, G_{C D}$ in the same way as the sum of the spaces with the differential constructed from $\nabla_{2}$ and $\nabla$.

Clearly $M_{A B}$ and $M_{C D}$ are filtered modules with the differential and the bigrading introduced above, and we can use the spectral sequence to study their homology. Moreover the isomorphism (3.6) holds for $X=A B, C D$ and Proposition 3.2 remains valid for $X=A B, C D$. This and the calculations of the homology $H^{m, n}\left(G_{X}\right)$ made in the next section provide us with the following proposition.

Proposition 3.3. $H^{m, n}\left(M_{A B}\right)=H^{m, n}\left(G_{A B}\right)=0$ when $m \geq 2$ or $m=1, n \neq 0,1,2$, $H^{m, n}\left(M_{C D}\right)=H^{m, n}\left(G_{C D}\right)=0$ when $m \leq-2$ or $m=-1, n \neq 0,-1,-2$.

\section{Homology of $G_{X}$.}

Let us notice that along with natural inclusions $V_{X^{\prime}} \subset V_{X}$ there are natural projections $V_{X} \rightarrow V_{X^{\prime}}$ defined by substituting $z_{ \pm}$and $\partial_{ \pm}$by zero. One has the corresponding projections $G_{X} \rightarrow G_{X^{\prime}}$.

We will consider the compositions $G_{X} \rightarrow G_{X^{\prime}} \rightarrow G_{Y^{\prime}}$ where the projection is the first map and $\nabla_{2}$ is the second one, as well as compositions $G_{X^{\prime}} \rightarrow G_{Y^{\prime}} \rightarrow G_{Y}$ where the first map is $\nabla_{2}$ and the second is the natural inclusion. These compositions are morphisms of $E(3,6)$-modules, and we allow ourselves to denote them by $\nabla_{2}$ as well.

Let

$$
\begin{array}{ll}
G_{A^{o}}=\operatorname{Ker}\left(\nabla_{2}: G_{A} \rightarrow G_{B^{\prime}}\right), & G_{B^{o}}=\operatorname{Coker}\left(\nabla_{2}: G_{A^{\prime}} \rightarrow G_{B}\right), \\
G_{C^{o}}=\operatorname{Ker}\left(\nabla_{2}: G_{C} \rightarrow G_{D^{\prime}}\right), & G_{D^{o}}=\operatorname{Coker}\left(\nabla_{2}: G_{C^{\prime}} \rightarrow G_{D}\right) .
\end{array}
$$

It follows from Proposition 2.6 a that the differential $\nabla$ is defined for $G_{X^{\circ}}$. It is clear from the above definitions that

$$
\begin{aligned}
& H^{m, n}\left(G_{A B}\right)= \begin{cases}H^{m, n}\left(G_{A^{o}}\right) & \text { for } n>0, \\
H^{m, n}\left(G_{B^{o}}\right) & \text { for } n<0, \\
H^{m, 0}\left(G_{A^{o}}\right) \oplus H^{m, 0}\left(G_{B^{\circ}}\right) & \text { for } n=0 ;\end{cases} \\
& H^{m, n}\left(G_{C D}\right)= \begin{cases}H^{m, n}\left(G_{C^{o}}\right) & \text { for } n>0, \\
H^{m, n}\left(G_{D^{o}}\right) & \text { for } n<0, \\
H^{m, 0}\left(G_{C^{o}}\right) \oplus H^{m, 0}\left(G_{D^{o}}\right) & \text { for } n=0 .\end{cases}
\end{aligned}
$$

This means that the computation of the homologies $H^{m, n}\left(G_{A B}\right), H^{m, n}\left(G_{C D}\right)$ reduces to finding $H^{m, n}\left(G_{X^{o}}\right)$.

In the computations we need to go from $G_{X}$ to $G_{X^{\circ}}$ and the following lemma is helpful.

Lemma 4.1. Let $(M, d)$ be a differential complex

$$
M_{0} \leftarrow M_{1} \leftarrow M_{2} \leftarrow \cdots,
$$

let $(N, d)$ be another differential complex of the same type and let $\alpha: M \rightarrow N$ be a morphism of complexes.

(1) Suppose $M^{o}=\operatorname{Ker} \alpha$ and $N$ is concentrated at 0 (i.e., $N_{i}=0$ for $i \neq 0$ ). Then $H_{n}(M) \simeq H_{n}\left(M^{o}\right)$ for $n \neq 0$ and there is an exact sequence:

$$
0 \rightarrow H_{0}\left(M^{o}\right) \rightarrow H_{0}(M) \rightarrow H_{0}(N) .
$$

(2) Suppose $N^{o}=$ Coker $\alpha$ and $M$ is concentrated at 0 . Then $H_{n}(N) \simeq H_{n}\left(N^{o}\right)$ for $n \neq 0$ and there is an exact sequence:

$$
H_{n}(M) \rightarrow H_{n}(N) \rightarrow H_{n}\left(N^{o}\right) \rightarrow 0 .
$$


The statement follows immediately from definitions.

We can apply this lemma to connect the $\nabla$-homology of $G_{X^{\circ}}$ and $G_{X}$ since one can check, using the definitions (4.1), (4.2), that the conditions of the lemma are valid.

In the rest of the section we also consider the following $\mathbb{Z}$-bigrading of modules $G_{X}$. Let for $X=A, B, C, D$

$$
\begin{aligned}
& \left(V_{X}\right)_{[p, q]}=\left\{f \in V_{X} \mid\left(z_{+} \partial_{+}\right) f=p f,\left(z_{-} \partial_{-}\right) f=q f\right\}, \text { and } \\
& \left(G_{X}\right)_{[p, q]}=\Lambda\left(g_{-1}\right) \otimes\left(V_{X}\right)_{[p, q]},
\end{aligned}
$$

It is important to mention that $\left(G_{X}^{m, n}\right)_{[p, q]} \neq 0$ only if $p+q=n$.

We hope that the notations allow one to distinguish what grading is refered to. The new bigrading naturally descends to $G_{X^{\circ}}$.

The definitions of $\Delta^{ \pm}$, Lemma 2.1c and the formula

$$
\nabla=\Delta^{+} \partial_{+}+\Delta^{-} \partial_{-}
$$

allow us to conclude that the bigraded modules $G_{X}, G_{X^{\circ}}$ provided with differentials $\mathrm{d}^{\prime}=$ $\Delta^{+} \partial_{+}, \mathrm{d}^{\prime \prime}=\Delta^{-} \partial_{-}$become bicomplexes and their homologies with respect to $\nabla$ (that we are interested in) are nothing but the total homoligies of the bicomplexes. So the classical theory of spectral sequences of a bicomplex is relevant here ([ML, Chapter XI, section 6]) and the following lemma contains well-known statements about two spectral sequences of a bicomplex that we will use.

Lemma 4.2. Let $\left(K, \mathrm{~d}^{\prime}, \mathrm{d}^{\prime \prime}\right)$ be a bicomplex, $K=\sum_{p, q} K_{[p, q]}$, and $\mathrm{d}=\mathrm{d}^{\prime}+\mathrm{d}^{\prime \prime}$ the total differential of $K$.

The first spectral sequence of the bicomplex $E^{\prime}=\left\{\left(E^{\prime r}, d^{r}\right)\right\}, E^{\prime r}=\sum_{p, q} E_{[p, q]}^{\prime r}$ has the property:

$$
\left(E^{\prime 0}, d^{0}\right) \simeq\left(K, \mathrm{~d}^{\prime \prime}\right), \quad\left(E^{\prime 1}, d^{1}\right) \simeq\left(H\left(K, \mathrm{~d}^{\prime \prime}\right), \mathrm{d}^{\prime}\right), \text { so that } E_{[p, q]}^{2} \simeq H_{p}\left(H_{q}\left(K, \mathrm{~d}^{\prime \prime}\right), \mathrm{d}^{\prime}\right) .
$$

For the second spectral sequence $E^{\prime \prime}=\left\{\left(E^{\prime \prime r}, d^{r}\right)\right\}, E^{\prime \prime r}=\sum_{p, q} E_{[p, q]}^{\prime r}$ the roles of $\mathrm{d}^{\prime}, \mathrm{d}^{\prime \prime}$ are reversed:

$$
\left(E^{\prime \prime 0}, d^{0}\right) \simeq\left(K, \mathrm{~d}^{\prime}\right), \quad\left(E^{\prime \prime 1}, d^{1}\right) \simeq\left(H\left(K, \mathrm{~d}^{\prime}\right), \mathrm{d}^{\prime \prime}\right), \text { so that } E_{[p, q]}^{\prime 2} \simeq H_{p}\left(H_{q}\left(K, \mathrm{~d}^{\prime}\right), \mathrm{d}^{\prime \prime}\right) .
$$

The spectral sequences are functors on the bicomplexes.

Any of the above spectral sequences $E$ converges to the homology of $K$ with respect to the total differential $\mathrm{d}$ whenever for every $n$ the set

$$
\left\{(p, q) \mid p+q=n, E_{[p, q]}^{2} \neq 0\right\}
$$

is finite.

The above condition for the convegence could be relaxed but this form is enough for our purposes. It is farely traditional although slightly differs from the one in Theorem 6.1 of [ML, Chapter XI]. The arguments of Proposition 3.2 of [ML, Chapter XI] are easily modified to prove the convegence under our condition. We leave details to the reader.

Now we decompose bicomplexes $G_{X}$ ( and their sub- or quotient-bicomplexes $G_{X^{\circ}}$ ) into a sum of smaller bicomplexes.

Introduce notations:

$$
\Lambda_{i}^{ \pm}=\Lambda^{i}\left\langle d_{1}^{ \pm}, d_{3}^{ \pm}, d_{3}^{ \pm}\right\rangle
$$

and $(0 \leq i, j \leq 3)$ :

$$
\Lambda_{i}^{+} \Lambda_{j}^{-}[x]=\Lambda_{i}^{+} \Lambda_{j}^{-} \otimes_{\mathbb{C}} \mathbb{C}\left[x_{1}, x_{2}, x_{3}\right], \quad \Lambda_{i}^{+} \Lambda_{j}^{-}[\partial]=\Lambda_{i}^{+} \Lambda_{j}^{-} \otimes_{\mathbb{C}} \mathbb{C}\left[\partial_{1}, \partial_{2}, \partial_{3}\right] .
$$


Let

$$
\begin{array}{ll}
G_{A}(a, b)_{[p, q]}=\Lambda_{a-p}^{+} \Lambda_{b-q}^{-}[x] z_{+}^{p} z_{-}^{q}, & G_{C}(a, b)_{[p, q]}=\Lambda_{a-p}^{+} \Lambda_{b-q}^{-}[\partial] z_{+}^{p} z_{-}^{q}, \\
G_{B}(a, b)_{[p, q]}=\Lambda_{a-p}^{+} \Lambda_{b-q}^{-}[x] \partial_{+}^{-p} \partial_{-}^{-q}, & G_{D}(a, b)_{[p, q]}=\Lambda_{a-p}^{+} \Lambda_{b-q}^{-}[\partial] \partial_{+}^{-p} \partial_{-}^{-q} .
\end{array}
$$

Then $G_{X}$ decomposes in a direct sum of subcomplexes:

$$
G_{X}=\oplus_{a, b} G_{X}(a, b), \text { where } G_{X}(a, b)=\bigoplus_{p, q} G_{X}(a, b)_{[p, q]} .
$$

We have the induced decomposition $G_{X^{o}}=\oplus_{a, b} G_{X^{\circ}}(a, b)$.

Note that the above equalities are isomorphisms of $s \ell(3)$-modules but not those of $s \ell(2)$ modules, since $G_{X}(a, b)=\left\{f \in V_{X} \mid\left(z_{+} \partial_{+}\right) f=a f,\left(z_{-} \partial_{-}\right) f=b f\right\}$ with the obvious action of $z_{ \pm} \partial_{ \pm}$on $G_{X}$.

Thus, in order to know $H^{m, n}\left(G_{X^{o}}\right)$ it is enough to compute homology $H^{m, n}\left(G_{X^{o}}(a, b)\right)$ and this is our further goal. We will use formulae (4.6), (4.7) in these computations.

Let us consider $s \ell(3)$-modules $\Lambda^{i}=\Lambda^{i}\left\langle x_{1}, x_{2}, x_{3}\right\rangle$ for $i \geq 0$, and let $\Lambda^{i}=0$ for $i<0$. Of course $\Lambda^{i}=0$ for $i>3$ too.

Proposition 4.3. If $a, b>3$, then (as $s \ell(3)$-modules)

$$
\begin{aligned}
& H^{m, n}\left(G_{A^{o}}(a, b), \nabla\right)=H^{m, n}\left(G_{A}(a, b), \nabla\right) \simeq\left\{\begin{array}{lll}
0, & \text { for } & m>0, \\
\Lambda^{a+b-n}, & \text { for } & m=0 ;
\end{array}\right. \\
& H^{m, n}\left(G_{C^{o}}(a, b), \nabla\right)=H^{m, n}\left(G_{C}(a, b), \nabla\right) \simeq\left\{\begin{array}{lll}
0, & \text { for } & m<0, \\
\Lambda^{a+b-n-3}, & \text { for } & m=0 .
\end{array}\right.
\end{aligned}
$$

If $a, b<0$, then (as $s \ell(3)$-modules)

$$
\begin{gathered}
H^{m, n}\left(G_{B^{\circ}}(a, b), \nabla\right)=H^{m, n}\left(G_{B}(a, b), \nabla\right) \simeq\left\{\begin{array}{lll}
0, & \text { for } & m>0, \\
\Lambda^{a+b-n}, & \text { for } & m=0,
\end{array}\right. \\
H^{m, n}\left(G_{D^{o}}(a, b), \nabla\right)=H^{m, n}\left(G_{D}(a, b), \nabla\right) \simeq\left\{\begin{array}{lll}
0, & \text { for } & m<0, \\
\Lambda^{a+b-n-3}, & \text { for } & m=0 .
\end{array}\right.
\end{gathered}
$$

(Note that for $X=B$ or $D$ the above formulae show that the homology $H^{0, n}\left(G_{X^{o}}(a, b), \nabla\right)$ can be non-zero only for negative $n$, namely when

$-3+a+b \leq n \leq a+b<0$ for $B$ and $-6+a+b \leq n \leq-3+a+b<0$ for $D$.)

Proof. First of all it follows from (4.6) that, under restrictions of the proposition, $G_{X}=$ $G_{X^{o}}$, because we are to care about the difference only if $\left(G_{X}\right)_{[0,0]} \neq 0$ which is not the case here.

We use the spectral sequences of Lemma 4.2 for the evaluation of $H\left(G_{X}\right)$ and the first spectral sequence happens to be sufficient for the proof. As we compute the $E^{\prime 2}$-term, we notice that we have got a one-row spectral sequence that necessarily degenerates (i.e. all the higher differentials are zero), thus ${E^{\prime}}^{2} \simeq{E^{\prime}}^{\infty} \simeq H\left(G_{X}, \nabla\right)$ as $s \ell(3)$-modules.

Let us start with the $A$-case. By Lemma 4.2

$$
{E^{\prime}}_{[p, q]}^{2}\left(G_{A}(a, b)\right) \simeq H_{p}\left(H_{q}\left(G_{A}(a, b), \mathrm{d}^{\prime \prime}\right), \mathrm{d}^{\prime}\right),
$$

thus we are to begin with considering $G_{A}(a, b)$ as a complex with the differential d" $=\Delta^{-} \partial_{-}$.

We see from (4.6) that it splits into a sum of subcomplexes

$$
\cdots \leftarrow \Lambda_{a-p}^{+} \Lambda_{b-q+1}^{-}[x] z_{+}^{p} z_{-}^{q-1} \longleftarrow \Lambda_{a-p}^{+} \Lambda_{b-q}^{-}[x] z_{+}^{p} z_{-}^{q} \leftarrow \cdots
$$


Observing how the differential acts we conclude that the complex (4.8) is isomorphic to the tensor product of the following complex

$$
0 \leftarrow \Lambda_{3}^{-}[x] z_{-}^{b-3} \leftarrow \cdots \leftarrow \Lambda_{b-q+1}^{-}[x] z_{-}^{q-1} \longleftarrow \Lambda_{b-q}^{-}[x] z_{-}^{q} \leftarrow \cdots \leftarrow \Lambda_{0}^{-}[x] z_{-}^{b} \leftarrow 0
$$

(note that $b>3$ ) with the vector space $\Lambda_{a-p}^{+} z_{+}^{p}$ which is not affected by the differential.

The complex (4.9) is nothing but a De Rham complex with the "grading variable" $z_{-}$ added. This implies that it has non-zero homologies only at its right end (with our direction of arrows), and those are isomorphic to $\mathbb{C} z_{-}^{b}$.

Therefore the complex (4.8) also has its non-zero homologies only at one place and those are isomorphic to $\Lambda_{a-p}^{+} z_{+}^{p} z_{-}^{b}$.

This shows us that all non-zero terms in ${E^{\prime}}_{[p, q]}^{1}\left(G_{A}(a, b)\right)$ are confined to one row $q=b$ and that $\mathrm{d}^{\prime}$ is zero on this row. Thus ${E^{\prime 2}}^{2}={E^{\prime}}^{1}$ and for a one-row spectral sequence $E^{\prime 2}=\ldots=E^{\prime \infty}$, hence we have arrived at the following answer

$$
{E^{\prime}}_{[p, q]}^{\infty}\left(G_{A}(a, b)\right)=\left\{\begin{array}{ccc}
0 & \text { for } & q \neq b, \\
\Lambda_{a-p}^{+} z_{+}^{p} z_{-}^{b} & \text { for } & q=b .
\end{array}\right.
$$

At the same time

$$
\sum_{m} H^{m, n}\left(G_{A}(a, b)\right) \simeq \sum_{p+q=n} E_{[p, q]}^{\infty}\left(G_{A}(a, b)\right) \simeq E_{[n-b, b]}^{\prime \infty}\left(G_{A}(a, b)\right) \simeq \Lambda_{a+b-n}^{+} z_{+}^{n-b} z_{-}^{b} .
$$

Now (4.11) shows that $H^{m, n}\left(G_{A}(a, b)\right)=0$ for $m \neq 0$ and that it has the stated value for $m=0$. This proves the proposition for $X=A$.

The proof for $X=B$ is quite similar, we are to deal with complexes

$$
\cdots \leftarrow \Lambda_{a-p}^{+} \Lambda_{b-q+1}^{-}[x] \partial_{+}^{-p} \partial_{-}^{1-q} \longleftarrow \Lambda_{a-p}^{+} \Lambda_{b-q}^{-}[x] \partial_{+}^{-p} \partial_{-}^{-q} \leftarrow \cdots
$$

isomorphic to the tensor product of a De Rham complex

$$
0 \leftarrow \Lambda_{3}^{-}[x] \partial_{-}^{3-b} \leftarrow \cdots \leftarrow \Lambda_{b-q+1}^{-}[x] \partial_{-}^{1-q} \longleftarrow \Lambda_{b-q}^{-}[x] \partial_{-}^{-q} \leftarrow \cdots \leftarrow \Lambda_{0}^{-}[x] \partial_{-}^{-b} \leftarrow 0
$$

$(b<0)$, and a vector space $\Lambda_{a-p}^{+} \partial_{+}^{-p}$ which is not affected by the differential.

It gives

$$
E_{[p, q]}^{2}\left(G_{B}(a, b)\right)=\left\{\begin{array}{ccc}
0 & \text { for } & q \neq b \\
\Lambda_{a-p}^{+} \partial_{+}^{-p} \partial_{-}^{-b} & \text { for } & q=b .
\end{array}\right.
$$

We have got the same configuration with one non-zero row, hence $E^{\prime 2}=\ldots=E^{\prime \infty}$. At the same time

$$
\sum_{m} H^{m, n}\left(G_{B}(a, b)\right) \simeq \sum_{p+q=n}{E^{\prime}}_{[p, q]}^{\infty}\left(G_{B}(a, b)\right) \simeq E_{[n-b, b]}^{\infty}\left(G_{B}(a, b)\right) \simeq \Lambda_{a+b-n}^{+} \partial_{+}^{b-n} \partial_{-}^{-b},
$$

and this immediately implies the proposition for $X=B$.

Going to $X=C$ we represent $G_{C}(a, b)$ as the sum of subcomplexes of the form

$$
\cdots \leftarrow \Lambda_{a-p}^{+} \Lambda_{b-q+1}^{-}[\partial] z_{+}^{p} z_{-}^{q-1} \longleftarrow \Lambda_{a-p}^{+} \Lambda_{b-q}^{-}[\partial] z_{+}^{p} z_{-}^{q} \leftarrow \cdots
$$

which differs from (4.8) in that respect that here we have modules over $\mathbb{C}[\partial]$ instead of $\mathbb{C}[x]$. Again the complex (4.16) is isomorphic to the tensor product of

$$
0 \leftarrow \Lambda_{3}^{-}[\partial] z_{-}^{b-3} \leftarrow \cdots \leftarrow \Lambda_{b-q+1}^{-}[\partial] z_{-}^{q-1} \longleftarrow \Lambda_{b-q}^{-}[\partial] z_{-}^{q} \leftarrow \cdots \leftarrow \Lambda_{0}^{-}[\partial] z_{-}^{b} \leftarrow 0
$$

$(b>3)$, and a vector space $\Lambda_{a-p}^{+} z_{+}^{p}$ which is not affected by the differential. 
Now with its differential the complex (4.17) is essentially a Koszul complex (dual of the De Rham complex) and this implies that it has non-zero homologies only at its very left end (with our direction of arrows), and those are isomorphic to $\Lambda_{3}^{-} z_{-}^{b-3}$. Thus the complex (4.16) also has its non-zero homologies only at its left end and those are isomorphic to $\Lambda_{a-p}^{+} \Lambda_{3}^{-} z_{+}^{p} z_{-}^{b-3}$.

We have got a one-row spectral sequence again, but with the row $q=b-3$ and again $\mathrm{d}^{\prime}$ is zero on this row. Hence

$$
{E^{\prime}}_{[p, q]}^{2}\left(G_{C}(a, b)\right)=\left\{\begin{array}{ccc}
0 & \text { for } & q \neq b-3 \\
\Lambda_{a-p}^{+} \Lambda_{3}^{-} z_{+}^{p} z_{-}^{b-3} & \text { for } & q=b-3 .
\end{array}\right.
$$

Now $E_{[p, q]}^{\prime 2}=E_{[p, q]}^{\prime \infty}$,

$$
\sum_{m} H^{m, n}\left(G_{C}(a, b)\right) \simeq{E^{\prime n-b+3, b-3]}}^{\infty}\left(G_{C}(a, b)\right) \simeq \Lambda_{a+b-n-3}^{+} \Lambda_{3}^{-} z_{+}^{n-b+3} z_{-}^{b-3},
$$

and, taking into account the isomorphism $\Lambda_{3}^{-} \simeq \mathbb{C}$, we conclude that we proved the proposition for $X=C$.

For $X=D$ we arrive similarly at the complex

$$
\cdots \leftarrow \Lambda_{a-p}^{+} \Lambda_{b-q+1}^{-}[\partial] \partial_{+}^{-p} \partial_{-}^{1-q} \longleftarrow \Lambda_{a-p}^{+} \Lambda_{b-q}^{-}[\partial] \partial_{+}^{-p} \partial_{-}^{-q} \leftarrow \cdots,
$$

which is the tensor product of a Koszul complex

$$
0 \leftarrow \Lambda_{3}^{-}[\partial] \partial_{-}^{3-b} \leftarrow \cdots \leftarrow \Lambda_{b-q+1}^{-}[\partial] \partial_{-}^{1-q} \longleftarrow \Lambda_{b-q}^{-}[\partial] \partial_{-}^{-q} \leftarrow \cdots \leftarrow \Lambda_{0}^{-}[\partial] \partial_{-}^{-b} \leftarrow 0
$$

$(b<0)$, and a vector space $\Lambda_{a-p}^{+} \partial_{+}^{-p}$. So we get a formula

$$
{E^{\prime}}_{[p, q]}^{2}\left(G_{D}(a, b)\right)=\left\{\begin{array}{ccc}
0 & \text { for } & q \neq b-3 \\
\Lambda_{(a-p)}^{+} \Lambda_{3}^{-} \partial_{+}^{-p} \partial_{-}^{3-b} & \text { for } & q=b-3 .
\end{array}\right.
$$

Again ${E^{\prime}}_{[p, q]}^{2}=E_{[p, q]}^{\prime \infty}$, and

$$
\sum_{m} H^{m, n}\left(G_{D}(a, b)\right) \simeq{E^{\prime}}_{[n-b+3, b-3]}^{\infty}\left(G_{D}(a, b)\right) \simeq \Lambda_{a+b-n-3}^{+} \Lambda_{3}^{-} \partial_{+}^{b-n-3} \partial_{-}^{3-b} .
$$

This is enough to prove the proposition for $X=D$.

Looking back at the proof we notice that the roles of $a$ and $b$ are not symmetric. The restrictions on $b$ are important in that respect that the complexes (4.9), (4.13), (4.16), (4.21) would not be cut short and remain the full length De Rham or Koszul complexes. But the restrictions on $a$ have not been used.

Therefore leaving the proof the same we can relax the rectrictions on $a$, arriving at the following corollary.

Corollary 4.4. If $b>3$, then

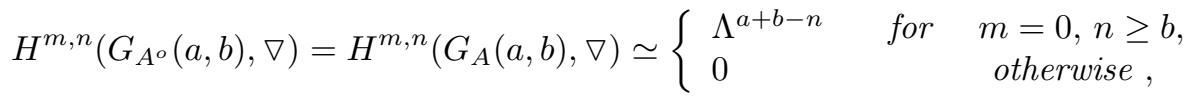

$$
\begin{aligned}
& H^{m, n}\left(G_{C^{o}}(a, b), \nabla\right)=H^{m, n}\left(G_{C}(a, b), \nabla\right) \simeq\left\{\begin{array}{lll}
\Lambda^{a+b-n-3} & \text { for } & m=0, n \geq b-3 . \\
0 & \text { otherwise } .
\end{array}\right.
\end{aligned}
$$

If $b<0$, then

$$
\begin{aligned}
& H^{m, n}\left(G_{B^{o}}(a, b), \nabla\right)=H^{m, n}\left(G_{B}(a, b), \nabla\right) \simeq\left\{\begin{array}{lr}
\Lambda^{a+b-n} & \text { for } \\
0 & \text { otherwise },
\end{array}\right. \\
& H^{m, n}\left(G_{D^{o}}(a, b), \nabla\right)=H^{m, n}\left(G_{D}(a, b), \nabla\right) \simeq\left\{\begin{array}{lrr}
\Lambda^{a+b-n-3} & \text { for } & m=0, n \leq b-3 \\
0 & \text { otherwise } .
\end{array}\right.
\end{aligned}
$$


Corollary 4.5. By interchanging a and $b$ in Corollary 4.4 we get valid statements as well.

This is because we can use the second spectral sequence of Lemma 4.2 in the proof instead of the first one.

We also have to remember that for some values of $a$ complexes $G_{X}(a, b)$ are entirely zero. Thus we can assume that $a \geq 0$ for $X=A, A^{o}, C, C^{o}$ and $a \leq 3$ for $X=C, C^{o}, D, D^{o}$, and we are left with the cases when $0 \leq a, b \leq 3$. Here the answer becomes somewhat different and the proof demands more elaborate arguments, but goes along the same lines of computing the homology via spectral sequence.

Proposition 4.6. Let $0 \leq a \leq b \leq 3$ then

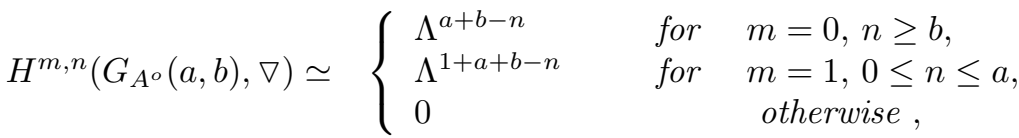

$$
\begin{aligned}
& H^{m, n}\left(G_{C^{o}}(a, b), \nabla\right) \simeq\left\{\begin{array}{lr}
\Lambda^{a+b-n-3} & \text { for } \quad m=0, n \geq 0 \\
0 & \text { otherwise }
\end{array}\right. \\
& H^{m, n}\left(G_{B^{\circ}}(a, b), \nabla\right) \simeq\left\{\begin{array}{lr}
\Lambda^{a+b-n} \\
0
\end{array} \quad \text { for } \begin{array}{c}
m=0, n \leq 0, \\
\text { otherwise } .
\end{array}\right.
\end{aligned}
$$

Let $0 \leq b \leq a \leq 3$ then

$$
H^{m, n}\left(G_{D^{o}}(a, b), \nabla\right) \simeq\left\{\begin{array}{lll}
\Lambda^{a+b-n-3} & \text { for } & m=0, n \leq b-3 \\
\Lambda^{-1+a+b-n-3} & \text { for } & m=-1, a-3 \leq n \leq 0 . \\
0 & & \text { otherwise. }
\end{array}\right.
$$

Proof. We see immediately that the statement is true for the following complexes

$$
\begin{array}{ll}
G_{A^{o}}(0,0)=\mathbb{C}+\left\langle x_{1}, x_{2}, x_{3}\right\rangle, & G_{C^{o}}(0,0)=0, \\
G_{B^{\circ}}(3,3)=0, & G_{D^{o}}(3,3)=\mathbb{C}+\left\langle\partial_{1}, \partial_{2}, \partial_{3}\right\rangle,
\end{array}
$$

with trivial differentials. We exclude these cases from consideration further on.

We already noticed that variables $z_{ \pm}$(resp. $\partial_{ \pm}$) play only the role of grading variables, so we can eliminate them as it is done below.

Let us define a bicomplex

$$
\left(\widetilde{G}_{A}(a, b)\right)_{[p, q]}=\left\{\begin{array}{cc}
\Lambda_{a-p}^{+} \Lambda_{b-q}^{-}[x] & \text { for } p \geq 0, q \geq 0 \\
0 & \text { overwise }
\end{array}\right.
$$

with the differentials $\mathrm{d}^{\prime}=\Delta^{+}, \mathrm{d}^{\prime \prime}=\Delta^{-}$. Comparing with (4.6) we conclude that there exists an isomorphism of bicomplexes $\alpha: G_{A}(a, b) \rightarrow \widetilde{G}_{A}(a, b)$.

Let

$$
\left(G_{B^{\prime}}(a, b)\right)_{[p, q]}=\left\{\begin{array}{cc}
\Lambda_{a+1}^{+} \Lambda_{b+1}^{-}[x] & \text { for } p=0, q=0, \\
0 & \text { overwise. }
\end{array}\right.
$$

Following (4.1) the bicomplex $G_{A^{\circ}}(a, b)$ is the kernel of the morphism $\nabla_{2}: G_{A}(a, b) \rightarrow$ $G_{A^{\prime}}(a, b)$ and the morphism can be included into a commutative diagram

$$
\begin{array}{ccc}
G_{A}(a, b) & \longrightarrow & G_{A^{\prime}}(a, b) \\
\downarrow & & \| \\
\widetilde{G}_{A}(a, b) & \longrightarrow & G_{A^{\prime}}(a, b)
\end{array}
$$


where the morphism at the lower row is equal to $\Delta^{+} \Delta^{-}$. This shows that $\alpha$ induces an isomorphism between $G_{A^{o}}(a, b)$ and the bicomplex

$$
\widetilde{G}_{A^{o}}(a, b)=\operatorname{Ker}\left(\Delta^{+} \Delta^{-}: \widetilde{G}_{A}(a, b) \longrightarrow G_{A^{\prime}}(a, b)\right),
$$

thus it also induces an isomorphism of their homologies.

The bicomplex $\widetilde{G}_{A}(a, b)$ can be represented by the following diagram (we allow ourselves to omit the zero components and morphisms with a zero source or target):

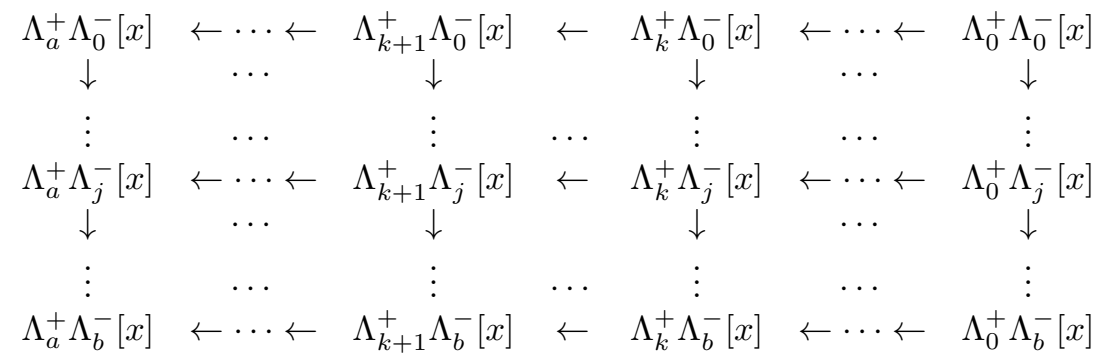

where the row (resp. column) maps are the De Rham differentials $\Delta^{+}$(resp. $\Delta^{-}$).

To represent $\widetilde{G}_{A^{\circ}}(a, b)$ by a similar diagram we need only to change it in the lower-left corner and put there $\operatorname{Ker}\left(\Delta^{+} \Delta^{-}\right)$.

It follows that the $E^{\prime 1}$-term of the spectral sequence of the bicomplex $\widetilde{G}_{A^{\circ}}(a, b)$ is represented by the following diagram:

$$
\begin{array}{ccccccc}
\Lambda_{a}^{+} & \leftarrow \cdots \leftarrow & \Lambda_{k+1}^{+} & \leftarrow & \Lambda_{k}^{+} & \leftarrow \cdots \leftarrow & \Lambda_{0}^{+} \\
0 & \leftarrow \cdots \leftarrow & 0 & \leftarrow & 0 & \leftarrow \cdots \leftarrow & 0 \\
\cdots & \cdots \cdots & \cdots & \cdots & \cdots & \cdots \cdots & \cdots \\
0 & \leftarrow \cdots \leftarrow & 0 & \leftarrow & 0 & \leftarrow \cdots \leftarrow & 0 \\
\frac{\operatorname{Ker} \Delta^{+} \Delta^{-}}{\operatorname{Im} \Delta^{-}} & \leftarrow \cdots \leftarrow & \frac{\Lambda_{k+1}^{+} \Lambda_{b}^{-}[x]}{\operatorname{Im} \Delta^{-}} & \leftarrow & \frac{\Lambda_{k}^{+} \Lambda_{b}^{-}[x]}{\operatorname{Im} \Delta^{-}} & \leftarrow \cdots \leftarrow & \frac{\Lambda_{0}^{+} \Lambda_{b}^{-}[x]}{\operatorname{Im} \Delta^{-}}
\end{array}
$$

with only two non-zero rows, those where $q=0$ and $q=b$. Here $b \neq 0$ as $0 \leq a \leq b$ and we have excluded $a=b=0$.

The $E^{\prime 2}$-term will have the similar "two rows" structure, and this together with $a \leq b$ imply that for every differential $d_{[p, q]}^{r}, r \geq 2$, either its source or its target is zero. Thus all the differentials are trivial and therefore $E^{\prime 2}=\cdots=E^{\prime \infty}$.

Let us compute $E^{\prime 2}$. First of all the differential $d^{\prime}$ is induced by $\Delta^{+}$and evidently it is trivial on the upper row, hence the row descends to $E^{\prime 2}$ unchanged.

The following lemma helps us to compute the terms in the lower row.

Lemma 4.7. Let $R(a, b)$ be the complex

$$
\frac{\Lambda_{0}^{+} \Lambda_{b}^{-}[x]}{\operatorname{Im} \Delta^{-}} \stackrel{\Delta^{+}}{\longrightarrow} \quad \frac{\Lambda_{1}^{+} \Lambda_{b}^{-}[x]}{\operatorname{Im} \Delta^{-}} \stackrel{\Delta^{+}}{\longrightarrow} \cdots \stackrel{\Delta^{+}}{\longrightarrow} \frac{\Lambda_{k}^{+} \Lambda_{b}^{-}[x]}{\operatorname{Im} \Delta^{-}} \stackrel{\Delta^{+}}{\longrightarrow} \cdots \stackrel{\Delta^{+}}{\longrightarrow} \frac{\operatorname{Ker} \Delta^{+} \Delta^{-}}{\operatorname{Im} \Delta^{-}}
$$

and $S(a, b)$ be the complex

$$
\Delta^{-}\left(\Lambda_{0}^{+} \Lambda_{b}^{-}[x]\right) \stackrel{\Delta^{+}}{\rightarrow} \Delta^{-}\left(\Lambda_{1}^{+} \Lambda_{b}^{-}[x]\right) \stackrel{\Delta^{+}}{\rightarrow} \cdots \stackrel{\Delta^{+}}{\rightarrow} \quad \Delta^{-}\left(\Lambda_{k}^{+} \Lambda_{b}^{-}[x]\right) \stackrel{\Delta^{+}}{\rightarrow} \cdots \stackrel{\Delta^{+}}{\rightarrow} S(a, b)_{b},
$$

where the last term $S(a, b)_{b}=\operatorname{Ker}\left(\Delta^{-}\left(\Lambda_{a}^{+} \Lambda_{b}^{-}[x]\right) \stackrel{\Delta^{+}}{\rightarrow} \Delta^{-}\left(\Lambda_{a+1}^{+} \Lambda_{b}^{-}[x]\right)\right)$.

Then

(a) $\Delta^{-}$induces an isomorphism of complexes $R(a, b) \rightarrow S(a, b)$ for $b>0$, 
(b) homologies of $R(a, b)$ (and $S(a, b)$ ) for $b>0$ are isomorphic to:

$$
\Lambda^{b+1}, \quad \Lambda^{b+2}, \quad \ldots \quad, \quad \Lambda^{b+k+1}, \quad \ldots \quad, \quad \Lambda^{a+b+1} .
$$

In the notations of Lemma 4.7 we write the terms of the lower non-trivial row of the $E^{\prime 2}$-term of the spectral sequence as follows:

$$
{E^{\prime}}_{[p, 0]}^{2}\left(\widetilde{G}_{A^{o}}(a, b)\right)=H_{a-p}(R(a, b))
$$

(in the part (b) of the lemma these terms are explicitely calculated).

Proof of Lemma 4.7. The statement (a) is obvious. Going to (b) let us first of all notice that the second complex has a nice property:

$$
H_{i}(S(a, b))=H_{i}(S(a+1, b)) \text { for } 0 \leq i \leq a .
$$

Thus it is sufficient to compute its homologies for large $a$, and because of (4.24) it is enough to compute $E^{\prime 2}\left(\widetilde{G}_{A^{o}}(a, b)\right)$ when $a$ is large.

Let $a>3$ (and thus $a>b$ ). Evidently ${E^{\prime}}^{2}\left(G_{A^{\circ}}(a, b)\right)$ has two non-zero rows, for $q=0$ and $q=b$, and therefore among differentials $d_{[p, q]}^{r}, r \geq 2$, all but those for $r=b+1$, $q=0, b<p \leq a$ are equal to zero.

On the other hand total homologies of $\widetilde{G}_{A^{\circ}}(a, b)$ are isomorphic to total homologies of $G_{A^{\circ}}(a, b)$ and their description is given in Corollary 4.5. It follows from Corollary 4.5 that

$$
\sum_{p+q=n}{E^{\prime}}_{[p, q]}^{\infty}\left(\widetilde{G}_{A^{o}}(a, b)\right)=0 \text { for } n<a, \text { and } \sum_{p+q=a}{E^{\prime}}_{[p, q]}^{\infty}\left(\widetilde{G}_{A^{o}}(a, b)\right)=\Lambda_{b}^{+} .
$$

All this forces us to conclude that the differential $d_{[p, 0]}^{(b+1)}, b<p \leq a$, determines isomorphism

$$
\left.H_{a-p}(R(a, b))={E^{\prime}}_{[p, 0]}^{b+1}\left(\widetilde{G}_{A^{o}}(a, b)\right) \longrightarrow{E^{\prime}[p-b-1, b]}_{\left(\sigma_{A^{o}}\right.}(a, b)\right)=\Lambda_{a+b-p+1}^{+} .
$$

This proves the lemma.

It is important to notice that the above morphism $d_{[p, 0]}^{(b+1)}$ is induced by $\nabla$ hence it diminishes the degree of $x$ 's by 1 . Therefore the space ${E^{\prime}}_{[p, 0]}^{b+1}\left(\widetilde{G}_{A^{o}}(a, b)\right)$ above is represented by elements of $x$-degree 1 because elements in ${E^{\prime}}_{[p-b-1, b]}^{b+1}\left(\widetilde{G}_{A^{o}}(a, b)\right)$ have $x$-degree zero.

¿From (4.24) and (4.25) we get

$$
E_{[p, 0]}^{\prime 2}\left(\widetilde{G}_{A^{o}}(a, b)\right)=H_{a-p}(R(a, b))=\Lambda^{a+b-p+1}(\text { in } x \text {-degree } 1) .
$$

To continue proving Proposition 4.6, we return to our previous data $0 \leq a \leq b \leq 3$ and conclude that if $0 \leq n \leq a$ then

$$
\sum_{m} H^{m, n}\left(\widetilde{G}_{A^{o}}(a, b)\right) \simeq{E^{\prime}}_{[n, 0]}^{\infty}\left(\widetilde{G}_{A^{o}}(a, b)\right) \simeq{E^{\prime}}_{[n, 0]}^{2}\left(\widetilde{G}_{A^{o}}(a, b)\right) \simeq \Lambda^{a+b-n+1}(\text { in } x \text {-degree } 1),
$$

hence $H\left(G_{A^{o}}(a, b)\right)^{1, n} \simeq \Lambda^{a+b-n+1}$, and $H\left(G_{A^{o}}(a, b)\right)^{m, n}=0$ for $m \neq 1$. With this we have got the rest of the statement for $X=A^{o}$.

Similarly for $X=B$ we define a " $\partial_{ \pm}$-free version", a bicomplex

$$
\left(\widetilde{G}_{B}(a, b)\right)_{[p, q]}=\left\{\begin{array}{cc}
\Lambda_{a-p}^{+} \Lambda_{b-q}^{-}[x] & \text { for } p \leq 0, q \leq 0, \\
0 & \text { overwise }
\end{array}\right.
$$

with the differentials $\mathrm{d}^{\prime}=\Delta^{+}, \mathrm{d}^{\prime \prime}=\Delta^{-}$, and looking at (4.6) we see that there is a natural isomorphism of bicomplexes $\alpha: G_{B}(a, b) \rightarrow \widetilde{G}_{B}(a, b)$.

Let

$$
\left(G_{A^{\prime}}(a, b)\right)_{[p, q]}=\left\{\begin{array}{cc}
\Lambda_{a-1}^{+} \Lambda_{b-1}^{-}[x] & \text { for } p=0, q=0, \\
0 & \text { overwise. }
\end{array}\right.
$$


It is clear that $\nabla_{2}$ maps $G_{A^{\prime}}(a, b)$ into $G_{B}(a, b)$ having $G_{B^{\circ}}(a, b)$ as the cokernel, and that $\alpha$ induces an isomorphism between $G_{B^{\circ}}(a, b)$ and the cokernel of the morphism $\Delta^{+} \Delta^{-}$from $G_{A^{\prime}}(a, b)$ into $\widetilde{G}_{B}(a, b)$, the latter cokernel we will denote by $\widetilde{G}_{B^{\circ}}(a, b)$.

Notice that for $a=b=0$ we have $G_{A^{\prime}}(0,0)=0$, so $G_{B}(0,0)=G_{B^{\circ}}(0,0)$ and it is easy to check that the proof of Proposition 4.3 works and provides the result. Let us suppose $b>0$.

Again we represent $\widetilde{G}_{B}(a, b)$ by the following diagram:

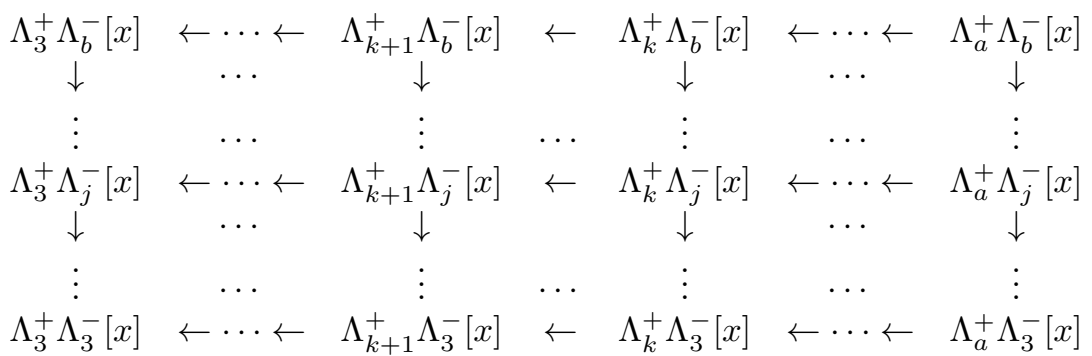

where the row (resp. column) maps are the De Rham differentials $\Delta^{+}\left(\right.$resp. $\Delta^{-}$) and the upper-right corner corresponds to $[p, q]=[0,0]$ so the diagram is situatied in the third quarter. For $\widetilde{G}_{B^{o}}(a, b)$ we are to change the upper-right corner to $\operatorname{Coker}\left(\Delta^{+} \Delta^{-}\right)$.

To compute the ${E^{\prime}}^{1}$-term of the spectral sequence we consider the column complexes and evidently they have non-zero homology only at the upper $(q=0)$ row. Having in mind that $b>0$ one easily checks that the $E^{\prime 1}$-term of the spectral sequence of the bicomplex $\widetilde{G}_{B^{\circ}}(a, b)$ is represented by the following diagram:

$$
\begin{array}{ccccccc}
\Delta^{-}\left(\Lambda_{3}^{+} \Lambda_{b-1}^{-}[x]\right) & \leftarrow \cdots \leftarrow & \Delta^{-}\left(\Lambda_{k+1}^{+} \Lambda_{b-1}^{-}[x]\right) & \leftarrow & \Delta^{-}\left(\Lambda_{k}^{+} \Lambda_{b-1}^{-}[x]\right) & \leftarrow \cdots \leftarrow \frac{\operatorname{Ker} \Delta^{-}}{\operatorname{Im} \Delta^{+} \Delta^{-}} \\
0 & \leftarrow \cdots \leftarrow & 0 & \leftarrow & 0 & \leftarrow \cdots \leftarrow & 0 \\
\vdots & \cdots & \vdots & \cdots & \vdots & \cdots & \vdots \\
0 & \leftarrow \cdots \leftarrow & 0 & \leftarrow & 0 & \leftarrow \cdots \leftarrow & 0
\end{array}
$$

Now notice that

$$
\frac{\operatorname{Ker} \Delta^{-}}{\operatorname{Im} \Delta^{+} \Delta^{-}} \simeq \frac{\Delta^{-}\left(\Lambda_{a}^{+} \Lambda_{b-1}^{-}[x]\right)}{\Delta^{+} \Delta^{-}\left(\Lambda_{a-1}^{+} \Lambda_{b-1}^{-}[x]\right)} \simeq \operatorname{Coker}\left(\Delta^{-}\left(\Lambda_{a}^{+} \Lambda_{b-1}^{-}[x]\right) \stackrel{\stackrel{\Delta}{ }^{+}}{\leftarrow} \Delta^{-}\left(\Lambda_{a-1}^{+} \Lambda_{b-1}^{-}[x]\right)\right)
$$

Now comparing the upper row with the complex $S(3, b-1)$ of Lemma 4.7 we have no difficulty determining its homology. The upper row of the diagram for ${E^{\prime}}^{2}$ becomes

$$
\Lambda^{a+b+3}, \quad \ldots, \quad \Lambda^{k+b+1}, \quad \Lambda^{k+b}, \quad \ldots, \quad \Lambda^{a+b} .
$$

There are no more non-zero differentials, and we obtain the result in this case.

The reasoning for $X=D$ goes along the same lines and the situation is in some sense dual to this in the case $X=A$. We will stress the main points leaving it to the reader to fill in details. 
The diagram representing $\widetilde{G}_{D^{o}}(a, b)$ is the following (we skip the evident definition of $\widetilde{G}_{\left.D^{o}(a, b)\right) \text { : }}$

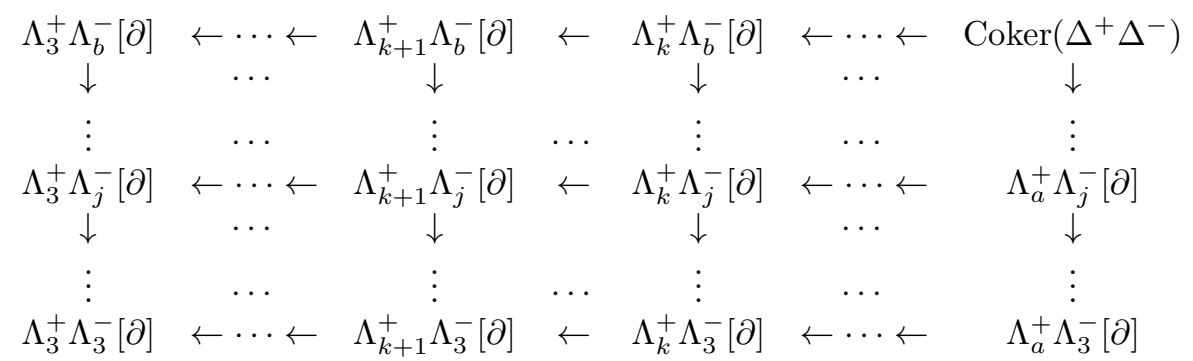

where the row (resp. column) maps $\Delta^{+}$(resp. $\Delta^{-}$) are Koszul differentials, the upper-right corner corresponds to $[p, q]=[0,0]$, the diagram is situatied in the third quarter and its other corners are $[0, b-3],[a-3,0],[a-3, b-3]$.

Calculating homologies of the vertical complexes we get (provided $b>0$ ) the following diagram:

$$
\begin{array}{ccccccc}
\Delta^{-}\left(\Lambda_{3}^{+} \Lambda_{b-1}^{-}[\partial]\right) & \leftarrow \cdots \leftarrow & \Delta^{-}\left(\Lambda_{k+1}^{+} \Lambda_{b-1}^{-}[\partial]\right) & \leftarrow & \Delta^{-}\left(\Lambda_{k}^{+} \Lambda_{b-1}^{-}[\partial]\right) & \leftarrow \cdots \leftarrow & \text { Ker } \Delta^{-} \\
0 & \leftarrow \cdots \leftarrow & 0 & \leftarrow & 0 & \leftarrow \cdots \leftarrow & 0 \\
\vdots & \cdots & \vdots & \cdots & \vdots & \cdots & \vdots \\
0 & \leftarrow \cdots \leftarrow & 0 & \leftarrow & 0 & \leftarrow \cdots \leftarrow & 0 \\
\Lambda_{3}^{+} \Lambda_{3}^{-} & \leftarrow \cdots \leftarrow & \Lambda_{k+1}^{+} \Lambda_{3}^{-} & \leftarrow & \Lambda_{k}^{+} \Lambda_{3}^{-} & \leftarrow \cdots \leftarrow & \Lambda_{a}^{+} \Lambda_{3}^{-}
\end{array}
$$

Clearly the term at the upper-right corner is isomorpic to

$$
\frac{\operatorname{Ker} \Delta^{-}}{\operatorname{Im} \Delta^{+} \Delta^{-}} \simeq \frac{\operatorname{Im} \Delta^{-}}{\operatorname{Im} \Delta^{+} \Delta^{-}} \simeq \operatorname{Coker}\left(\Delta^{-}\left(\Lambda_{a}^{+} \Lambda_{b-1}^{-}[\partial]\right) \stackrel{\Delta^{+}}{\leftarrow} \Delta^{-}\left(\Lambda_{a-1}^{+} \Lambda_{b-1}^{-}[\partial]\right)\right)
$$

and we evaluate the homologies of the complex in the upper row by the following lemma (which is analogous to Lemma 4.7).

Lemma 4.8. Let $b>0$ and $T(a, b)$ be the complex

$$
\Delta^{-}\left(\Lambda_{3}^{+} \Lambda_{b-1}^{-}[\partial]\right) \quad \stackrel{\Delta^{+}}{\leftarrow} \quad \Delta^{-}\left(\Lambda_{2}^{+} \Lambda_{b-1}^{-}[\partial]\right) \quad \stackrel{\Delta^{+}}{\leftarrow} \ldots \stackrel{\Delta^{+}}{\leftarrow} \quad \Delta^{-}\left(\Lambda_{k}^{+} \Lambda_{b-1}^{-}[\partial]\right) \quad \stackrel{\Delta^{+}}{\leftarrow} \ldots \stackrel{\Delta^{+}}{\leftarrow} T(a, b)_{a},
$$

where $T(a, b)_{a}=$ Coker $\left(\Delta^{-}\left(\Lambda_{a+1}^{+} \Lambda_{b-1}^{-}[\partial]\right) \stackrel{\Delta^{+}}{\leftarrow} \Delta^{-}\left(\Lambda_{a}^{+} \Lambda_{b-1}^{-}[\partial]\right)\right)$.

Then homologies of $T(a, b)$ are isomorphic to:

$$
\Lambda^{b-1}, \quad \Lambda^{b-2}, \quad \ldots, \quad \Lambda^{b-k}, \quad \ldots, \quad \Lambda^{b-1+a-3},
$$

and are represented by the elements linear in $\partial_{i}, i=1,2,3$.

We leave it to the reader to prove the lemma by the same trick of changing $a$ and comparing the first and the second spectral sequences of the bicomplex in question.

The condition $0 \leq b \leq a$ implies that no futher differentials of the spectral sequence are non-zero. Thus ${E^{\prime 2}}^{2}={E^{\prime}}^{\infty}$ and this gives us the result in the $D$-case. 
For $C$-case the diagram representing $E^{\prime 1}\left(\widetilde{G}_{C^{o}}(a, b)\right)$ situated again in the first quarter and it is

$$
\begin{array}{ccccccc}
0 & \leftarrow \cdots \leftarrow & 0 & \leftarrow & 0 & \leftarrow \cdots \leftarrow & 0 \\
\vdots & \cdots & \vdots & \cdots & \vdots & \cdots & \vdots \\
0 & \leftarrow \cdots \leftarrow & 0 & \leftarrow & 0 & \leftarrow \cdots \leftarrow & 0 \\
\frac{\operatorname{Ker}\left(\Delta^{+} \Delta^{-}\right)}{\operatorname{Im} \Delta^{-}} & \leftarrow \cdots \leftarrow & \Delta^{-}\left(\Lambda_{k+1}^{+} \Lambda_{b}^{-}[\partial]\right) & \leftarrow & \Delta^{-}\left(\Lambda_{k}^{+} \Lambda_{b}^{-}[\partial]\right) & \leftarrow \cdots \leftarrow & \Delta^{-}\left(\Lambda_{0}^{+} \Lambda_{b}^{-}[\partial]\right),
\end{array}
$$

Clearly the term at the lower-left corner is isomorpic to

$$
\frac{\operatorname{Ker}\left(\Delta^{+} \Delta^{-}\right)}{\operatorname{Im} \Delta^{-}} \simeq \operatorname{Ker}\left(\Delta^{-}\left(\Lambda_{a}^{+} \Lambda_{b-1}^{-}[\partial]\right) \stackrel{\Delta^{+}}{\leftarrow} \Delta^{-}\left(\Lambda_{a-1}^{+} \Lambda_{b-1}^{-}[\partial]\right)\right)
$$

and the values of the horisontal homologies could be found from those of $T(0, b)$ given by Lemma 4.8. This closes the last case and completes the proof of Propositon 4.6 .

Corollary 4.9. By interchanging $a, b$ in Proposition 4.0 we get a valid statement as well.

We are to change rows into columns and use the other spectral sequence of a bicomplex.

Given $n \in \mathbb{Z}, y \in \mathbb{C}$, let $P(n, y)$ be an irreducible $s \ell(2) \oplus g \ell(1)$-module with highest weight $(n, y)$ when $n \geq 0$ and $P(n, y)=0$ when $n<0$.

Theorem 4.10. There are the following isomorphisms of $\mathrm{g}_{0}$-modules (sums below run over $0 \leq i \leq 3)$ :

(a)

$$
H^{m, n}\left(G_{A^{\circ}}\right)=\left\{\begin{array}{cl}
\sum \Lambda^{i} \otimes P\left(n-i,-\frac{1}{3} i-n\right) & \text { for } m=0, n \geq 0, \\
\sum \Lambda^{i} \otimes P\left(i-n-1,-\frac{1}{3} i-n+1\right) & \text { for } m=1,0 \leq n \leq 3, \\
0 & \text { otherwise. }
\end{array}\right.
$$

$$
H^{m, n}\left(G_{B^{o}}\right)=\left\{\begin{array}{cl}
\sum \Lambda^{i} \otimes P\left(-n+i,-\frac{1}{3} i-n+2\right) & \text { for } m=0, n \leq 0, \\
0 & \text { otherwise. }
\end{array}\right.
$$

(c)

$$
H^{m, n}\left(G_{C^{o}}\right)=\left\{\begin{array}{cl}
\sum \Lambda^{i} \otimes P\left(n+3-i,-\frac{1}{3} i-n-3\right) & \text { for } m=0, n \geq 0, \\
0 & \text { otherwise. }
\end{array}\right.
$$

$$
H^{m, n}\left(G_{D^{o}}\right)=\left\{\begin{array}{cl}
\sum \Lambda^{i} \otimes P\left(-n-3+i,-\frac{1}{3} i-n-1\right) & \text { for } m=0, n \leq 0 \\
\sum \Lambda^{i} \otimes P\left(n-i+2,-\frac{1}{3} i-n-2\right) & \text { for } m=-1,-2 \leq n \leq 0 \\
0 & \text { otherwise. }
\end{array}\right.
$$


Proof. To prove the statements we use the decomposition (4.7) and then collect the information about homologies for various values of $a, b$ from Propositions 4.3, 4.6 and Corollaries 4.4, 4.5, 4.9, having in mind that $h_{3}$ acts on $G_{X^{o}}(a, b)$ as multiplication by $a-b$.

The action of $Y$ has to be computed too. It is easy to compute it for $E^{2}$ by choosing a representative for a homology class. Then it descends to $E^{\infty}$ and because of the convergence of the spectral sequences we immediately determine the action on homologies. We leave the details to the reader.
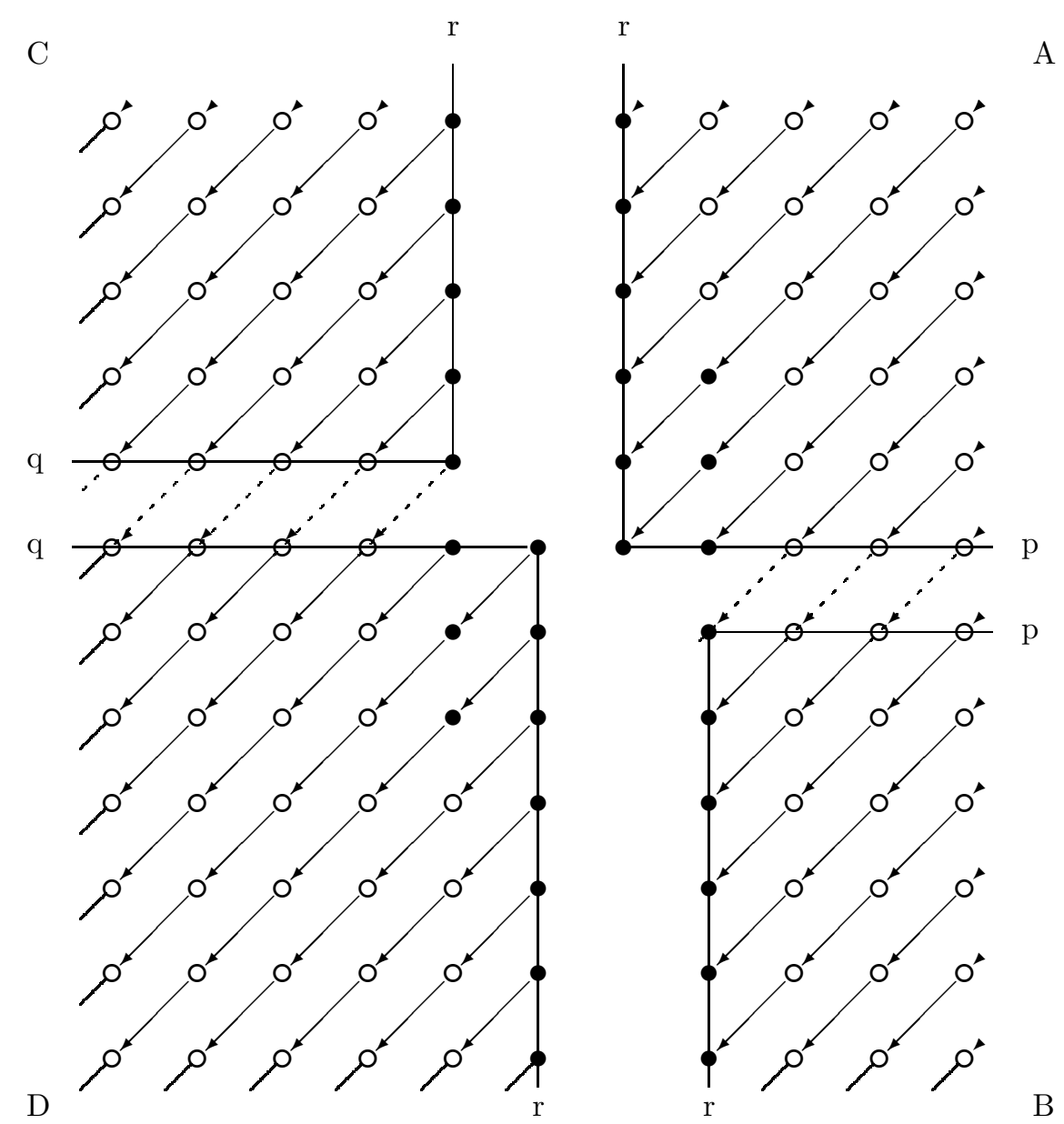

Figure 2.

We see that Theorem 4.10 provides us with the information about the homologies of the complexes $\operatorname{Gr} M_{X^{\circ}}$ (and hence $M_{X}$ for $X=A B, C D$ ). In the following Figure 2 this information is presented graphically. The white circles mark those places $(m, n)$ where the homologies $H^{m, n}\left(\operatorname{Gr} M_{X^{o}}\right)$ are zero, and black nodes mark those positions where they may be non-zero.

Proof of Proposition 3.3. If either $m \geq 2$ or $m=1, n \neq 0,1,2$, then, by Theorem 4.10, $H^{m, n}\left(G_{X}\right)=0$ for $X=A^{o}, B^{o}$. Equations 4.3 show that $H^{m, n}\left(G_{A B}\right)=0$ under the same 
conditions on $m, n$, and hence $H^{m, n}\left(M_{A B}\right)=0$ by Proposition 3.2. In the same way using Theorem 4.10 and equations (4.4) we prove the second part of the proposition.

For a linear map $\nabla: M^{\prime} \rightarrow M^{\prime \prime}$ we shall use the notations:

$$
\begin{array}{ll}
\operatorname{Ker}\left(\nabla \mid M^{\prime}\right) & =\operatorname{Ker}\left(\nabla: M^{\prime} \rightarrow M^{\prime \prime}\right), \\
\operatorname{Im}\left(\nabla \mid M^{\prime \prime}\right) & =\operatorname{Im}\left(\nabla: M^{\prime} \rightarrow M^{\prime \prime}\right)=\operatorname{Im}\left(\left.\nabla\right|_{M^{\prime}}\right), \\
\operatorname{Coker}\left(\nabla \mid M^{\prime \prime}\right) & =\operatorname{Coker}\left(\nabla: M^{\prime} \rightarrow M^{\prime \prime}\right) .
\end{array}
$$

Proposition 4.11. Morphism $\nabla_{3}$ induces the following isomorphisms:

$$
\begin{aligned}
& \operatorname{Coker}\left(\nabla \mid M_{A}^{0, n}\right) \longrightarrow \operatorname{Ker}\left(\nabla \mid M_{C}^{0, n-3}\right), \quad n \geq 4, \\
& \operatorname{Coker}\left(\nabla \mid M_{A}^{0,3}\right) \longrightarrow \operatorname{Ker}\left(\nabla_{2} \mid M_{C}^{0,0}\right), \\
& \operatorname{Coker}\left(\nabla \mid M_{B}^{0, n}\right) \longrightarrow \operatorname{Ker}\left(\nabla \mid M_{D}^{0, n-3}\right), \quad n<0, \\
& \operatorname{Coker}\left(\nabla_{2} \mid M_{B}^{0,0}\right) \longrightarrow \operatorname{Ker}\left(\nabla \mid M_{D}^{0,-3}\right) .
\end{aligned}
$$

Proof. To prove that a morphism of filtered modules with a differential induces an isomorphism on homologies it is sufficient to show that it induces an isomorphism of the initial terms of their spectral sequences and the initial terms are of the form $H\left(\operatorname{Gr} M_{X}\right)$.

Because of (3.6) and (4.1), (4.2) it is enough to establish that $\nabla_{3}$ induces isomorphisms:

$$
H^{0, n}\left(G_{A^{\circ}}\right) \rightarrow H^{0, n-3}\left(G_{C^{\circ}}\right) \text { for } n \geq 3, \quad H^{0, n}\left(G_{B^{\circ}}\right) \rightarrow H^{0, n-3}\left(G_{D^{\circ}}\right) \text { for } n \leq 0 .
$$

Theorem 4.10 shows that the corresponding homologies are indeed isomorphic $g_{0}$-modules, hence we should check only that $\nabla_{3}$ maps each of the highest weight vectors in $H^{0, n}\left(G_{A^{\circ}}\right)$ (resp. $H^{0, n}\left(G_{B^{\circ}}\right)$ ) to a non-zero element of $H^{0, n-3}\left(G_{C^{o}}\right)$ (resp. $H^{0, n-3}\left(G_{D^{o}}\right)$ ). The representatives for highest weight vectors are

$$
z_{+}^{n}, d_{1}^{+} z_{+}^{n-1} z_{-}, d_{1}^{+} d_{2}^{+} z_{+}^{n-2} z_{-}^{2}, d_{1}^{+} d_{2}^{+} d_{3}^{+} z_{+}^{n-3}, z_{-}^{3}
$$

for $H^{0, n}\left(G_{A^{o}}\right)$, and

$$
\partial_{-}^{-n}, d_{1}^{+} \partial_{-}^{-n-1} \partial_{+}, d_{1}^{+} d_{2}^{+} \partial_{-}^{-n-2} \partial_{+}^{2}, d_{1}^{+} d_{2}^{+} d_{3}^{+} \partial_{-}^{-n-3} \partial_{+}^{3}
$$

for $H^{0, n}\left(G_{B^{\circ}}\right)$. Now it is immediate to see that the images are non-zero.

Proposition 4.12. Morphisms $\nabla_{4}^{\prime}$, $\nabla_{4}^{\prime \prime}$ induce isomorphisms:

$$
\begin{aligned}
& \nabla_{4}^{\prime}: \quad \operatorname{Coker}\left(\nabla \mid M_{A}^{0,2}\right) \longrightarrow \operatorname{Ker}\left(\nabla \mid M_{D}^{-1,0}\right), \\
& \nabla_{4}^{\prime \prime}: \quad \operatorname{Coker}\left(\nabla \mid M_{A}^{1,0}\right) \longrightarrow \operatorname{Ker}\left(\nabla \mid M_{D}^{0,-2}\right) .
\end{aligned}
$$

Proof. Again it is sufficient to establish that the maps induce isomorphisms of the initial terms of the spectral sequences that are isomorphic $\mathrm{g}_{0}$-modules because of Theorem 4.10 . Thus again we have to check the images of the highest vectors of $H^{0,2}\left(G_{A^{\circ}}\right)$ and $H^{1,0}\left(G_{A^{\circ}}\right)$.

For $H^{0,2}\left(G_{A^{\circ}}\right)$ the representatives of the highest weight vectors are

$$
z_{+}^{2}, d_{1}^{+} z_{+}^{1} z_{-}, d_{1}^{+} d_{2}^{+} z_{-}^{2},
$$

and their images

$$
d_{123}^{+} \Delta^{-}, d_{1}^{+} d_{1}^{-} d_{23}^{+} \Delta^{-}, d_{12}^{+}\left(d_{12}^{-} d_{3}^{+}+d_{1}^{-} d_{2}^{+} d_{3}^{-}\right) \Delta^{-},
$$

are indeed non-zero in $H^{0,-2}\left(G_{D^{o}}\right)$. 
Similarly for $H^{1,0}\left(G_{A^{\circ}}\right)$ the representatives are

$$
x_{1}, d_{1}^{+} x_{2}-d_{2}^{+} x_{1}, d_{1}^{+} d_{2}^{+} x_{3}+d_{2}^{+} d_{3}^{+} x_{1}+d_{3}^{+} d_{2}^{+} x_{1} .
$$

We use (2.17) and calculate in $G_{D}=\Lambda\left(\mathrm{g}_{-1}\right) \otimes V_{D}$. We leave to the reader to make the calculations and see that the results are indeed non-zero.

\section{Homology of $M_{X}$ and secondary singular vectors.}

We return to the spectral sequence for $M_{X}$, decribed in Section 3. We are particulary interested in eight cases when $X=A$ or $D$, which have been left unfinished in the previous section. The description of the initial term $E^{0}=H\left(\operatorname{Gr} M_{X}\right)$ of the spectral sequences in these cases follows from Theorem 4.10 and was presented in Figure 2. In this section we compute the subsequent terms of these spectral sequences and hence the homology.

Theorem 5.1. There are the following isomorphisms of $E(3,6)$-modules:

(i) $H^{0,0}\left(M_{A}\right)=\mathbb{C}, \quad H^{1,2}\left(M_{A}\right)=0$,

(ii) $H^{0,1}\left(M_{A}\right) \simeq H^{1,1}\left(M_{A}\right) \simeq I(0,0 ; 1 ;-1)$,

(iii) $H^{0,0}\left(M_{D}\right)=0, \quad H^{-1,-2}\left(M_{D}\right)=\mathbb{C}$,

(iv) $H^{0,-1}\left(M_{D}\right) \simeq H^{-1,-1}\left(M_{D}\right) \simeq I(0,0 ; 1 ;-1)$.

In the following we denote by $\nabla$ any of the morphisms $\nabla, \nabla_{1}, \ldots, \nabla_{6}$ (see Figure 1) assuming that it is clear from the context which map is considered.

¿From now on we combine the differential $E(3,6)$-modules $M_{A B}$ and $M_{C D}$ in one differential $E(3,6)$-module $\mathbf{M}$ represented by Figure 3 . The $E(3,6)$-module $\mathbf{M}$ equals to the direct sum

$$
\mathbf{M}=\sum_{(m, n) \neq(0,0)} M_{A}^{m, n} \oplus M_{B} \oplus M_{C} \oplus \sum_{(m, n) \neq(0,0)} M_{D}^{m, n},
$$

and the maps are the same as in Figure 1 except for the map $\widetilde{\nabla}: M_{A}^{1,1} \longrightarrow M_{D}^{-1,-1}$ which is defined to be equal to the composition:

$$
M_{A}^{1,1} \stackrel{\nabla}{\longrightarrow} M_{A}^{0,0} \simeq M_{D}^{0,0} \stackrel{\nabla}{\longrightarrow} M_{D}^{-1,-1} .
$$

White nodes in Figure 3 mark the positions where the kernel of the outcoming map equals to the image of the incoming one (i.e. the corresponding homology of $\mathbf{M}$ is zero). The black marks denote the places with non-zero homology. Namely, the star refers to the trivial module $\mathbb{C}$, the diamond to $I(0,0 ; 1 ;-1)$ and the spade to the module $P=I(0,0 ; 1 ;-1) \oplus \mathbb{C}$.

The above description of the homologies follows from Theorem 5.1, Propositions 3.3, 4.11, 4.12, and 5.27. Saying that $P$ is a direct sum we have taken into account that an extension

$$
0 \longrightarrow \mathbb{C} \longrightarrow P \longrightarrow I(0,0 ; 1 ;-1) \longrightarrow 0
$$

is necessary a direct sum, because all eigenvalues of $Y$ on $I(0,0 ; 1 ;-1)$ are strictly negative, but $Y$ acts on $\mathbb{C}$ with eigenvalue zero.

Corollary 5.2. The complex $\mathbf{M}$ decomposes in a direct sum of $\mathbb{Z}$-graded complexes, that are infinite in both directions and consist of generalized Verma modules $M_{X}^{m, n}$. Homologies of these complexes are all zero except those that appear at terms $M_{A}^{1,1} \simeq M\left(1,0 ; 1 ;-\frac{2}{3}\right)$, $M_{D}^{-1,-1} \simeq M\left(0,1 ; 1 ; \frac{2}{3}\right)$ and $M_{D}^{-1,-2} \simeq M\left(0,1 ; 2 ; \frac{5}{3}\right)$.

We use below the notations (4.27). 


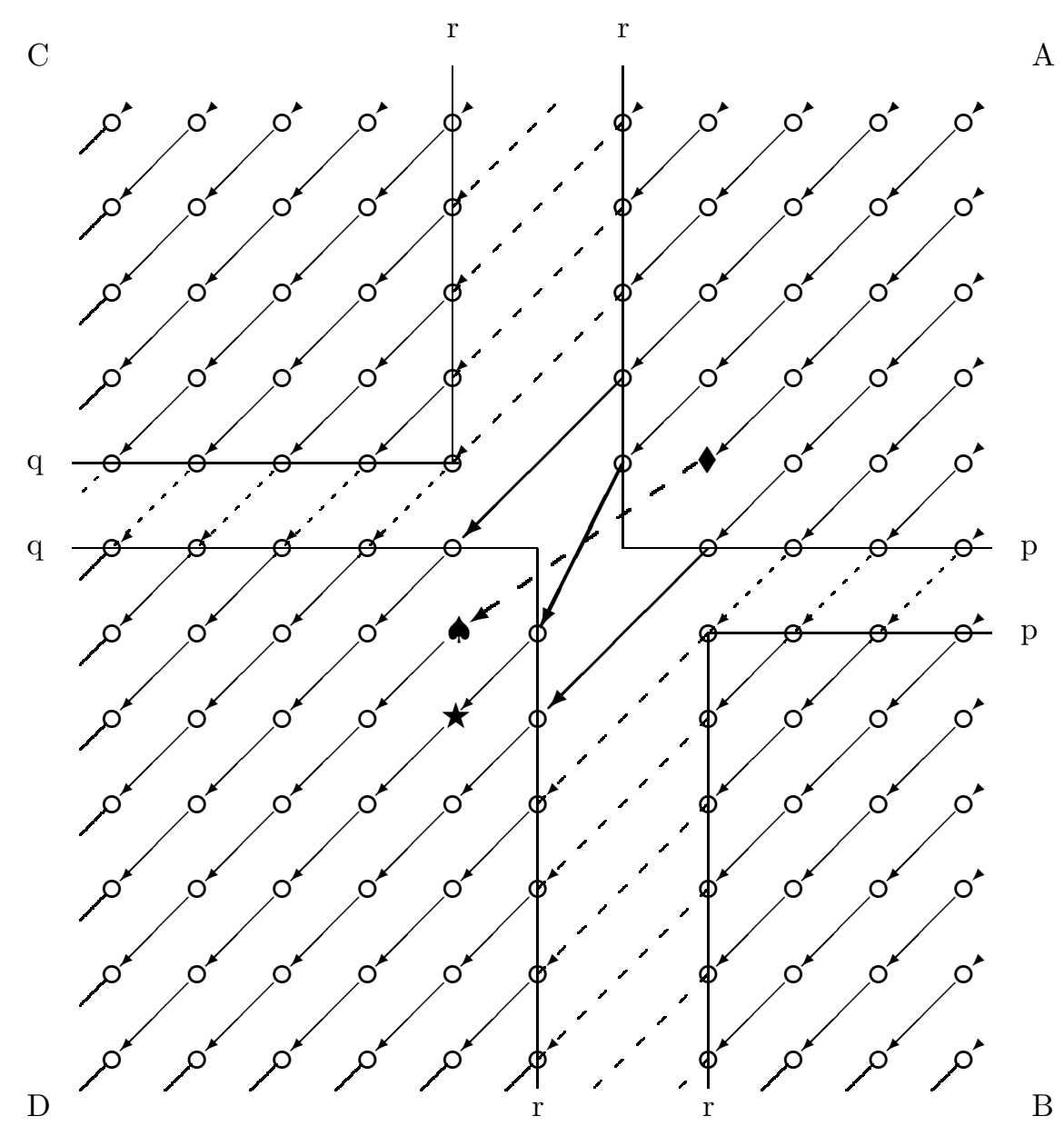

FiguRE 3 .

Corollary 5.3. For the $E(3,6)$-modules $M\left(p, q ; r ; y_{X}\right)$ corresponding to the white nodes of Figure 目 (and for $M(0,0 ; 0 ; 0)) \operatorname{Ker} \nabla=\operatorname{Im} \nabla$ is a unique non-trivial submodule, it is isomorphic to the irreducible quotient of the previous module of the complex, and the quotient by it is isomorphic to $I\left(p, q ; r ; y_{X}\right)$. Equivalently, $\operatorname{Im}\left(\nabla \mid M_{X}^{m, n}\right)$ is a unique non-trivial submodule of $M_{X}^{m, n}$ except when $M_{X}^{m, n}=M_{A}^{1,1}, M_{D}^{-1,-1}, M_{D}^{-1,-2}$.

Thus, all degenerate generalized Verma modules $M\left(p, q ; r ; y_{X}\right)$, except for $M\left(1,0 ; 1 ; y_{A}\right)$, $M\left(0,1 ; 1 ; y_{D}\right)$ and $M\left(0,1 ; 2 ; y_{D}\right)$, have a unique non-trivial submodule.

Corollary 5.4. The only nontrivial submodules of the excluded above modules $M\left(1,0 ; 1 ; y_{A}\right)$, $M\left(0,1 ; 2 ; y_{D}\right)$ and $M\left(0,1 ; 1 ; y_{D}\right)$ are the following

(i) $\operatorname{Im} \nabla \subset \operatorname{Ker} \nabla$ for $M\left(1,0 ; 1 ; y_{A}\right)$, with subquotients respectively isomorphic to $I\left(2,0 ; 2 ; y_{A}\right), I\left(0,0 ; 1 ; y_{A}\right), I\left(1,0 ; 1 ; y_{A}\right)$,

(ii) $\operatorname{Im} \nabla \subset \operatorname{Ker} \nabla$ for $M\left(0,1 ; 2 ; y_{D}\right)$, with subquotients $I\left(0,0 ; 1 ; y_{D}\right)$, $\mathbb{C}, I\left(0,1 ; 2 ; y_{D}\right)$,

(iii) $S_{i}, i=0,1,2,3$ for $M\left(0,1 ; 1 ; y_{D}\right)$, where

$$
S_{0}=\operatorname{Im} \widetilde{\nabla}, \quad S_{1}=\operatorname{Im} \nabla, \quad S_{2}=U(L) q_{+}+S_{0}, \quad S_{3}=\operatorname{Ker} \nabla,
$$




$$
\begin{gathered}
\text { and } q_{+} \text {is defined before Lemma 5.24. One has: } S_{0}=S_{1} \cap S_{2}, S_{3}=S_{1}+S_{2}, \\
S_{0} \simeq I\left(1,0 ; 1 ; y_{A}\right), \quad S_{3} / S_{0} \simeq \mathbb{C} \oplus I\left(0,0 ; 1 ; y_{A}\right), \quad M\left(0,1 ; 1 ; y_{D}\right) / S_{3} \simeq I\left(0,1 ; 1 ; y_{D}\right) .
\end{gathered}
$$

The results of Corollary 5.4 will be established while proving Theorem 5.1. To prove Corollary 5.3 we need the following lemma.

Lemma 5.5. Let $M^{\prime \prime} \stackrel{\nabla^{\prime \prime}}{\leftarrow} M^{\prime} \stackrel{\nabla^{\prime}}{\leftarrow} M$ be an exact sequence of highest weight modules over $L$ with highest weight vectors $m^{\prime \prime}, m^{\prime}$ and $m$ respectively. Suppose that $\nabla^{\prime}(m)$ is a unique (up to a constant factor) non-trivial singular vector of $M^{\prime}$. Then

$$
\operatorname{Im} \nabla^{\prime}=\operatorname{Ker} \nabla^{\prime \prime}
$$

is an irreducible $L$-submodule of $M^{\prime}$.

Suppose also that $\nabla^{\prime \prime}\left(m^{\prime}\right)$ is a unique (up to a constant factor) non-trivial singular vector of $M^{\prime \prime}$. Then

$$
\operatorname{Im} \nabla^{\prime \prime} \simeq \operatorname{Coker} \nabla^{\prime}=M^{\prime} / \operatorname{Im} \nabla^{\prime}
$$

is irreducible too, thus $\operatorname{Im} \nabla^{\prime}$ is a unique non-trivial submodule in $M^{\prime}$.

Proof. Due to exactness, the module $\operatorname{Ker} \nabla^{\prime \prime}$ is a quotient of the highest weight module $M^{\prime}$. Hence, having by the conditions only trivial singular vectors, it is irreducible.

Furthermore, by exactness we have the isomorphism of Coker $\nabla^{\prime}$ and $\operatorname{Im} \nabla^{\prime \prime}$. Due to uniqueness of a non-trivial singular vector in $M^{\prime \prime}$, we conclude, as above, that $\operatorname{Im} \nabla^{\prime \prime}$ is irreducible.

Proof of Corollary 5.3. We use Corollary 5.2 to provide exact sequences and Corollary 2.11 to ensure the uniqueness of non-trivial singular vectors.

We also get the fact that $\operatorname{Im}\left(\nabla \mid M_{A}^{0,0}\right)$ is irreducible applying the first part of Lemma 5.5 to the sequence $\mathbb{C} \leftarrow M_{A}^{0,0} \leftarrow M_{A}^{1,1}$. Then $M_{A}^{0,0} / \operatorname{Im}\left(\nabla \mid M_{A}^{0,0}\right)=H^{0,0}\left(M_{A}\right) \simeq \mathbb{C}$ by Theorem 5.1, so we get the statement for $M_{A}^{0,0}=M_{D}^{0,0}$.

To prove Theorem 5.1 we are to compute the differentials in the spectral sequence $\left\{E^{i}\left(M_{X}\right), \nabla^{(i)}\right\}$ for the homology $H\left(M_{X}\right), X=A, D$, and for this we need to consider representatives of various cycles.

Definition 5.6. We say that an element $s \in M_{X}^{m, n}$ represents the highest weight vector of $H^{m, n}\left(G_{X}\right)$ if

(1) $s$ is a non-zero $\mathrm{g}_{0}$-highest weight vector (in $M_{X}^{m, n}$ ),

(2) the image $[s]$ of $s$ in $\operatorname{Gr} M_{X}$ is a cycle,

(3) $[s]$ belongs to $G_{X} \subset \operatorname{Gr} M_{X}$.

It follows that the homology class of $[s]$ in $H^{m, n}\left(G_{X}\right)$ is a $\mathrm{g}_{0}$-highest weight vector.

We say that $s_{0}, \ldots, s_{k} \in M_{X}^{m, n}$ represent a basis of $H^{m, n}\left(G_{X}\right)$ if

$\left(1^{\prime}\right)$ the span of $s_{0}, \ldots, s_{k}$ is a $\mathrm{g}_{0}$-submodule in $M_{X}^{m, n}$,

$\left(2^{\prime}\right)$ all $\left[s_{i}\right]$ are cycles and belong to $G_{X} \subset \operatorname{Gr} M_{X}$,

$\left(3^{\prime}\right)\left\{\left[s_{i}\right]\right\}$ give us a basis for $H^{m, n}\left(G_{X}\right)$.

Definition 5.7. We say that $s \in M_{X}^{m, n}$ represents a singular vector of $H^{m, n}\left(M_{X}\right)$ if

(1) $s$ is a non-zero $\mathrm{g}_{0}$-highest weight vector,

(2) $s$ is a cycle in $M_{X}$,

(3) $e_{0} s \in \operatorname{Im} \nabla, e_{0}^{\prime} s \in \operatorname{Im} \nabla$. 
Whenever $c_{1}, c_{2}$ are cycles, the notation $c_{1} \equiv c_{2}$ means $c_{1}$ and $c_{2}$ belong to the same homology class.

Let us notice that due to Remark 3.1 the terms $E^{i}\left(M_{X}\right)^{m, n}$ are $W$-modules and clearly they are $\mathrm{g}_{0}$-modules as well. Thus we may consider the action on these terms of the following subalgebra $\mathcal{W}$ of $E(3,6)$

$$
\mathcal{W}=W+\mathrm{g}_{0} \simeq W \oplus s \ell(2) .
$$

Given a go-module $V$ with the trivial action of $L_{1}^{\mathcal{W}}=L_{1}^{W}$ we get an isomorphism:

$$
\mathrm{S}\left(\mathrm{g}_{-2}\right) \otimes_{\mathbb{C}} V \simeq \operatorname{Ind}_{L_{0}^{\mathcal{W}}}^{\mathcal{W}}(V)
$$

We conclude that (3.5), (3.6) lead us to the following isomorphisms of $\mathcal{W}$-modules:

$$
\operatorname{Gr} M_{X} \simeq \operatorname{Ind}_{L_{0}^{\mathcal{W}}}^{\mathcal{W}}\left(\Lambda\left(\mathrm{g}_{-1}\right) \otimes V_{X}\right), \quad H^{m, n}\left(\operatorname{Gr} M_{X}\right) \simeq \operatorname{Ind}_{L_{0}^{w v}}^{\mathcal{W}}\left(H^{m, n}\left(G_{X}\right)\right)
$$

Therefore we can speak of $\mathcal{W}$-singular vectors in $\operatorname{Gr} M_{X}$ or in $H^{m, n}\left(\operatorname{Gr} M_{X}\right)$ (see [KR1] for general properties of singular vectors).

Remark 5.8. Formulae (1.3) and (1.4) show that the central element $\sum x_{i} \partial_{i} \in g \ell(3) \subset W_{3}$ corresponds to $\frac{3}{2} Y \in g \ell(3) \subset W \subset \mathcal{W}$.

Because of Remark 5.8 we notice that the following $g \ell(3) \oplus s \ell(2)$-modules are isomorphic to each other

$$
V=F\left(0,1 ; 0 ;-\frac{2}{3}\right) \simeq \Lambda^{2} \otimes P\left(0,-\frac{2}{3}\right) \simeq \mathrm{g}_{-2} \simeq\left(\mathbb{C}^{3}\right)^{*} \otimes \mathbb{C} \simeq V_{D}^{-1,0}=\left\langle\partial_{1}, \partial_{2}, \partial_{3}\right\rangle .
$$

Let $T^{k}=\operatorname{Ind}_{L_{0}^{\mathcal{W}}}^{\mathcal{W}}\left(\Lambda^{k} V\right)$ with $V$ defined by the isomorphisms (5.4) above. It is clear that with respect to its $W_{3}$-module structure the module $T^{k}$ is the dual to the differential forms module $\Omega_{3}^{k}$. Hence dualizing the De Rham complex $\Omega_{3}^{\bullet}$ we get an exact sequence of $\mathcal{W}$-modules:

$$
0 \leftarrow \mathbb{C} \stackrel{\sigma_{0}}{\longleftarrow} T^{0} \stackrel{\sigma_{1}}{\longleftarrow} T^{1} \stackrel{\sigma_{2}}{\longleftarrow} T^{2} \stackrel{\sigma_{3}}{\longleftarrow} T^{3} \leftarrow 0 .
$$

Denote $R=\operatorname{Ker}\left(\sigma_{1}\right)=\operatorname{Coker}\left(\sigma_{3}\right)$ and $Q=\operatorname{Ind}_{L_{0}^{\mathcal{W}}}^{\mathcal{W}}\left(\Lambda^{0} \otimes P(1,-1)\right)=\operatorname{Ind}_{L_{0}^{\mathcal{N}}}^{\mathcal{W}} V_{A}^{0,1}$.

Lemma 5.9. There are the following isomorphisms of $\mathcal{W}$-modules:

(1) $E^{0}\left(M_{A}\right)^{0,0} \simeq T^{0}, \quad E^{0}\left(M_{A}\right)^{1,1} \simeq T^{1} \oplus Q$,

(2) $E^{0}\left(M_{A}\right)^{0,1} \simeq T^{2} \oplus Q, \quad E^{0}\left(M_{A}\right)^{1,2} \simeq T^{3}$,

(3) $E^{0}\left(M_{D}\right)^{-1,-2} \simeq T^{0}, \quad E^{0}\left(M_{D}\right)^{0,-1} \simeq T^{1} \oplus Q$,

(4) $E^{0}\left(M_{D}\right)^{-1,-1} \simeq T^{2} \oplus Q, E^{0}\left(M_{D}\right)^{0,0} \simeq T^{3}$,

Statements (1) and (2) (resp. statements (3), (4) ) follow from Theorem 4.10 as soon as we take into account equations (5.3) and accomodate to the new notations.

Proposition 5.10. There are the following isomorphisms of $\mathcal{W}$-modules:

(1) $E^{1}\left(M_{A}\right)^{0,0} \simeq \mathbb{C}, \quad E^{1}\left(M_{A}\right)^{1,1} \simeq R \oplus Q$,

(2) $E^{1}\left(M_{A}\right)^{0,1} \simeq R \oplus Q, \quad E^{1}\left(M_{A}\right)^{1,2} \simeq 0$,

(3) the spectral sequence $\left\{E_{i}\left(M_{A}\right)\right\}$ degenerates at $E^{1}$, that is $E^{1}\left(M_{A}\right)^{m, n} \simeq E^{\infty}\left(M_{A}\right)^{m, n}$. 
To prove the proposition we shall calculate the differentials $\nabla^{(i)}$ starting with $\nabla^{(0)}$. We need first to find the representatives of the bases of $H^{m, n}\left(G_{A}\right) \subset E^{0}\left(M_{A}\right)$ for $(m, n)=$ $(1,2),(0,1),(1,1),(0,0)$. This is done in the following lemmae.

Let $\zeta_{i}=d_{i}^{-} z_{+}-d_{i}^{+} z_{-}$.

Lemma 5.11. The element $s=\frac{1}{3}\left(\alpha_{-} z_{+}^{2}-\alpha_{0} z_{+} z_{-}+\alpha_{+} z_{-}^{2}\right) \in M_{A}^{1,2}$, where

$$
\alpha_{ \pm}=d_{1}^{ \pm} d_{2}^{ \pm} x_{3}+d_{2}^{ \pm} d_{3}^{ \pm} x_{1}+d_{3}^{ \pm} d_{1}^{ \pm} x_{2}, \quad \alpha_{0}=f_{3} \alpha_{+}\left(=e_{3} \alpha_{-}\right),
$$

represents the unique highest weight vector of $H^{1,2}\left(G_{A}\right)$, and $\operatorname{Ind}_{L_{0}^{\mathcal{W}}}^{\mathcal{W}}\langle s\rangle \simeq T^{3}$.

Proof. Clearly $e_{3} \alpha_{-}=\alpha_{0}, e_{3} \alpha_{0}=2 \alpha_{+}, e_{3} \alpha_{+}=0$, hence

$$
e_{3} s=\frac{1}{3}\left(\alpha_{0} z_{+}^{2}-\alpha_{0} z_{+}^{2}-2 \alpha_{+} z_{+} z_{-}+2 \alpha_{+} z_{+} z_{-}\right)=0 .
$$

Similarly $f_{3} s=0$ and $s \ell(3) s=0$. Also $Y \cdot s=(-2) s$ and

$$
\nabla s=\hat{\partial}_{1} \zeta_{1}+\hat{\partial}_{2} \zeta_{2}+\hat{\partial}_{3} \zeta_{3}
$$

We see that $\nabla s \in F_{2} M_{A}$. On the other hand $s \in F_{2} M_{A}$, therefore $\nabla[s]=0$ by the definition of the differential of $\operatorname{Gr} M_{A}$. That means $[s]$ is a cycle and it evidently generates a onedimensional subspace $\mathbb{C} s=H^{1,2}\left(G_{A}\right) \simeq \Lambda^{0} \otimes P(0,-2)$ in $H^{1,2}\left(\operatorname{Gr} M_{A}\right)$.

It follows from the lemma that the image of $[s]$ in $H^{1,2}\left(\operatorname{Gr} M_{A}\right)=E^{0}\left(M_{A}\right)^{1,2}$ is the trivial $\mathcal{W}$-sigular vector generating $E^{0}\left(M_{A}\right)^{1,2} \simeq T^{3}$.

Lemma 5.12. Vectors $z_{+}, \zeta_{1} \in M_{A}^{0,1}$ represent the highest weight vectors of $H^{0,1}\left(G_{A}\right)$, and $\left\{z_{ \pm}, \zeta_{i}\right\}$ represent the basis of $H^{0,1}\left(G_{A}\right)$. Also $\operatorname{Ind}_{L_{0}^{\mathcal{W}}}^{\mathcal{W}}\left\langle z_{+}, z_{-}\right\rangle \simeq Q$ and $\operatorname{Ind}_{L_{0}^{\mathcal{W}}}^{\mathcal{W}}\left\langle\zeta_{i}, i=1,2,3\right\rangle \simeq$ $T^{2}$.

This is easy to check, and we leave it to the reader.

Remark 5.13. Considering $E^{0}\left(M_{A}\right)^{0,1}=H^{0,1}\left(M_{A}\right)$ as a $\mathcal{W}$-module, we conclude that $\left[z_{+}\right]$ is a $\mathcal{W}$-singular vector that generates a submodule $Q$ and $\left[\zeta_{1}\right]$ is a $\mathcal{W}$-singular vector that generates a $\mathcal{W}$-submodule isomorphic to $T^{2}$. Of course $z_{+}$also represents an $L$-singular vector of $H^{0,1}\left(M_{A}\right)$.

Let $\mathrm{C}_{e}(u . v . w)=.\sum_{\text {even }} u_{i} v_{j} w_{k}$ and $\mathrm{C}_{o}(u . v . w)=.\sum_{o d d} u_{i} v_{j} w_{k}$ be the sums over all even and odd permutations respectively, where we keep the order of the letters but permute the indices, for example,

$$
\begin{aligned}
\mathrm{C}_{e}\left(d_{.}^{+} d_{.}^{-} d_{.}^{+}\right) & =d_{1}^{+} d_{2}^{-} d_{3}^{+}+d_{2}^{+} d_{3}^{-} d_{1}^{+}+d_{3}^{+} d_{1}^{-} d_{2}^{+}, \\
\mathrm{C}_{e}\left(d_{.}^{+} d_{.}^{-} x .\right) & =d_{1}^{+} d_{2}^{-} x_{3}+d_{2}^{+} d_{3}^{-} x_{1}+d_{3}^{+} d_{1}^{-} x_{2} .
\end{aligned}
$$

By the definition of $\nabla$ one has: $\nabla \mathrm{C}_{e}\left(d_{.^{+}}^{+} d^{-} x.\right) z_{ \pm}=\mathrm{C}_{e}\left(d_{{ }^{+}} d_{\cdot}^{-} d^{ \pm}\right)$and $\nabla \mathrm{C}_{o}\left(d_{\cdot}^{+} d_{.}^{-} x.\right) z_{ \pm}=$ $\mathrm{C}_{o}\left(d^{+} d_{\cdot}^{-} d^{ \pm}\right)$.

Also

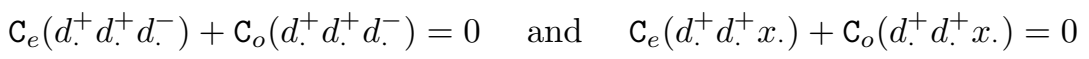

because $\left[d_{i}^{+}, d_{j}^{+}\right]=0$, and similarly for the pair of minuses.

The relation $\left[d_{i}^{+}, d_{j}^{-}\right]+\left[d_{j}^{+}, d_{i}^{-}\right]=0$ implies

$$
\begin{aligned}
& \mathrm{C}_{e}\left(d^{+} d^{-} x .\right)+\mathrm{C}_{o}\left(d_{\cdot}^{-} d^{+} x .\right)+\mathrm{C}_{o}\left(d_{\cdot}^{+} d^{-} x .\right)+\mathrm{C}_{e}\left(d_{\cdot}^{-} d_{\cdot}^{+} x .\right)=0 \\
& \mathrm{C}_{e}\left(d^{+} d^{-} d^{ \pm}\right)+\mathrm{C}_{o}\left(d^{-} d^{+} d^{ \pm}\right)+\mathrm{C}_{o}\left(d^{+} d^{-} d^{ \pm}\right)+\mathrm{C}_{e}\left(d^{-} d^{+} d^{ \pm}\right)=0 \text {, }
\end{aligned}
$$

hence

$$
\mathrm{C}_{o}\left(d_{\cdot}^{+} d_{\cdot}^{-} d_{\cdot}^{+}\right)+\mathrm{C}_{e}\left(d_{\cdot}^{+} d_{\cdot}^{-} d_{\cdot}^{+}\right)=0
$$


One easily checks that

$$
\begin{aligned}
& \mathrm{C}_{e}\left(d_{.^{+}} d_{.}^{+} d_{.}^{-}\right)=-\mathrm{C}_{o}\left(d_{.^{+}}^{+} d_{.}^{-} d_{.^{+}}^{+}\right)-\left(\hat{\partial}_{1} d_{1}^{+}+\hat{\partial}_{2} d_{2}^{+}+\hat{\partial}_{3} d_{3}^{+}\right), \\
& \mathrm{C}_{e}\left(d_{.}^{-} d_{.}^{+} d^{+}\right)=-\mathrm{C}_{o}\left(d_{.}^{+} d_{.}^{-} d^{+}\right)+\left(\hat{\partial}_{1} d_{1}^{+}+\hat{\partial}_{2} d_{2}^{+}+\hat{\partial}_{3} d_{3}^{+}\right) \text {, }
\end{aligned}
$$

hence

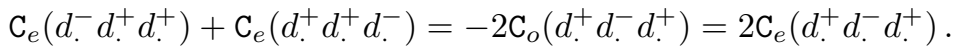

Lemma 5.14. The following elements $t_{ \pm}, \tau_{1}, \tau_{2}, \tau_{3} \in M_{A}^{1,1}$ represent a basis of $H^{1,1}\left(G_{A}\right)$ :

$$
\begin{aligned}
t_{+} & =\mathrm{C}_{e}\left(d_{\cdot}^{+} d_{\cdot}^{+} x .\right) z_{-}-\mathrm{C}_{e}\left(d_{\cdot}^{+} d_{\cdot}^{-} x .\right) z_{+}+\mathrm{C}_{o}\left(d_{\cdot}^{+} d_{\cdot}^{-} x .\right) z_{+}-\mathrm{C}_{o}\left(d_{\cdot}^{-} d_{\cdot}^{+} x .\right) z_{+}, \\
t_{-} & =-\mathrm{C}_{e}\left(d_{\cdot}^{-} d_{\cdot}^{-} x .\right) z_{+}+\mathrm{C}_{e}\left(d_{\cdot}^{-} d_{\cdot}^{+} x .\right) z_{-}-\mathrm{C}_{o}\left(d_{\cdot}^{-} d_{\cdot}^{+} x .\right) z_{-}+\mathrm{C}_{o}\left(d_{\cdot}^{+} d_{\cdot}^{-} x .\right) z_{-}, \\
\tau_{1} & =d_{2}^{+} x_{3} z_{-}+d_{3}^{-} x_{2} z_{+}, \\
\tau_{2} & =d_{3}^{+} x_{1} z_{-}+d_{1}^{-} x_{3} z_{+}, \\
\tau_{3} & =d_{1}^{+} x_{2} z_{-}+d_{2}^{-} x_{1} z_{+} .
\end{aligned}
$$

Here we have isomorphisms $\quad \operatorname{Ind}_{L_{0}^{\mathcal{w}}}^{\mathcal{W}}\left\langle t_{+}, t_{-}\right\rangle \simeq Q, \quad \operatorname{Ind}_{L_{0}^{\mathcal{w}}}^{\mathcal{W}}\left\langle\tau_{i}, i=1,2,3\right\rangle \simeq T^{1}$.

This is also not difficult to prove. Theorem 4.10 shows us the weight subspaces where the representatives should be and we only have to compute the cycles and boundaries in these subspaces. We leave it to the reader to fill in the details.

Remark 5.15. The elements $\left[t_{+}\right],\left[\tau_{3}\right]$ of $E^{0}\left(M_{A}\right)^{1,1}=H^{1,1}\left(M_{A}\right)$ are $\mathcal{W}$-singular vectors, the first one generates the $\mathcal{W}$-submodule isomorphic to $Q$, the second generates the submodule isomorphic to $T^{2}$. At the same time $t_{+}$represents a singular vector of $H^{1,1}\left(M_{A}\right)$, which is not a singular but a secondary singular vector of $M_{A}^{1,1} \simeq M\left(1,0 ; 1 ; y_{A}\right)$.

Proof of Proposition 5.10. We are ready now for the calculating of the differential $\nabla^{(0)}: E^{0}\left(M_{A}\right)^{1,2} \rightarrow E^{0}\left(M_{A}\right)^{0,1}$. ¿From (5.6) it follows that

$$
\nabla^{(0)}[s]=\hat{\partial}_{1}\left[\zeta_{1}\right]+\hat{\partial}_{2}\left[\zeta_{2}\right]+\hat{\partial}_{3}\left[\zeta_{3}\right] .
$$

Using Lemmae 5.11, 5.12 and comparing with (5.5) we see immediately that

$$
\operatorname{Ker}\left(\nabla^{(0)} \mid E^{0}\left(M_{A B}\right)^{1,2}\right)=0 \quad \text { and } \quad \operatorname{Coker}\left(\nabla^{(0)} \mid E^{0}\left(M_{A B}\right)^{0,1}\right)=Q \oplus R .
$$

This proves (2).

For the differential $\nabla^{(0)}: E^{0}\left(M_{A}\right)^{1,1} \rightarrow E^{0}\left(M_{A}\right)^{0,0}$ we need to know $\nabla t_{ \pm}, \nabla \tau_{i}$. Clearly

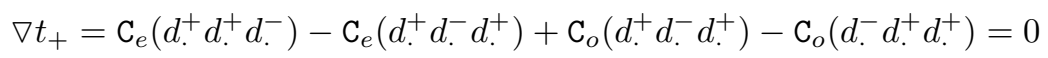

because of (5.8) and (5.11). Now $\nabla t_{-}=\nabla\left(f_{3} t_{+}\right)=f_{3}\left(\nabla t_{+}\right)=0$. And $\nabla \tau_{3}=\left[d_{1}^{+}, d_{2}^{-}\right]=-\hat{\partial}_{3}$. Similarly $\nabla \tau_{i}=-\hat{\partial}_{i}$ for the other values of $i$.

Keeping in mind that $E^{0}\left(M_{A}\right)^{0,0}=H^{0,0}\left(M_{A}\right)=T^{0}$ and Lemma 5.14 we conclude that

$$
\operatorname{Ker}\left(\nabla^{(0)} \mid E^{0}\left(M_{A}\right)^{1,1}\right)=Q \oplus R \quad \text { and } \quad \operatorname{Coker}\left(\nabla^{(0)} \mid E^{0}\left(M_{A}\right)^{0,0}\right)=\mathbb{C},
$$

which gives us (1).

It is clear that $\nabla^{(i)}: E^{i}\left(M_{A}\right)^{1,2} \rightarrow E^{i}\left(M_{A}\right)^{0,1}$ is zero for any $i \geq 1$. Also we see that if any of the differentials $\nabla^{(i)}: E^{i}\left(M_{A}\right)^{1,1} \rightarrow E^{i}\left(M_{A}\right)^{0,0}$ happens to be not zero then $E^{\infty}\left(M_{A}\right)^{0,0}=0$ but this is impossible because $\operatorname{Im}\left(\nabla \mid M_{A}^{0,0}\right) \neq M_{A}^{0,0}$. This proves (3). 
Remark 5.16. Proposition 5.10 shows that $\mathcal{W}$-modules $E^{\infty}\left(M_{A}\right)^{0,1}$ and $E^{\infty}\left(M_{A}\right)^{1,1}$ are isomorphic. We would like to have such an isomorphism $\varphi: H^{0,1}\left(M_{A}\right) \rightarrow H^{1,1}\left(M_{A}\right)$ that would be not only an isomorphim of $\mathcal{W}$-modules but also a morphism (hence isomorphism) of $L$-modules.

Let us notice that the elements

$$
\begin{aligned}
& \theta_{1}=\hat{\partial}_{3} \tau_{2}-\hat{\partial}_{2} \tau_{3}, \\
& \theta_{2}=\hat{\partial}_{1} \tau_{3}-\hat{\partial}_{3} \tau_{1}, \\
& \theta_{3}=\hat{\partial}_{2} \tau_{1}-\hat{\partial}_{1} \tau_{2}
\end{aligned}
$$

represent a basis for the subspace of trivial singular vectors in the $\mathcal{W}$-submodule

$$
\operatorname{Ker} \nabla^{(0)} \cap\left(U(\mathcal{W})\left\langle\left[\tau_{i}\right]\right\rangle\right) \subset E^{0}\left(M_{A}\right)^{1,1},
$$

which is isomorphic to $R$. Also the elements $\zeta_{i}$ represent the basis of the subspace of trivial singular vectors of a $\mathcal{W}$-submodule $\operatorname{Coker}\left(\nabla^{(0)} \mid E^{0}\left(M_{A}\right)^{0,1}\right)$, which is isomorphic to $R$ as well. A similar pair of bases of singular vectors for $\mathcal{W}$-modules which are isomorphic to $Q$ is $\left[t_{ \pm}\right]$and $\left[z_{ \pm}\right]$.

It holds in $H^{0,1}\left(M_{A}\right)$ that $d_{1}^{-}\left[z_{+}\right]-d_{1}^{+}\left[z_{-}\right]=\left[\zeta_{1}\right]$. On the other hand it holds "in $M_{A}^{1,1}$ modulo boundaries" that

$$
d_{1}^{-}\left[t_{+}\right]-d_{1}^{+}\left[t_{-}\right] \equiv 4\left(\hat{\partial}_{3}\left[\tau_{2}\right]-\hat{\partial}_{2}\left[\tau_{3}\right]\right) \equiv 4\left[\theta_{1}\right]
$$

(which could be obtained by straightforward but unfortunately hard computation). Thus if we define a $\mathcal{W}$-isomorphism $\varphi: H^{0,1}\left(M_{A}\right) \rightarrow H^{1,1}\left(M_{A}\right)$ by conditions

$$
\begin{aligned}
& \varphi\left(\left[z_{ \pm}\right]\right)=\frac{1}{4}\left[t_{ \pm}\right], \\
& \varphi\left(\left[\zeta_{i}\right]\right)=\left[\theta_{i}\right],
\end{aligned}
$$

we get a map which is $L_{-}$-linear. As it also maps a singular vector $\left[z_{+}\right]$to a singular vector $\frac{1}{4}\left[t_{+}\right]$, so it is a $L$-homomorphism by the arguments of Remark 2.4(b). Clearly we got the needed isomorphism of the $E(3,6)$-modules.

Proposition 5.17. There are the following isomorphisms of $\mathcal{W}$-modules:

(1) $E^{1}\left(M_{D}\right)^{-1,-2} \simeq \mathbb{C}, \quad E^{1}\left(M_{D}\right)^{0,-1} \simeq R \oplus Q$,

(2) $E^{1}\left(M_{D}\right)^{-1,-1} \simeq R \oplus Q, \quad E^{1}\left(M_{D}\right)^{0,0} \simeq 0$.

(3) The spectral sequence $\left\{E_{i}\left(M_{D}\right)\right\}$ degenerates at $E^{1}$, that is $E^{1}\left(M_{D}\right)^{m, n} \simeq E^{\infty}\left(M_{D}\right)^{m, n}$.

In order to prove this proposition, we shall use representatives for the bases of $H^{m, n}\left(G_{D}\right)$ and calculate the differentials $\nabla^{(i)}$ but first we have to introduce some notations and prove several lemmas.

Consider the associative algebra $U\left(L_{-}\right) \otimes_{\mathbb{C}} V_{D} \simeq M_{D}$. Allowing ourselves a slight abuse of notations we denote $\hat{\partial}_{i}:=\partial_{i} \otimes 1, d_{i}^{ \pm}:=d_{i}^{ \pm} \otimes 1$, and $\partial_{i}:=1 \otimes \partial_{i}$. Furthermore we consider $\Delta^{ \pm}=d_{1}^{ \pm} \partial_{1}+d_{2}^{ \pm} \partial_{2}+d_{3}^{ \pm} \partial_{3}$ and define

$$
\widehat{\Delta}^{ \pm}=\hat{\partial}_{1} d_{1}^{ \pm}+\hat{\partial}_{2} d_{2}^{ \pm}+\hat{\partial}_{3} d_{3}^{ \pm} .
$$

The following two lemmae are straightforward to check.(For odd elements $x, y$ from $M_{D}$ we mean $[x, y]=x y+y x$ as usual.) 
Lemma 5.18. $\quad\left[d_{i}^{ \pm}, \Delta^{ \pm}\right]=0$,

$$
\begin{aligned}
& {\left[d_{1}^{+}, \Delta^{-}\right]=-\left[d_{1}^{-}, \Delta^{+}\right]=\hat{\partial}_{2} \partial_{3}-\hat{\partial}_{3} \partial_{2},} \\
& {\left[d_{2}^{+}, \Delta^{-}\right]=-\left[d_{2}^{-}, \Delta^{+}\right]=\hat{\partial}_{3} \partial_{1}-\hat{\partial}_{1} \partial_{3},} \\
& {\left[d_{3}^{+}, \Delta^{-}\right]=-\left[d_{3}^{-}, \Delta^{+}\right]=\hat{\partial}_{1} \partial_{2}-\hat{\partial}_{2} \partial_{1} .}
\end{aligned}
$$

Lemma 5.19. $\left[d_{i}^{ \pm}, \widehat{\Delta}^{\varepsilon}\right]=0$ for $\varepsilon=-$ or + .

It follows from these two lemmae that, whetever $\varepsilon$, one has

$$
\left[\Delta^{ \pm}, \widehat{\Delta}^{\varepsilon}\right]=0, \quad\left[\widehat{\Delta}^{ \pm}, \widehat{\Delta}^{\varepsilon}\right]=0, \quad\left[\Delta^{ \pm}, \Delta^{\varepsilon}\right]=0 .
$$

Let us supplement the notations introduced in (2.14), (2.15) defining $d:=d_{1}^{-} d_{2}^{-} d_{3}^{-}$. Clearly

$$
\begin{array}{llll}
f_{3} a=b, & f_{3} b=2 c, & f_{3} c=3 d, & f_{3} d=0, \\
e_{3} d=c, & e_{3} c=2 b, & e_{3} b=3 a, & e_{3} a=0 .
\end{array}
$$

It follows from Lemma 5.19 that

$$
\left[a, \widehat{\Delta}^{ \pm}\right]=0, \quad\left[b, \widehat{\Delta}^{ \pm}\right]=0, \quad\left[c, \widehat{\Delta}^{ \pm}\right]=0, \quad\left[d, \widehat{\Delta}^{ \pm}\right]=0 .
$$

\section{Lemma 5.20.}

(1) $\left[a, \Delta^{-}\right]=\widehat{\Delta}^{+} \Delta^{+}=-\Delta^{+} \widehat{\Delta}^{+}$, $\left[b, \Delta^{-}\right]=\widehat{\Delta}^{+} \Delta^{-}+\widehat{\Delta}^{-} \Delta^{+}=\widehat{\Delta}^{+} \Delta^{-}-\Delta^{+} \widehat{\Delta}^{-}$, $\left[c, \Delta^{-}\right]=\widehat{\Delta}^{-} \Delta^{-}=-\Delta^{-} \widehat{\Delta}^{-}$,

$$
d \Delta^{-}=0, \quad \Delta^{-} d=0,
$$

(2) $a \Delta^{+}=0, \quad \Delta^{+} a=0$, $\left[b, \Delta^{+}\right]=\Delta^{+} \widehat{\Delta}^{+}=-\widehat{\Delta}^{+} \Delta^{+}$, $\left[c, \Delta^{+}\right]=\Delta^{+} \widehat{\Delta}^{-}+\Delta^{-} \widehat{\Delta}^{+}=\Delta^{+} \widehat{\Delta}^{-}-\widehat{\Delta}^{+} \Delta^{-}$, $\left[d, \Delta^{+}\right]=\Delta^{-} \widehat{\Delta}^{-}=-\widehat{\Delta}^{-} \Delta^{-}$,

(3) $0=\Delta^{+} a=\Delta^{-} a+\Delta^{+} b=\Delta^{-} b+\Delta^{+} c=\Delta^{-} c+\Delta^{+} d=\Delta^{-} d$, $0=a \Delta^{+}=a \Delta^{-}+b \Delta^{+}=b \Delta^{-}+c \Delta^{+}=c \Delta^{-}+d \Delta^{+}=d \Delta^{-}$, and exactly the same for $\widehat{\Delta}^{ \pm}$(with a hat).

Proof. We can check that

$$
\left[a, d_{i}^{-}\right]=\widehat{\Delta}^{+} d_{i}^{+}, \quad\left[d, d_{i}^{+}\right]=-\widehat{\Delta}^{-} d_{i}^{-},
$$

and $\left[a, \Delta^{-}\right]=\widehat{\Delta}^{+} \Delta^{+},\left[d, \Delta^{+}\right]=\Delta^{-} \widehat{\Delta}^{-}$, follows. Now we apply $f_{3}$ and get the rest in (1), or apply $e_{3}$ and get the rest in (2).

Similarly $\Delta^{+} a=a \Delta^{+}=0, \widehat{\Delta}^{+} a=a \widehat{\Delta}^{+}=0$, then we apply $f_{3}$ and get (3).

Lemma 5.21. Consider the element

$$
\xi=a \Delta^{-} \partial_{+}^{2}+b \Delta^{-} \partial_{+} \partial_{-}+c \Delta^{-} \partial_{-}^{2} \in M\left(0,1 ; 2 ; y_{D}\right) .
$$

It has the following properties:

(a) $\mathrm{g}_{0} \cdot \xi=0$

(b) $e_{0}^{\prime} \cdot \xi=0$,

(c) $e_{0} \cdot \xi \in \operatorname{Im} \nabla$, but $e_{0} \cdot \xi \neq 0$, and $\nabla \xi=0$,

(d) $\xi$ represents a basis for $H^{-1,-2}\left(G_{C D}\right)$. 
Here $\operatorname{Ind}_{L_{0}^{\mathcal{W}}}^{\mathcal{W}}\langle\xi\rangle \simeq T^{0}$.

Proof. The proof of (a) immediate. We get (b) by a straightforward calculation. To get (c) it is enough to notice that

$$
e_{0} \xi=-2\left(d_{2}^{-} d_{3}^{+}+d_{3}^{+} d_{3}^{-}\right) \nabla \partial_{+}-4 d_{2}^{-} d_{3}^{-} \nabla \partial_{-}=\nabla\left(-2\left(d_{2}^{-} d_{3}^{+}+d_{3}^{+} d_{3}^{-}\right) \partial_{+}-4 d_{2}^{-} d_{3}^{-} \partial_{-}\right),
$$

and that

$$
\nabla \xi=a \Delta^{-} \Delta^{+} \partial_{+}^{3}+\left(a \Delta^{-} \Delta^{-}+b \Delta^{-} \Delta^{+}\right) \partial_{+}^{2} \partial_{-}+\left(b \Delta^{-} \Delta^{-}+c \Delta^{-} \Delta^{+}\right) \partial_{+} \partial_{-}^{2}+c \Delta^{-} \partial_{-}^{3}=0,
$$

as it follows form (5.17) and Lemma $5.20(3)$.

For $(\mathrm{d})$ we are to prove that $\xi \notin \operatorname{Im} \nabla$ so $[\xi] \neq 0$ in $H^{-1,-2}\left(G_{D^{\circ}}\right) \simeq \mathbb{C}$. If $\xi \in \operatorname{Im} \nabla$ then $H^{-1,-2}\left(M_{D}\right)=0$, and this would imply the existence of a non-zero differential

$$
\mathrm{S}\left(\mathrm{g}_{-2}\right) \otimes H^{0,-1}\left(G_{D}\right) \longrightarrow \mathrm{S}\left(\mathrm{g}_{-2}\right) \otimes H^{-1,-2}\left(G_{D}\right)
$$

in the spectral sequence for $H\left(M_{D}\right)$ with its image containing $[\xi]$. This is impossible because $Y$-eigenvalue of $\xi$ is 0 , but all eigenvalues of $Y$ on $\mathrm{S}\left(\mathrm{g}_{-2}\right) \otimes H^{0,-1}\left(G_{D}\right)$ are negative as it follows from the description of the homologies in Theorem 4.10.

Corollary 5.22. $\xi$ is a secondary singular vector of the $E(3,6)$-module $M\left(0,1 ; 2 ; y_{D}\right)$.

\section{Lemma 5.23.}

(1) $a c=c a=a \widehat{\Delta}^{-}=-\widehat{\Delta}^{-} a, a b=b a=0$,

(2) $d b=b d=-d \widehat{\Delta}^{+}=\widehat{\Delta}^{+} d, \quad d c=c d=0$,

(3) $a d+d a=b \widehat{\Delta}^{-}=\widehat{\Delta}^{+} c, 2 a d+b c=d a$.

Proof. It is not difficult to check that $a c=a \widehat{\Delta}^{-}$and $c a=-\widehat{\Delta}^{-} a$. Then

$$
a b=e_{3} a c=e_{3} a \widehat{\Delta}^{-}=a \widehat{\Delta}^{+}=0,
$$

and we get (1). Now let

$$
\lambda=2 a d+c \widehat{\Delta}^{+}=2 a d-b \widehat{\Delta}^{-} .
$$

Then $e_{3} \lambda=2 a c+2 b \widehat{\Delta}^{+}=0$ because of (1) and Lemma 5.20 (3). As the $s \ell(2)$-weight of $\lambda$ is zero, so $f_{3} \lambda=0$, which gives us $2 b d-2 c \widehat{\Delta}^{-}=0$. The latter means $b d=c \widehat{\Delta}^{-}=-d \widehat{\Delta}^{+}$.

At the same time $\lambda=-2 d a+b \widehat{\Delta}^{-}$because $\lambda$ is invariant under the Weyl reflection in $S L(2)$ and this implies the first part of (3). We now get the rest of (2) from $e_{3} \lambda=0$ and the rest of (3) applying $f_{3}$ to $a c=a \widehat{\Delta}^{-}$.

Let

$$
\begin{aligned}
q_{+} & =\frac{1}{3}\left(-2 a \Delta^{-} \partial_{+}-b \Delta^{-} \partial_{-}\right), \\
q_{-} & =\frac{1}{3}\left(c \Delta^{+} \partial_{+}+2 d \Delta^{+} \partial_{-}\right), \\
\kappa_{i}=d_{i}^{-} q_{+}-d_{i}^{+} q_{-} &
\end{aligned}
$$

\section{Lemma 5.24.}

(1) $e_{3} q_{+}=0, e_{0}^{\prime} q_{+}=0, \nabla q_{+}=0, e_{0} q_{+}=\nabla\left(-2\left(d_{2}^{+} d_{3}^{-}+d_{2}^{-} d_{3}^{+}\right)\right) \neq 0$,

(2) vectors $q_{+}, \kappa_{1}$ represent the highest weight vectors of $H^{-1,-1}\left(G_{D}\right)$, and $q_{+}, q_{-}, \kappa_{1}, \kappa_{2}, \kappa_{3}$ represent a basis of $H^{-1,-1}\left(G_{D}\right)$,

(3) $q_{+}$represents the (highest weight) singular vector of $H^{-1,-1}\left(M_{D}\right)$,

(4) $q_{+}$is the secondary (highest weight) singular vector in $M\left(0,1 ; 1 ; y_{D}\right)$.

We have isomorphisms $\operatorname{Ind}_{L_{0}^{\mathcal{w}}}^{\mathcal{W}}\left\langle q_{+}, q_{-}\right\rangle \simeq Q, \quad \operatorname{Ind}_{L_{0}^{\mathcal{w}}}^{\mathcal{W}}\left\langle\kappa_{i}, i=1,2,3\right\rangle \simeq T^{2}$.

We leave it to the reader to check the calculations. The rest follows. 
Lemma 5.25. The element $\lambda=2 a d+c \widehat{\Delta}^{+}=-2 d a+b \widehat{\Delta}^{-} \in M_{D}^{0,0}$ represents a basis for $H^{0,0}\left(G_{D}\right)$, the singular vector of $H^{0,0}\left(M_{D}\right)$, $\operatorname{Ind}_{L_{0}^{\mathcal{W}}}^{\mathcal{W}}\langle\lambda\rangle \simeq T^{3}$, and

$$
\nabla \lambda=\widehat{\Delta}^{-} q_{+}-\widehat{\Delta}^{+} q_{-}=\hat{\partial}_{1} \kappa_{1}+\hat{\partial}_{2} \kappa_{2}+\hat{\partial}_{3} \kappa_{3} .
$$

Proof. Let us start with checking the last statement:

$$
\begin{aligned}
\nabla \lambda & =2 a d \Delta^{+} \partial_{+}+c \widehat{\Delta}^{+} \Delta^{+} \partial_{+}+c \widehat{\Delta}^{+} \Delta^{-} \partial_{-} \\
& =2 a \Delta^{-} \widehat{\Delta}^{-} \partial_{+}-b \widehat{\Delta}^{-} \Delta^{+} \partial_{+}+c \widehat{\Delta}^{+} \Delta^{-} \partial_{-} \\
& =a \Delta^{-} \widehat{\Delta}^{-} \partial_{+}+c \widehat{\Delta}^{+} \Delta^{-} \partial_{-} \\
& =a \Delta^{-} \widehat{\Delta}^{-} \partial_{+}+d \widehat{\Delta}^{+} \Delta^{+} \partial_{-} .
\end{aligned}
$$

On the other hand

$$
\begin{aligned}
\widehat{\Delta}^{-} q_{+}-\widehat{\Delta}^{+} q_{-} & =\frac{1}{3}\left(\widehat{\Delta}^{-}\left(-2 a \Delta^{-} \partial_{+}-b \Delta^{-} \partial_{-}\right)-\widehat{\Delta}^{+}\left(c \Delta^{+} \partial_{+}+2 d \Delta^{+} \partial_{-}\right)\right) \\
& =\frac{1}{3}\left(\left(-2 \widehat{\Delta}^{-} a \Delta^{-}+\widehat{\Delta}^{+} c \Delta^{+}\right) \partial_{+}+\left(-\widehat{\Delta}^{-} b \Delta^{-}+2 d \widehat{\Delta}^{+} \Delta^{+}\right) \partial_{-}\right) \\
& =\frac{1}{3}\left(3 a \widehat{\Delta}^{-} \Delta^{-} \partial_{+}+3 d \widehat{\Delta}^{+} \Delta^{+} \partial_{-}\right) .
\end{aligned}
$$

Hence we get the equality. It implies that $\nabla[\lambda]=0$ for $[\lambda] \in \operatorname{Gr} M_{D}$, so $[\lambda]$ is a cycle. The rest is immediate.

$$
\text { Let } \begin{aligned}
r_{+}=a\left(d \partial_{-}+c \partial_{+}\right), & r_{-}=d\left(b \partial_{-}+a \partial_{+}\right), \quad \text { and } \\
\rho_{1} & =\frac{1}{2}\left(d_{2}^{+} d_{3}^{+} \partial_{-}+d_{2}^{-} d_{3}^{-} \partial_{+}\right), \\
\rho_{2} & =\frac{1}{2}\left(d_{3}^{+} d_{1}^{+} \partial_{-}+d_{3}^{-} d_{3}^{-} \partial_{+}\right), \\
\rho_{3} & =\frac{1}{2}\left(d_{1}^{+} d_{2}^{+} \partial_{-}+d_{1}^{-} d_{2}^{-} \partial_{+}\right) .
\end{aligned}
$$

Lemma 5.26. Elements $r_{ \pm}, \rho_{i} \in M_{D}^{0,-1}$ have the properties:

(1) The vector $r_{+}$coincides (up to a sign) with the non-trivial singular vector of $M\left(0,0 ; 1 ; y_{D}\right)$ defined in (2.18): $r_{+}=-w_{3}$,

(2) vectors $r_{+}, \rho_{1}$ represent the highest weight vectors of $H^{0,-1}\left(G_{D}\right)$, and $r_{+}, r_{-}, \rho_{1}, \rho_{2}, \rho_{3}$ represent the basis of $H^{0,-1}\left(G_{D}\right)$,

(3) $d_{1}^{-} r_{+}-d_{1}^{+} r_{-}=\hat{\partial}_{3} \rho_{2}-\hat{\partial}_{2} \rho_{3}$,

(4) $\nabla \rho_{3} \equiv-\frac{2}{3} \xi$ modulo boundaries in $M_{D}^{-1,-2}$.

Here $\quad \operatorname{Ind}_{L_{0}^{\mathcal{W}}}^{\mathcal{W}}\left\langle r_{+}, r_{-}\right\rangle \simeq Q$ and $\quad \operatorname{Ind}_{L_{0}^{\mathcal{w}}}^{\mathcal{W}}\left\langle\rho_{i}, i=1,2,3\right\rangle \simeq T^{1}$.

Equation (2.18) written in our notations is $w_{3}=\left(d a+\widehat{\Delta}^{-} b\right) \partial_{-}+\widehat{\Delta}^{-} a \partial_{+} \cdot(1)$ now follows from Lemma 5.23. The calculations needed to check the rest of the statements are quite straightforward, we leave them to the reader.

Proof of Proposition 5.17. As we compare equations (5.6) with (5.12) and equations (5.16) with Lemma 5.26(4), we notice that the differential $\nabla^{(0)}$ for $E^{0}\left(M_{D}\right)$ is given by the similar formulae as $\nabla^{(0)}$ for $E^{0}\left(M_{A}\right)$. Thus it is possible to repeat the arguments of the proof of Proposition 5.10 and conclude that the values of homologies are as stated.

\section{Proposition 5.27.}

(1) If $f \in M_{A}^{0,1}$ and $f=f^{\prime} z_{+}+f^{\prime \prime} z_{-}$where $f^{\prime}, f^{\prime \prime} \in U\left(L_{-}\right)$, then $\nabla_{6} f=-\left(f^{\prime} r_{+}+f^{\prime \prime} r_{-}\right)$

(2) $\nabla_{6} \cdot \nabla=0$, 
(3) the morphism $\operatorname{Coker}\left(\nabla \mid M_{A}^{0,1}\right) \rightarrow \operatorname{Ker}\left(\nabla \mid M_{D}^{0,-1}\right)$ defined by $\nabla_{6}$ is an isomorphism.

Proof. Evidently the morphism given by the formula in (1) maps $z_{+}$to $w_{3}$, and commutes with $\mathrm{g}_{0}$ and $L_{-}$, hence it is a morphism of $L$-modules, thus equal to $\nabla_{6}$. To show that $\nabla_{6} \cdot \nabla=0$ it is enough to check that $\nabla_{6}$ annihilates vector $d_{i}^{+} z_{+}$, that generates $\operatorname{Im} \nabla$ over $U(L)$. For it we have

$$
\nabla_{6} d_{i}^{+} z_{+}=d_{i}^{+} r_{+}=d_{i}^{+} a\left(d \partial_{-}+c \partial_{+}\right)=0
$$

because $d_{i}^{+} a=0$. The last statement follows.

Corollary 5.28. $H^{0,1}\left(M_{A}\right) \simeq \operatorname{Ker}\left(\nabla_{6} \mid M_{D}^{0,-1}\right)$ is an irreducible module, isomorphic to $I(0,0 ; 1 ;-1)$.

Proof. The submodule $\operatorname{Ker}\left(\nabla_{6} \mid M_{D}^{0,-1}\right)$ is generated by the singular vector $w_{3}$ and, by Theorem 2.10, is has no other singular vectors, hence (by Proposition 1.3h from [KR1]) it is irreducible. As it is isomorphic to $H^{0,1}\left(M_{A}\right)$, it has to be a quotient of $M(0,0 ; 1 ;-1)$, thus $I(0,0 ; 1 ;-1)$.

Proof of Theorem 5.1. The statement (i) of the theorem follows from Proposition 5.10 and (iii) follows from Proposition 5.17. ¿From Remark 5.16 we know that $H^{0,1}\left(M_{A}\right) \simeq$ $H^{1,1}\left(M_{A}\right)$, and Corollary 5.28 shows that $H^{0,1}\left(M_{A}\right) \simeq I(0,0 ; 1 ;-1)$.

Again as we compare equations (5.15) with Lemma 5.26(3), we see that the arguments of Remark 5.16 can be applied to $M_{D}^{-1,-1}, M_{D}^{0,-1}$ as well. This shows us that $H^{0,-1}\left(M_{D}\right) \simeq$ $H^{-1,-1}\left(M_{D}\right) \simeq H^{0,1}\left(M_{A}\right)$. That is all what we need.

\section{The size of $I(p, q ; r ; y)$}

We define the character of a $E(3,6)$-module $V$ by the usual formula:

$$
\operatorname{ch} V=\operatorname{tr}_{V} t^{-3 Y} \text {. }
$$

If $V$ is a degenerate highest weight module, then $\operatorname{ch} V$ is a Laurent series in $t$, whose coefficients are dimensions of eigenspaces of the operator $-3 Y$. Since, as a $\mathrm{g}_{0}$-module

$$
M(p, q ; r ; y)=\mathrm{S}\left(\mathrm{g}_{-2}\right) \otimes \Lambda\left(\mathrm{g}_{-1}\right) \otimes F(p, q ; r ; y)
$$

and the eigenvalues of $-3 Y$ on $g_{-1}$ and $g_{-2}$ are 1 and 2 respectively, we obtain:

$$
\operatorname{ch} M(p, q ; r ; y)=t^{-3 y} \operatorname{dim} F(p, q ; r ; y) R(t),
$$

where $R(t)=(1+t)^{6} /\left(1-t^{2}\right)^{3}$.

Next, let us compute the characters of the modules $I\left(p, 0 ; r ; y_{A}\right)$. By Corollary 5.2, we have an exact sequence

$$
0 \leftarrow I\left(p, 0 ; r ; \frac{2}{3} p-r\right) \leftarrow M\left(p, 0 ; r ; \frac{2}{3} p-r\right) \leftarrow M\left(p+1,0 ; r+1 ; \frac{2}{3} p-r-\frac{1}{3}\right) \leftarrow \cdots,
$$

unless $p=r=0$ or 1 . Hence, using (6.2), we obtain:

$$
\operatorname{ch} I\left(p, 0 ; r ; \frac{2}{3} p-r\right)=t^{3 r-2 p} R(t) \sum_{j=0}^{\infty}(-1)^{j} \frac{(j+p+2)(j+p+1)(j+r+1)}{2} t^{j}
$$

unless $p=r=0$ or 1 . In order to sum this series for $|t|<1$, we use the identity

$$
\sum_{j=0}^{\infty}(-1)^{j}\left(\begin{array}{c}
j+m \\
m
\end{array}\right) t^{j}=\frac{1}{(1+t)^{m+1}} .
$$


We get (unless $p=r=0$ or 1 ):

$$
\operatorname{ch} I\left(p, 0 ; r ; \frac{2}{3} p-r\right)=t^{3 r-2 p} R(t)\left(\frac{3}{(1+t)^{4}}+\frac{2 p+r-2}{(1+t)^{3}}+\frac{p^{2}+2 p r-p}{2(1+t)^{2}}+\frac{p^{2} r+p r}{2(1+t)}\right) .
$$

In particular, for $r>0$ we have:

$$
\operatorname{ch} I(0,0 ; r ;-r)=t^{3 r} R(t) \frac{(r+1)+(r-2) t}{(1+t)^{4}} .
$$

Next, we compute the characters of the modules $I\left(0, q ; r ; y_{D}\right)$. By Corollary 5.2, we have an exact sequence

$$
0 \rightarrow I\left(0, q ; r ; r-\frac{2}{3} q\right) \rightarrow M\left(0, q+1 ; r+1 ; r-\frac{2}{3} q-\frac{1}{3}\right) \rightarrow \cdots
$$

unless $(q, r)=(0,0),,(1,1),(0,1)$ and $(1,2)$, in the last case the exactness being broken by a 1-dimensional space. Hence, except for these four cases, we have:

$$
\operatorname{ch} I\left(0, q ; r ; r-\frac{2}{3} q\right)=t^{4 q-6 r} \operatorname{ch} I\left(q+1,0 ; r+1 ; r-\frac{2}{3} q-\frac{1}{3}\right) .
$$

One computes similarly the characters of $B$ and $C$ type, but they are more cumbersome and we omit them.

Definition 6.1. Given a $\mathrm{S}\left(\mathrm{g}_{-2}\right)$-module $V$, we define

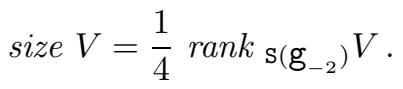

It is clear that size $V$ can be expressed via $\operatorname{ch} V$ as follows:

$$
\text { size } V=\frac{1}{4} \lim _{t \rightarrow-1}\left(1-t^{2}\right)^{3} \operatorname{ch} V .
$$

In particular, we obtain from (6.2):

$$
\text { size } M(p, q ; r ; y)=16 \operatorname{dim} F(p, q ; r ; y) .
$$

One computes immediately the sizes of modules $I\left(p, 0 ; r ; y_{A}\right)$ and $I\left(p, 0 ; r ; y_{D}\right)$ using (6.5), (6.7) and (6.8). The sizes of $I\left(p, 0 ; r ; y_{B}\right)$ and $I\left(p, 0 ; r ; y_{C}\right)$ are then computed by adding a finite number of terms using exact sequences from Figure 3; it follows that in both cases the size is a polynomial of degree $\leq 2$ in $p$ and of degree $\leq 1$ in $r$. The final result of the calculations is given by the following.

Theorem 6.2. (A) If $(p, r) \neq(0,0)$ or $(1,1)$, then

$$
\begin{aligned}
\text { size } I\left(p, 0 ; r ; \frac{2}{3} p-r\right) & =2 r\left(2 p^{2}+4 p+1\right)+\left(2 p^{2}+2 p-1\right) ; \\
\text { size } I\left(1,0 ; 1 ;-\frac{1}{3}\right) & =16 .
\end{aligned}
$$

(B) size $I\left(p, 0 ; r ; \frac{2}{3} p+r+2\right)=2 r\left(2 p^{2}+4 p+1\right)+\left(6 p^{2}+14 p+5\right)$.

(C) size $I\left(0, q ; r ;-\frac{2}{3} q-r-2\right)=2 r\left(2 q^{2}+8 q+7\right)+\left(2 q^{2}+10 q+11\right)$.

(D) If $(q, r) \neq(0,0)$ or $(1,1)$, then

$$
\begin{aligned}
\text { size } I\left(0, q ; r ; r-\frac{2}{3} q\right) & =2 r\left(2 q^{2}+6 q+7\right)+\left(6 q^{2}+22 q+17\right) \\
\text { size } I\left(0,1 ; 1 ; \frac{1}{3}\right) & =74
\end{aligned}
$$

Remark 6.3. It follows from (6.1) and Figure 3 that the sizes of the even and odd parts of all modules $I(p, q ; r ; y)$ are equal. 


\section{The secondary $\mathbb{Z}$-grading of $E(5,10)$ as a $E(3,6)$-module.}

Recall that in Section 1 we defined the secondary $\mathbb{Z}$-grading

$$
E(5,10)=\prod_{j \geq-1} U^{j}
$$

by letting

$$
\operatorname{deg} x_{i}=0=\operatorname{deg} \partial_{i} \text { for } i=1,2,3 ; \operatorname{deg} x_{j}=1=-\operatorname{deg} \partial_{j} \text { for } j=4,5 ; \operatorname{deg} d=-\frac{1}{2},
$$

so that $U^{0}$ is isomorphic to $E(3,6)$, and each $U^{j}$ is a $E(3,6)$-module. Each of the subspaces $U^{j}$ carries a $\mathbb{Z}$-grading by finite-dimensional subspaces induced by the consistent grading of $E(5,10)$ defined in Section 11:

$$
E(5,10)^{j}=\prod_{i \in \mathbb{Z}} U_{i}^{j} .
$$

Each of the $E(3,6)$-modules $U^{j}$ is a linearly compact space, hence the dual modules are discrete spaces:

$$
U^{j *}=\oplus_{j \in \mathbb{Z}} U_{i}^{j *}
$$

Note that all $U_{i}^{j *}\left(\right.$ and $\left.U_{i}^{j}\right)$ are $g_{0}$-modules.

Since $E(5,10)_{i}^{j}=0$ for $i<-2$, it follows that all $E(3,6)$-modules $U^{j *}$ are $L_{0}$-locally finite, hence they are objects from the category $\mathcal{P}\left(L, L_{0}\right)$ considered in [KR1].

Theorem 7.1. (a) The E(3,6)-module $U^{j *}$ has size $2 j+3, j=-1,0,1, \ldots$

(b) One has the following isomorphisms of $E(3,6)$-modules:

$$
\begin{aligned}
U^{-1 *} & \simeq I(0,0 ; 1 ;-1), U^{0 *} \simeq I\left(1,0 ; 0 ; \frac{2}{3}\right), \\
U^{j *} & \simeq I(0,0 ; j-1 ; j+1) \text { for } j \geq 1 .
\end{aligned}
$$

Proof. It is straightforward to check (a) using the construction of $U^{j}$.

It follows from (7.1) that $U^{j *}$ has a maximal submodule $U_{(0)}^{j *}$ such that $U^{j *} / U_{(0)}^{j *}$ is an irreducible module from $\mathcal{P}\left(L, L_{0}\right)$. It is easy to see that the lowest non-zero space in (7.1) is isomorphic to the $\mathrm{g}_{0}$-modules $F(0,0 ; 1 ;-1), F\left(1,0 ; 0 ; \frac{2}{3}\right)$ and $F(0,0 ; j-1 ; j+1)$ for $j=-1, j=0$ and $j \geq 1$, respectively. Hence the $E(3,6)$-module $U^{j *} / U_{(1)}^{j *}$ is isomorphic to $I(0,0 ; 1 ;-1), I\left(1,0 ; 0 ; \frac{2}{3}\right)$ and $(I(0,0 ; j-1 ; j+1))$, respectively. It follows from Theorem 6.2 that these $E(3,6)$-modules have the same size as $U^{j *}$ (given by (a)). Since $U^{j *}$ as $\mathrm{S}\left(\mathrm{g}_{-2}\right)$ modules have no torsion, it follows that $U_{(1)}^{j *}=0$, hence (b).

We conclude this section by an explicit description of the module $U^{-1 *}=I(0,0 ; 1 ;-1)$, which is a (non-trivial) $E(3,6)$ - module of minimal possible size $(=1)$.

Denote by $\Omega_{3}^{k}$ the space of differential $k$-forms over $\mathbb{C}\left[\left[x_{1}, x_{2}, x_{3}\right]\right]$ and by $\Omega_{3 c \ell}^{k}$ the subspace of closed forms. The Lie algebra $W_{3}$ of all formal vector fields acts on $\Omega_{3}^{k}$ via Lie derivative, leaving $\Omega_{3 c \ell}^{k}$ invariant. For $\lambda \in \mathbb{C}$, define a $\lambda$-twisted $W_{3}$-module structure on $\Omega_{3}^{k}$, which we denote by $\left(\Omega_{3}^{k}\right)^{\lambda}$, by letting

$$
D \omega=L_{D} \omega+\lambda(\operatorname{div} D) \omega, \quad D \in W_{3}, \omega \in \Omega_{3}^{k} .
$$

Note, that we have a canonical isomorphism of $W_{3}$-modules:

$$
\left(\Omega_{3}^{3}\right)^{\lambda} \simeq\left(\Omega_{3}^{0}\right)^{1+\lambda} .
$$

Recall the following explicit construction of $E(3,6)$ CK:

$$
E(3,6)_{\overline{0}}=W_{3}+\Omega_{3}^{0} \otimes s \ell(2), \quad E(3,6)_{\overline{1}}=\left(\Omega_{3}^{1}\right)^{-\frac{1}{2}} \otimes \mathbb{C}^{2},
$$


with the obvious bracket on $E(3,6)_{\overline{0}}$ and between $E(3,6)_{\overline{0}}$ and $E(3,6)_{\overline{1}}$, and the following bracket on $E(3,6)_{\overline{1}}$ :

$$
\left[\omega \otimes v, \omega^{\prime} \otimes v^{\prime}\right]=\left(\omega \wedge \omega^{\prime}\right) \otimes\left(v \otimes v^{\prime}\right)+\left(d \omega \wedge \omega^{\prime}+\omega \wedge d \omega^{\prime}\right) \otimes v \cdot v^{\prime},
$$

where $v \cdot v^{\prime} \in S^{2} \mathbb{C}^{2} \simeq s \ell(2), v \wedge v^{\prime} \in \Lambda^{2} \mathbb{C}^{2} \simeq \mathbb{C}$. We have identified here $\Omega_{3}^{0}$ with $\left(\Omega_{3}^{3}\right)^{-1}$ (see $(7.2)$ ), and also use the identification

$$
W_{3} \simeq\left(\Omega_{3}^{2}\right)^{-1} .
$$

(We fix a volume form in $\mathbb{C}^{3}$ when we define the twisted action.)

Proposition 7.2. The $E(3,6)$-module $I=I(0,0 ; 1 ;-1)$ is constructed explicitly as follows:

$$
I_{\overline{0}}=\left(\Omega_{3}^{0}\right)^{\frac{1}{2}} \otimes \mathbb{C}^{2}, \quad I_{\overline{1}}=\Omega_{3 c \ell}^{2} .
$$

$E(3,6)_{\overline{0}}$ acts in the obvious way $\left(\Omega_{3}^{0} \otimes s \ell(2)\right.$ acts trivially on $\left.I_{\overline{1}}\right)$, and $E(3,6)_{\overline{1}}$ acts as follows $\left(\omega_{i} \in \Omega_{3}^{i}, u, v \in \mathbb{C}^{2}\right)$ :

$$
\begin{aligned}
\left(\omega_{1} \otimes u\right)\left(\omega_{0} \otimes v\right) & =d\left(\omega_{0} \omega_{1}\right) \otimes(u \wedge v), \\
\left(\omega_{1} \otimes u\right) \omega_{2} & =\left(\omega_{1} \wedge \omega_{2}\right) \otimes u,
\end{aligned}
$$

where we use identifications (7.3), $u \wedge v \in \Lambda^{2} \mathbb{C}^{2} \simeq \mathbb{C}$ and $\omega_{1} \wedge \omega_{2} \in\left(\Omega_{3}^{3}\right)^{-\frac{1}{2}} \simeq\left(\Omega_{3}^{0}\right)^{\frac{1}{2}}$.

Proof. It is straightforward from definitions.

\section{A relation to the Standard Model.}

Recall that the $\mathrm{g}_{0}$-module $\mathrm{g}_{-1}$ is isomorphic to $F\left(1,0 ; 1 ;-\frac{1}{3}\right)$. The action of the compact form $\mathfrak{k}=s u(3)+s u(2)+i \mathbb{R} Y$ of $\mathrm{g}_{0}$ on $\mathrm{g}_{-1}$ exponentiates to a faithful representation of the compact group

$$
K=(S U(3) \times S U(2) \times U(1)) / C,
$$

where $C$ is a central subgroup of order 6 . Recall that the group $K$ is the group of symmetries of the Standard Model.

It is straightforward to check the following.

Lemma 8.1. The $\mathrm{g}_{0}$-module $F(p, q ; r ; y)$ exponentiates to $K$ iff the following two conditions hold:

$$
\begin{array}{r}
y \in \frac{1}{3} \mathbb{Z}, \\
2(p-q)+3 r-3 y \in 6 \mathbb{Z} .
\end{array}
$$

Since the action of $\mathrm{g}_{0}$ on $\mathrm{g}_{-1}$ and hence on $\mathrm{g}_{-2}$ exponentiates to $K$ (this, in fact, is true for $\mathrm{g}_{1}$ and hence for all $\mathrm{g}_{j}$ as well), we obtain from the isomorphism (1.10) that a $E(3,6)$-module $M(p, q ; r ; y)$ restricted to $\mathrm{g}_{0}$ exponentiates to $K$ iff (8.1) and 8.2) hold. In particular, we obtain the following corollary of Lemma 8.1 and Theorem 1.2 .

Corollary 8.2. All degenerate $E(3,6)$-modules $I\left(p, q ; r ; y_{X}\right)$ exponentiate to $K$.

Definition 8.3. A $K$-module $F(p, q ; r ; y)$ is called a fundamental particle multiplet if the following two properties hold:

when restricted to $S U(3)$, only the 1-dimensional,

the two fundamental and the adjoint representations occur,

$$
\frac{1}{2}|y+h| \leq 1 \text {, where } h \text { is any eigenvalue of } H=\left(\begin{array}{rr}
1 & 0 \\
0 & -1
\end{array}\right) \in i s u(2) .
$$


Condition (8.4) means that modulus of charges (given by Gell-Mann-Nishijima formula) of all particles in the multiplet do not exceed 1.

It is immediate to see that all $K$-modules $F(p, q ; r ; y)$ for which (8.3) and (8.4) hold are listed in the left hand side of Table 1 . The right half contains all the fundamental particles of the Standard Model: the upper part consists of three generations of quarks and the middle part of three generations of leptons (these are all fundamental fermions from which the matter is built), and the lower part consists of the fundamental bosons (which mediate the strong and electroweak interactions).

TABLE 1.

\begin{tabular}{|c|c|c|c|c|}
\hline multiplets & charges & & particles & \\
\hline$(01,1,1 / 3)$ & $2 / 3,-1 / 3$ & $\left(\begin{array}{l}u_{L} \\
d_{L}\end{array}\right)$ & $\left(\begin{array}{l}c_{L} \\
s_{L}\end{array}\right)$ & $\left(\begin{array}{l}t_{L} \\
b_{L}\end{array}\right)$ \\
\hline$(10,1,-1 / 3)$ & $-2 / 3,1 / 3$ & $\left(\begin{array}{l}\widetilde{u}_{R} \\
\widetilde{d}_{R}\end{array}\right)$ & $\left(\begin{array}{l}\widetilde{c}_{R} \\
\widetilde{s}_{R}\end{array}\right)$ & $\left(\begin{array}{c}\widetilde{t}_{R} \\
\widetilde{b}_{R}\end{array}\right)$ \\
\hline$(10,0,-4 / 3)$ & $-2 / 3$ & $\widetilde{u}_{L}$ & $\widetilde{c}_{L}$ & $\tilde{t}_{R}$ \\
\hline$(01,0,4 / 3)$ & $2 / 3$ & $u_{R}$ & $c_{R}$ & $t_{R}$ \\
\hline$(01,0,-2 / 3)$ & $-1 / 3$ & $d_{R}$ & $s_{R}$ & $b_{R}$ \\
\hline$(10,0,2 / 3)$ & $1 / 3$ & $\tilde{d}_{L}$ & $\widetilde{s}_{L}$ & $\widetilde{b}_{L}$ \\
\hline---- & --- & ---- & ---- & - \\
\hline$(00,1,-1)$ & $0,-1$ & $\left(\begin{array}{l}\nu_{L} \\
e_{L}\end{array}\right)$ & $\left(\begin{array}{c}\nu_{\mu L} \\
\mu_{L}\end{array}\right)$ & $\left(\begin{array}{c}\nu_{\tau L} \\
\tau_{L}\end{array}\right)$ \\
\hline$(00,1,1)$ & 0,1 & $\left(\begin{array}{c}\widetilde{\nu}_{R} \\
\widetilde{e}_{R}\end{array}\right)$ & $\left(\begin{array}{c}\widetilde{\nu}_{\mu R} \\
\tilde{\mu}_{R}\end{array}\right)$ & $\left(\begin{array}{c}\widetilde{\nu}_{\tau R} \\
\widetilde{\tau}_{R}\end{array}\right)$ \\
\hline$(00,0,2)$ & 1 & $\widetilde{e}_{L}$ & $\widetilde{\mu}_{L}$ & $\widetilde{\tau}_{L}$ \\
\hline$(00,0,-2)$ & -1 & $e_{R}$ & $\mu_{R}$ & $\tau_{R}$ \\
\hline$(11,0,0)$ & 0 & gluons & & \\
\hline$(00,2,0)$ & $1,-1,0$ & $W^{+}, W^{-}, Z$ & (gauge bosons) & \\
\hline$(00,0,0)$ & 0 & $\gamma$ & (photon) & \\
\hline$(11,0, \pm 2)$ & \pm 1 & - & & \\
\hline
\end{tabular}

One can show that the direct sum of degenerate $E(3,6)$-modules

$$
I(0,0 ; 1 ;-1) \oplus I\left(1,0 ; 0 ; \frac{2}{3}\right) \oplus I(0,0 ; 0 ; 2) \oplus I(0,0 ; 0 ;-2)
$$

contains all the fundamental particle multiplets once, except for $\left(01,1, \frac{1}{3}\right)$ which is contained twice.

\section{APPENDIX: A spectral sequence for a filtered module with a differential that does not preserve the filtration.}

Usually a spectral sequence is constructed for a differential filtered module but we relax the conditions and suppose that there is a module with a differential and a filtration where the differential does not preserve the filtration but only the condition (9.1) below is true. We show that the construction still works with minor alterations. 
Let $A$ be a module with a filtration (we follow more or less the notations of [M, Ch. XI, Section 3]):

$$
\cdots \subset F_{p-1} A \subset F_{p} A \subset F_{p+1} A \subset \cdots \quad(p \in \mathbb{Z})
$$

and with a differential $d: A \rightarrow A, d^{2}=0$, such that

$$
d\left(F_{p} A\right) \subset F_{p-s+1} A
$$

for some fixed $s$ and every $p \in \mathbb{Z}$. The usual case of differential module corresponds to $s=1$, but for our main application $s=0$.

We claim that there is a spectral sequence $E=\left\{E^{r}, d^{r}\right\}_{r \in \mathbb{Z}}$, which, as usual, is a sequence of $\mathbb{Z}$-graded modules $E^{r}=\sum_{p \in \mathbb{Z}} E_{p}^{r}$, each with a differential

$$
d^{r}: E_{p}^{r} \rightarrow E_{p-r}^{r}
$$

and with isomorphisms

$$
H\left(E^{r}, d^{r}\right) \simeq E^{r+1},
$$

and for this spectral sequence there are natural isomorphisms:

$$
E_{p}^{r} \simeq F_{p} A / F_{p-1} A \text { for } r \leq s-1 \text { and } d^{r}=0 \text { if } r<s-1, d^{s-1}=\operatorname{gr} d,
$$

hence

$$
E_{p}^{s} \simeq H\left(F_{p} A / F_{p-1} A\right) .
$$

In other words, $E^{s}$ is isomorphic to the homology of the module $\mathrm{Gr} A$ with respect to the induced differential

$$
\text { gr } d: F_{p} A / F_{p-1} A \rightarrow F_{p-s+1} A / F_{p-s} A .
$$

Let us mention that if $A$ is a graded filtered module with a grading of some kind (bigrading, etc.) then the $E^{r}$ inherit similar gradings.

To construct the spectral sequence we keep on with the usual construction of the spectral sequence for a differential module.

Introduce submodules

$$
Z_{p}^{r}=\left\{a \mid a \in F_{p} A, d a \in F_{p-r} A\right\},
$$

define subquotients

$$
E_{p}^{r}=\left(Z_{p}^{r}+F_{p-1} A\right) /\left(d Z_{p+r-1}^{r-1}+F_{p-1} A\right),
$$

and differentials $d^{r}: E_{p}^{r} \rightarrow E_{p-r}^{r}$ as the homomorphisms induced on the subquotients by the differential $d$ of $A$.

In the case $r \leq s-1$ one has: $Z_{p}^{r}=F_{p} A$, and clearly

$$
d Z_{p+r-1}^{r}=d\left(F_{p+r-1} A\right) \subset F_{p+r-s} A \subset F_{p-1} A .
$$

Therefore

$$
E_{p}^{r}=F_{p} A / F_{p-1} A \text { for } r \leq s-1 .
$$

On the other hand (9.8) shows that

$$
d Z_{p+r}^{r} \subset F_{p+1+r-s} A \text { if } r<s-1 .
$$

Hence

$$
d Z_{p+r}^{r} \subset F_{p-1} A \text { and so } d^{r} \equiv 0 \text { for } r<s-1 .
$$


Thus $E^{s-1}$ coincides with Gr $A$ and $d^{s-1}$ coincides with gr $d$ and (9.2) for $r \leq s-1$ implies (9.3) and (9.4).

To prove (9.2) we notice first that $Z_{p}^{r} \cap F_{p-1} A=Z_{p-1}^{r-1}$, hence (9.7) implies

$$
E_{p}^{r} \simeq Z_{p}^{r} /\left(d Z_{p+r-1}^{r-1}+Z_{p-1}^{r-1}\right) .
$$

Here we use the standard module isomorphism:

$$
(U+W) /(V+W) \simeq U /(V+U \cap W)
$$

for submodules $U, V, W$ of a module $A$ such that $U \supset V$.

We write in the same way

$$
E_{p-r}^{r} \simeq Z_{p-r}^{r} /\left(d Z_{p-1}^{r-1}+Z_{p-r-1}^{r-1}\right) .
$$

But

$$
\left\{x \mid x \in Z_{p}^{r}, d x \in Z_{p-r-1}^{r-1}\right\}=\left\{x \mid x \in Z_{p}^{r}, d x \in F_{p-r-1} A\right\}=Z_{p}^{r+1} .
$$

This forces us to conclude that $\operatorname{ker}\left(d^{r}: E_{p}^{r} \rightarrow E_{p-r}^{r}\right)$ coincides with the image in $E_{p}^{r}$ of $Z_{p}^{r+1} \subset Z_{p}^{r}$

Thus, taking into account that $Z_{p}^{r+1} \supset d Z_{p+r-1}^{r-1}$ and that $Z_{p}^{r+1} \cap Z_{p-1}^{r-1}=Z_{p-1}^{r}$, we use (9.9) to establish the following isomorphism:

$$
\operatorname{ker}\left(d^{r}: E_{p}^{r} \rightarrow E_{p-r}^{r}\right) \simeq Z_{p}^{r+1} /\left(d Z_{p+r-1}^{r-1}+Z_{p-1}^{r}\right) .
$$

Now, because of (9.9),

$$
E_{p+r}^{r} \simeq Z_{p+r}^{r} /\left(d Z_{p+2 r-1}^{r-1}+Z_{p+r-1}^{r-1}\right)
$$

hence, as $d Z_{p+r}^{r} \subset Z_{p}^{r}$, we see that

$$
\operatorname{Im}\left(d^{r}: E_{p+r}^{r} \rightarrow E_{p}^{r}\right) \simeq\left(d Z_{p+r}^{r}+Z_{p-1}^{r}\right) /\left(d Z_{p+r-1}^{r-1}+Z_{p-1}^{r}\right) .
$$

This, together with 9.10)

$$
H\left(E_{p}^{r}\right) \simeq Z_{p}^{r+1} /\left(d Z_{p+r}^{r}+Z_{p-1}^{r}\right) \simeq E_{p}^{r+1} .
$$

So (9.3) is established.

Quite similar to the filtered differential module situation ([M, Ch. XI, Prop. 3.2]) we get the convergence of the spectral sequence under additional conditions on the filtration.

Proposition 9.1. If $\cup_{p} F_{p} A=A$ and for some $N, F_{-N} A=0$, then the spectral sequence converges.

The latter means that for every $p$ and large enough $r$ we get a commutative diagram of natural morphisms

$$
\begin{array}{rlrl}
E_{p}^{r} & \rightarrow & E_{p}^{r+1} & \rightarrow \cdots \\
& \searrow & \searrow \downarrow \\
& & F_{p}(H(A)) / F_{p-1}(H(A))=\operatorname{Gr}_{p}(H(A))
\end{array}
$$

that identifies $\operatorname{Gr}_{p}(H(A)) \simeq \underset{r}{\lim _{\longrightarrow}} E_{p}^{r}$, or $\operatorname{Gr}(H(A)) \simeq E_{p}^{\infty}$.

The morphisms

$$
E_{p}^{r} \rightarrow E_{p}^{r+1} \rightarrow \cdots
$$

are defined because $Z_{-N}^{r}=0$ so $E_{-N}^{r}=0$, therefore $\operatorname{ker}\left(d^{r}: E_{p}^{r} \rightarrow E_{p-r}^{r}\right)=E_{p}^{r}$ for given $p$ and $r$ large enough. 
Moreover $E_{p}^{r}=F_{p} A /\left(d Z_{p+r-1}^{r-1}+F_{p-1} A\right)$ and $E_{p}^{r} \rightarrow E_{p}^{r+1}$ are surjective for large $r$. Also for large $r, Z_{p}^{r}=(\operatorname{ker} d) \cap F_{p} A$ and then (9.7) shows that the inductive limit $E_{p}^{\infty}$ of the system $\left\{E_{p}^{r} \rightarrow E_{p}^{r+1} \rightarrow \cdots\right\}$ is

$$
E_{p}^{\infty}=\underset{r}{\lim _{\longrightarrow}} E_{p}^{r}=\left((\operatorname{ker} d) \cap F_{p} A+F_{p-1} A\right) /\left(\cup_{r} d Z_{p+r-1}^{r-1}+F_{p-1} A\right) .
$$

But $\cup_{r} d Z_{p+r-1}^{r-1}=(d A) \cap F_{p} A$, hence

$$
E_{p}^{\infty} \simeq\left((\operatorname{ker} d) \cap F_{p} A+F_{p-1} A\right) /\left(d A \cap F_{p} A+F_{p-1} A\right)=\operatorname{Gr}_{p}(H(A)),
$$

as stated above.

\section{References}

[CK] Cheng, S-J., Kac, V.: Structure of some Z-graded Lie superalgebras of vector fields. Transformation Groups, 4 (1999), 219-272.

[K] Kac, V.: Classification of infinite-dimensional simple linearly compact Lie superalgebras. Advances in Math. 139 (1998), 1-55.

[KR1] Kac, V., Rudakov, A.: Representations of the exceptional Lie superalgebra E(3,6) I: Degeneracy conditions, ESI preprint no 921, 2000.

[KR2] Kac, V., Rudakov, A.: Representations of the exceptional Lie superalgebra E(3,6) III: Classification of singular vectors, in preparation.

[M] Mac Lane, S.: Homology (3-rd corr.printing). Springer-Verlag: NY-Heidelberg-Berlin, 1975.

[R] Rudakov, A.: Irreducible representations of infinite-dimensional Lie algebras of Cartan type. Math.USSR-Izvestia 8 (1974), 836-866.

\section{Authors' addresses:}

Department of Mathematics, MIT, Cambridge MA 02139, USA

email: kac@math.mit.edu

Department of Mathematics, NTNU, Gløshaugen, N-7491 Trondheim, Norway

email: rudakov@math.ntnu.no 